\title{
Chemical Synthesis of Multifunctional Air Pollutants: Terpene-derived Nitrooxy Organosulfates
}

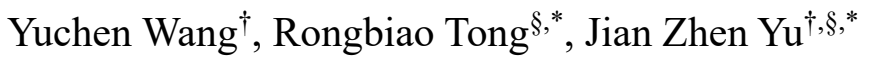 \\ ${ }^{\dagger}$ Division of Environment \& Sustainability, ${ }^{\S}$ Department of Chemistry, Hong Kong University \\ of Science \& Technology, Clear Water bay, Kowloon, Hong Kong, China
}

*Corresponding Authors

Department of Chemistry

Hong Kong University of Science \& technology

Clear Water bay, Kowloon, Hong Kong

Phone: (852) 2358 7389, Fax: (852) 2358 1594, Email: rtong@ust.hk, jian.yu@ust.hk

This supplemental information contains 1 table, 24 figures, and 1 scheme, totaling 53 pages including the cover page. 


\section{Table of Content}

S1. Formation scheme of NOSs in the atmosphere and summary information of synthesized NOSs in this work

Scheme S1. General understanding of formation of NOS in atmosphere

Table S1. Summary information of nitrooxy organosulfates synthesized in this study

\begin{tabular}{|lc|}
\hline S2. Experimental Section & $\underline{\mathrm{S}-4}$ \\
\hline S2.1 General & $\underline{\mathrm{S}-4}$ \\
\hline S2.2 General procedure for the preparation of terpene oxide & $\underline{\mathrm{S}-4}$ \\
\hline S2.3 General procedure for the preparation of Bromohydrins & $\underline{\mathrm{S}-5}$ \\
\hline S2.4 General procedure for the preparation of hydroxynitrate & $\underline{\mathrm{S}-6}$ \\
\hline S2 5 Procedure for the preparation of nitrooxy organosulfate sodium salt & $\underline{\mathrm{S}-8}$ \\
\hline
\end{tabular}

S2.5 Procedure for the preparation of nitrooxy organosulfate sodium salt $\quad \underline{\mathrm{S}-8}$

S2.6 Purity of compounds 4a' and 5b' $\underline{\text { S-10 }}$

S3. ${ }^{1} \mathrm{H}$ and ${ }^{13} \mathrm{C}$ NMR Spectra (S-12 to S-47) $\quad \underline{\mathrm{S}-12}$

Figure S1. NMR spectra of compound 2a

Figure S2. NMR spectra of compound 2b

Figure S3. NMR spectra of compound 2c

Figure S4. NMR spectra of compound $\mathbf{6 b}$

Figure S5. NMR spectra of compound $\mathbf{6 d}$

Figure S6. NMR spectra of compound $\mathbf{3 b}$ '

Figure S7. NMR spectra of compound 3d

Figure S8. NMR spectra of regio-isomers $\mathbf{3 b} \& \mathbf{3} \mathbf{3}$ '

Figure S9. NMR spectra of mixture 3a' \& Carveol

Figure S10. NMR spectra of compound 3a

Figure S11. NMR spectra of compound Campholenaldehyde

Figure S12. NMR spectra of compound 3c

Figure S13. NMR spectra of compound $\mathbf{4} \mathbf{b}^{\prime}=\mathbf{2}^{\mathbf{0}}{ }_{-} \mathbf{L m N O S}$

Figure S14. NMR spectra of regio-isomers $4 \mathrm{~b}^{\prime}=\mathbf{2}^{\mathbf{0}}{ }_{-} \mathbf{L m N O S} \& \mathbf{4 b}=\mathbf{3}^{\mathbf{0}}$ LmNOS

Figure S15. NMR spectra of compound $\mathbf{4 a} \mathbf{a}^{\prime}=\mathbf{2}^{\mathbf{0}}{ }_{-}$ApNOS

Figure S16. NMR spectra of compound $4 \mathbf{c}=\mathbf{2}^{\mathbf{0}}{ }_{-}$BcpNOS

Figure S17. NMR spectra of compound $5 \mathbf{b}^{\prime}=\mathbf{2}^{\circ} \mathbf{L k N O S}$

S4. Hydrolysis of $\mathbf{3 a}$ in $\mathrm{CD}_{3} \mathrm{OD}$

Figure S18. First order kinetic analysis for hydrolysis of $3 \mathrm{a}$ in $\mathrm{CD}_{3} \mathrm{OD}$

S5. MS/MS data collected from ambient samples compared to authentic standards

Figure S19. MS/MS spectra of NOS $_{\mathrm{TP}}$ compounds in ambient samples and in standard solutions.

S6. Additional supplementary plots

Figure S20. Correlation plots of MW295 peaks in ambient samples

Figure S21. Comparison of extracted ion chromatograms for the MW295 NOSs between this work and the work by Surratt et al (2008)

Figure S22. Correlation plots of MW297 peaks in ambient samples

Figure S23. Comparison of extracted ion chromatograms for the MW297 NOSs between this work and the work by Surratt et al (2008)

Figure S24. Correlation plots of MW363 peaks in ambient samples 
S1. Formation scheme of NOSs in the atmosphere and summary information of synthesized NOSs in this work
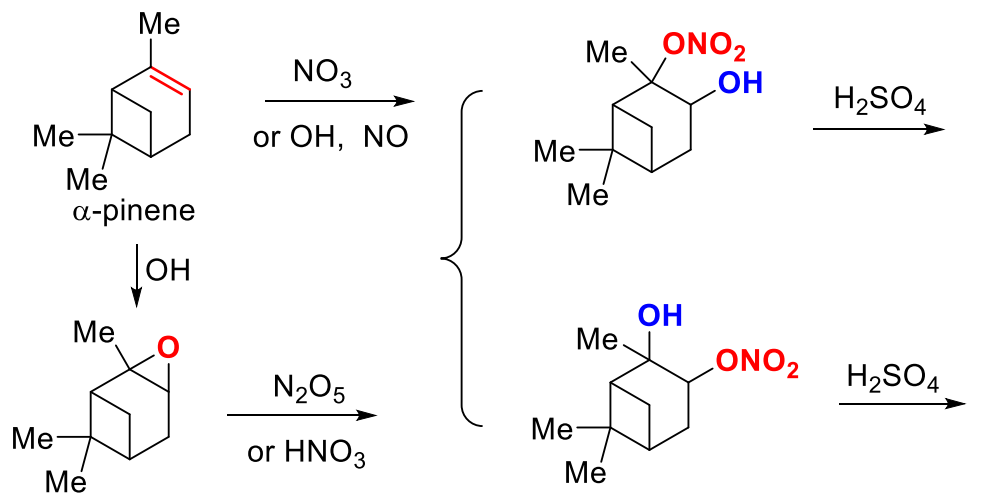<smiles>CC1(C)C2CC(OS(=O)(=O)O)C(C)(O[N+](=O)[O-])C1C2</smiles>

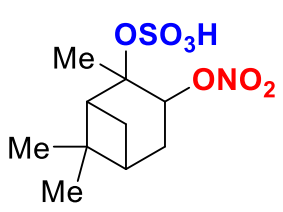

Scheme S1. General understanding of formation of NOS in atmosphere

Table S1. Summary information of nitrooxy organosulfates synthesized in this study

\begin{tabular}{|c|c|c|c|c|c|}
\hline Common Name & IUPAC name & formula & {$[\mathrm{M}-\mathrm{H}]^{-}$} & Structure & $\begin{array}{c}\text { Exact mass by } \\
\text { MALDI-MS }\end{array}$ \\
\hline $2^{\circ}$ ApNOS (4a') & $\begin{array}{c}\text { 1,7,7-trimethyl-6- } \\
\text { (nitrooxy)bicyclo[2.2.1 } \\
\text { ]heptan-2-yl sulfate }\end{array}$ & $\mathrm{C}_{10} \mathrm{H}_{16} \mathrm{NO}_{7} \mathrm{~S}^{-}$ & 294.07 & $\mathrm{SO}_{3}$ & 294.0657 \\
\hline $1^{\circ} \_$BpNOS (4d) & $\begin{array}{c}2-(4- \\
\text { ((nitrooxy)methyl)cycl } \\
\text { ohex-3-en-1- } \\
\text { yl)propan-2-yl sulfate }\end{array}$ & $\mathrm{C}_{10} \mathrm{H}_{16} \mathrm{NO}_{7} \mathrm{~S}^{-}$ & 294.07 & $\mathrm{ONO}_{2}$ & 294.0642 \\
\hline $3^{\circ} \_$ApNOS (4a) & $\begin{array}{c}\text { 2-methyl-5-(2- } \\
\text { (nitrooxy)propan-2- } \\
\text { yl)cyclohex-2-en-1-yl } \\
\text { sulfate }\end{array}$ & $\mathrm{C}_{10} \mathrm{H}_{16} \mathrm{NO}_{7} \mathrm{~S}^{-}$ & 294.07 & & / \\
\hline $2^{\circ}{ }_{2} \operatorname{LmNOS}\left(\mathbf{4} \mathbf{b}^{\prime}\right)$ & $\begin{array}{l}\text { 1-methyl-2-(nitrooxy)- } \\
\text { 4-(prop-1-en-2- } \\
\text { yl)cyclohexyl sulfate }\end{array}$ & $\mathrm{C}_{10} \mathrm{H}_{16} \mathrm{NO}_{7} \mathrm{~S}^{-}$ & 294.07 & $\mathrm{Me}$ & 294.0630 \\
\hline $3^{\circ}{ }_{-} \operatorname{LmNOS}(\mathbf{4 b})$ & $\begin{array}{l}\text { 2-methyl-2-(nitrooxy)- } \\
\text { 5-(prop-1-en-2- } \\
\text { yl)cyclohexyl sulfate }\end{array}$ & $\mathrm{C}_{10} \mathrm{H}_{16} \mathrm{NO}_{7} \mathrm{~S}^{-}$ & 294.07 & Me & $294.0647^{*}$ \\
\hline $2^{\circ}{ }_{-} \operatorname{LkNOS}\left(\mathbf{5} \mathbf{b}^{\prime}\right)$ & $\begin{array}{l}\text { 4-acetyl-1-methyl-2- } \\
\text { (nitrooxy)cyclohexyl } \\
\text { sulfate }\end{array}$ & $\mathrm{C}_{9} \mathrm{H}_{14} \mathrm{NO}_{8} \mathrm{~S}^{-}$ & 296.04 & $\mathrm{Me}$ & 296.0448 \\
\hline $3^{\circ}{ }_{2} \operatorname{LkNOS}(\mathbf{5 b})$ & $\begin{array}{l}\text { 5-acetyl-2-methyl-2- } \\
\text { (nitrooxy)cyclohexyl } \\
\text { sulfate }\end{array}$ & $\mathrm{C}_{9} \mathrm{H}_{14} \mathrm{NO}_{8} \mathrm{~S}^{-}$ & 296.04 & $\mathrm{Me}$ & $296.0450^{*}$ \\
\hline $2^{\circ}{ }_{\text {B }} \mathrm{BcpNOS}(\mathbf{4 c})$ & $\begin{array}{l}\text { 1,1,7-trimethyl-3- } \\
\text { (nitrooxy)decahydro- } \\
\text { 3a,7- } \\
\text { methanocyclopenta[8] } \\
\text { annulen-6-yl sulfate }\end{array}$ & $\mathrm{C}_{15} \mathrm{H}_{24} \mathrm{NO}_{7} \mathrm{~S}^{-}$ & 362.13 & & 362.1283 \\
\hline
\end{tabular}

*. Exact mass for isomers. 


\section{S2. Experimental Section}

\section{S2.1 General}

Chemicals and synthesis reactions. Chemicals purchased from commercial sources were used without further purification. Unless otherwise noted, all reactions were performed in round bottom flask under an air atmosphere. Reactions were magnetically stirred and monitored by thin layer chromatography (TLC, $0.25 \mathrm{~mm}$ ) on Merck pre-coated silica gel plates. Flash chromatography was performed with silica gel 60 (particle size $0.040-0.062 \mathrm{~mm}$ ) supplied by Grace.

NMR analysis. NMR spectra were recorded on a Bruker AV-400 spectrometer; chemical shifts are reported in parts per million (ppm) as values relative to the internal chloroform $\left(7.27 \mathrm{ppm}\right.$ for ${ }^{1} \mathrm{H}$ and $77.23 \mathrm{ppm}$ for ${ }^{13} \mathrm{C}$ ) or the internal methanol $\left(3.31 \mathrm{ppm}\right.$ for ${ }^{1} \mathrm{H}$ and $49.03 \mathrm{ppm}$ for $\left.{ }^{13} \mathrm{C}\right)$. Abbreviations for signal coupling are as follows: s, singlet; $d$, doublet; $t$, triplet; q, quartet; $\mathrm{m}$, multiplet.

MALDI-MS analysis. High resolution mass spectra were measured at the Hong Kong University of Science and Technology Mass Spectrometry Service Center on a MALDI Micro MX mass spectrometer (API QSTAR XL System, Waters, Milford, MA, USA).

UHPLC/MS analysis conditions. Thermo Ultimate 3000 HPLC coupled with an orbitrap mass spectrometer (Q-Exactive Hybrid Quadrupole-Orbitrap mass spectrometer, Thermo scientific, USA) was used in this work. Chromatographic separation was performed on an Acquity UPLC HSS T3 column $(2.1 \mathrm{~mm} \times 100 \mathrm{~mm}, 1.8 \mu \mathrm{m}$ particle size; Waters, Milford, MA, USA) with Van Guard column (HSS T3, $1.8 \mu \mathrm{m}$ ) at a flow rate of $0.3 \mathrm{~mL} / \mathrm{min}$. The mobile phase consisted of water (eluent A) and methanol (eluent B), each containing $0.1 \%$ acetic acid. The gradient elution program was as follows: eluent B initially set at $1 \%$ for $2.7 \mathrm{~min}$, increased to $54 \%$ in $15.2 \mathrm{~min}$ and held for $1 \mathrm{~min}$, then increased to $90 \%$ in $7.5 \mathrm{~min}$ and held for $0.2 \mathrm{~min}$; and finally decreased to $1 \%$ in $1.8 \mathrm{~min}$ and held for $9.3 \mathrm{~min}$ The Orbitrap mass spectrometer was operated in negative ESI mode under the following conditions: sheath gas flow at $35.0 \mathrm{~L} \mathrm{~min}{ }^{-1}$, spray voltage at $3.2 \mathrm{kV}$, S-lens RF level at 50.0, and temperature at $320{ }^{\circ} \mathrm{C}$. Calibration curves for LC/MS analysis were based on the $[\mathrm{M}-\mathrm{H}]^{-}$molecular ions in the extracted ion chromatogram (EIC) (abbreviated as EIC quantification thereafter) in the concentration range of 10 - 1000 $\mathrm{ng} / \mathrm{mL}$ and using $\mathrm{D}_{17}$-octyl sulfate as an internal standard.

MS/MS analysis. For the MS/MS data acquisition, a full MS scan (without collisional activation applied) was followed by a set of Data Dependent ${ }^{\mathrm{TM}}$ scans with stepped collision energy (CE) of 20, 40, and $60 \mathrm{ev}$ in the higher-energy collisional dissociation (HCD) cell. Fragments produced at the three CE events were combined and detected simultaneously to produce a combined MS/MS spectrum. The $m / z$ range was $90-500$ Da for full MS scan and 50-500 Da for the MS/MS data acquisition. Mass resolution was 140,000 in the full MS scan mode and 35,000 in the MS/MS scan mode.

\section{S2.2 General procedure for the preparation of terpene oxide ${ }^{2}$}

The terpene oxides were prepared according to the literature procedures. Solid $\mathrm{KHCO}_{3}(3.03 \mathrm{~g}, 30$ $\mathrm{mmol})$ was suspended in a solution of the terpene $(2.04 \mathrm{~g}, 15.0 \mathrm{~mol})$ and DCC $(6.19 \mathrm{~g}, 30 \mathrm{mmol})$ in 50 $\mathrm{mL}$ of methanol. Hydrogen peroxide ( $13 \mathrm{~mL}$ of a $50 \%$ solution, $180 \mathrm{mmol}$ ) was added, and the reaction mixture was stirred at room temperature for $24 \mathrm{~h}$. During the workup, the solids were removed by filtration, and the filtrate was quenched by $\mathrm{Na}_{2} \mathrm{~S}_{2} \mathrm{O}_{3}$ and dried by $\mathrm{Na}_{2} \mathrm{SO}_{4}$. After being concentrated under reduced pressure, the resulting residue was purified by flash column chromatography on silica gel (hexane/EtOAc $=18: 1$ ) to afford the desired epoxide. The reaction process was monitored by TLC using vanillin as color-developing agent. 


\section{Preparation of $\alpha$-pinene oxide (2a)}

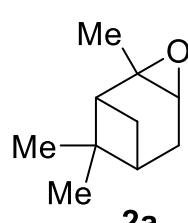

$2 a$

Use (+)- $\alpha$-pinene 1a $(2.04 \mathrm{~g}, 15 \mathrm{mmol})$ as the starting material and follow the representative procedure. Colorless liquid, $1.87 \mathrm{~g}$, yield: $82 \% .{ }^{1} \mathrm{H}$ NMR $(400 \mathrm{MHz}$, $\left.\mathrm{CD}_{3} \mathrm{OD}\right) \delta 4.41(\mathrm{~d}, J=1.7 \mathrm{~Hz}, 1 \mathrm{H}), 3.38-3.32(\mathrm{~m}, 1 \mathrm{H}), 3.29-3.24(\mathrm{~m}, 3 \mathrm{H}), 3.08-$ $3.00(\mathrm{~m}, 1 \mathrm{H}), 2.94(\mathrm{~d}, J=9.5 \mathrm{~Hz}, 1 \mathrm{H}), 2.64(\mathrm{~d}, J=3.0 \mathrm{~Hz}, 6 \mathrm{H}), 2.29(\mathrm{~s}, 3 \mathrm{H}) \cdot{ }^{13} \mathrm{C}$ NMR (101 MHz, CD $\left.{ }_{3} \mathrm{OD}\right) \delta 61.5,57.9,46.2,41.6,41.0,28.6,27.2,26.7,22.8,20.7$.

\section{Preparation of limonene oxide (2b)}

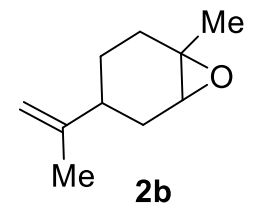

Use (+)-limonene $\mathbf{1 b}(2.04 \mathrm{~g}, 15 \mathrm{mmol})$ as the starting material and follow the representative procedure. Colorless liquid, $1.99 \mathrm{~g}$, yield: $87 \% .{ }^{1} \mathrm{H} \mathrm{NMR}(400 \mathrm{MHz}$, $\left.\mathrm{CDCl}_{3}\right) \delta 4.67-4.63(\mathrm{~m}, 0.6 \mathrm{H}), 4.58(\mathrm{~s}, 2 \mathrm{H}), 2.97$ (s, 0.3H), 2.91 (d, $J=5.2 \mathrm{~Hz}$, $1 \mathrm{H}), 2.10-2.00(\mathrm{~m}, 1 \mathrm{H}), 1.95$ (ddd, $J=13.9,7.3,2.4 \mathrm{~Hz}, 2 \mathrm{H}), 1.79$ (ddd, $J=10.8$, 9.5, 5.0 Hz, 2H), $1.64(\mathrm{dd}, J=7.6,2.3 \mathrm{~Hz}, 2 \mathrm{H}), 1.61(\mathrm{~s}, 1 \mathrm{H}), 1.60-1.55(\mathrm{~m}, 3 \mathrm{H})$, $1.51-1.40(\mathrm{~m}, 0.6 \mathrm{H}), 1.30(\mathrm{dd}, J=6.2,3.6 \mathrm{~Hz}, 1 \mathrm{H}), 1.24(\mathrm{~d}, J=1.5 \mathrm{~Hz}, 3 \mathrm{H}), 1.22(\mathrm{~d}, J=1.5 \mathrm{~Hz}, 1 \mathrm{H})$, $1.19-1.06(\mathrm{~m}, 0.6 \mathrm{H}) .{ }^{13} \mathrm{C} \mathrm{NMR}\left(101 \mathrm{MHz}, \mathrm{CDCl}_{3}\right) \delta 148.9,148.7,108.9,108.9,60.3,59.0,57.3,57.1$, $40.6,36.0,30.8,30.5,29.7,28.4,25.7,24.2,24.1,22.9,20.9,20.1$.

\section{Preparation of $\beta$-caryophyllene oxide (2c)}

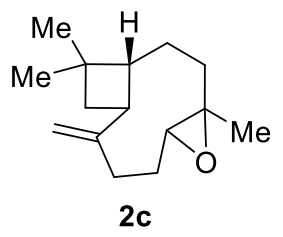

Use $\beta$-caryophyllene $1 \mathrm{c}(3.07 \mathrm{~g}, 15 \mathrm{mmol})$ as the starting material and follow the representative procedure. Light yellow solid, $2.68 \mathrm{~g}$, yield: $81 \%$. ${ }^{1} \mathrm{H}$ NMR (400 $\left.\mathrm{MHz}, \mathrm{CDCl}_{3}\right) \delta 4.96(\mathrm{~d}, J=7.6 \mathrm{~Hz}, 1 \mathrm{H}), 4.84(\mathrm{~d}, J=7.9 \mathrm{~Hz}, 1 \mathrm{H}), 2.94-2.78(\mathrm{~m}$, $1 \mathrm{H}), 2.61(\mathrm{dd}, J=18.1,8.8 \mathrm{~Hz}, 1 \mathrm{H}), 2.39-2.27(\mathrm{~m}, 1 \mathrm{H}), 2.26-2.15(\mathrm{~m}, 1 \mathrm{H})$, $2.14-2.02(\mathrm{~m}, 2 \mathrm{H}), 1.68$ (dddd, $J=27.5,19.0,17.8,9.3 \mathrm{~Hz}, 5 \mathrm{H}), 1.51-1.35$ (m, $1 \mathrm{H}), 1.35-1.23(\mathrm{~m}, 1 \mathrm{H}), 1.19(\mathrm{t}, J=4.7 \mathrm{~Hz}, 3 \mathrm{H}), 0.99(\mathrm{dd}, J=8.6,1.5 \mathrm{~Hz}, 6 \mathrm{H}) .{ }^{13} \mathrm{C}$ NMR $(101 \mathrm{MHz}$, $\left.\mathrm{CDCl}_{3}\right) \delta 151.7,112.7,63.7,59.8,50.6,48.7,39.7,39.1,33.9,30.1,29.8,27.1,21.6,16.9$.

\section{S2.3 General procedure for the preparation of Bromohydrins $\mathrm{s}^{3,4}$ :}

The Bromohydrins were prepared according to the literature procedures. Bromohydrins were prepared by reacting the corresponding $(+)$-limonene $(1.36 \mathrm{~g}, 10 \mathrm{mmol})$ with $\mathrm{N}$-Bromosuccinimide $(1.78$ $\mathrm{g}, 10 \mathrm{mmol})$ in aq acetone $\left(\mathrm{H}_{2} \mathrm{O} /\right.$ Acetone $\left.=1: 5\right)$ at $0{ }^{\circ} \mathrm{C}$. The reaction mixture was stirred for $30 \mathrm{~min}$ at $0{ }^{\circ} \mathrm{C}$. After completion of the reaction, the residue was washed with water. Then the organic phase was collected and the aqueous phase was extracted with $\mathrm{CH}_{2} \mathrm{Cl}_{2}(3 \times 15 \mathrm{~mL})$. The combined organic fractions was dried over anhydrous $\mathrm{MgSO}_{4}$ and concentrated under reduced pressure. The resulting residue was purified by flash column chromatography on silica gel (hexane/EtOAc $=12: 1$ ) to afford the desired Bromohydrins. The reaction process was monitored by TLC using Phosphomolybdic acid as color-developing agent.

Preparation of 2-bromo-1-methyl-4-(prop-1-en-2-yl)cyclohexanol (6b). Use (+)-limonene 1b (1.36<smiles>C=C(C)C1CCC(C)(O)C(Br)C1</smiles>

6b

$\mathrm{g}, 10 \mathrm{mmol})$ as the starting material and follow the representative procedure. Colorless liquid, $1.93 \mathrm{~g}$, yield: $83 \%{ }^{1} \mathrm{H}$ NMR $\left(400 \mathrm{MHz}, \mathrm{CDCl}_{3}\right) \delta 4.71(\mathrm{~d}, J=9.9$ $\mathrm{Hz}, 2 \mathrm{H}), 4.14$ (s, 1H), 2.47 (s, 1H), 2.40 (d, $J=4.7 \mathrm{~Hz}, 1 \mathrm{H}), 2.21$ (ddd, $J=14.2$, 11.0, 3.2 Hz, 1H), 1.92 (dtd, $J=11.8,7.8,3.8 \mathrm{~Hz}, 2 \mathrm{H}), 1.69$ (s, 3H), 1.57 (dd, $J=$ 4.8, 3.4 Hz, 3H), 1.36 (s, 3H). ${ }^{13} \mathrm{C}$ NMR (101 MHz, $\left.\mathrm{CDCl}_{3}\right) \delta 148.3,109.4,71.6$, $60.0,38.2,35.5,33.0,29.3,25.9,21.2$. The data were in agreement with the 
literature values ${ }^{3,4}$.

Preparation of 2-(4-(bromomethyl)cyclohex-3-en-1-yl)propan-2-ol (6d). Use (-)- $\beta$-pinene 1d (2.04

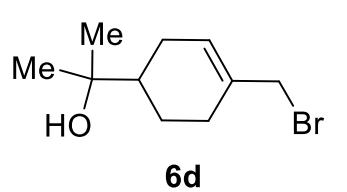

$\mathrm{g}, 15 \mathrm{mmol})$ as the starting material and follow the representative procedure. Colorless liquid, $1.93 \mathrm{~g}$, yield: $56 \% .{ }^{1} \mathrm{H}$ NMR $\left(400 \mathrm{MHz}, \mathrm{CDCl}_{3}\right) \delta 5.80(\mathrm{~s}, 1 \mathrm{H})$, $3.92-3.82(\mathrm{~m}, 2 \mathrm{H}), 2.17-2.08(\mathrm{~m}, 2 \mathrm{H}), 2.06(\mathrm{~s}, 1 \mathrm{H}), 1.90(\mathrm{dd}, J=12.5,2.4$ $\mathrm{Hz}, 1 \mathrm{H}), 1.83-1.68(\mathrm{~m}, 1 \mathrm{H}), 1.45(\mathrm{ddd}, J=11.9,4.7,2.4 \mathrm{~Hz}, 1 \mathrm{H}), 1.25-1.14$ $(\mathrm{m}, 1 \mathrm{H}), 1.10(\mathrm{~d}, J=4.3 \mathrm{~Hz}, 6 \mathrm{H}) .{ }^{13} \mathrm{C} \mathrm{NMR}\left(101 \mathrm{MHz}, \mathrm{CDCl}_{3}\right) \delta 134.2,127.5,72.1,44.2,38.9,27.0$, 27.0, 26.8, 26.1, 23.2. HRMS (TOF, $\left.\mathrm{LD}^{-}\right) \mathrm{m} / z$ calcd for $\mathrm{C}_{10} \mathrm{H}_{21} \mathrm{BrNO},\left[\mathrm{M}+\mathrm{NH}_{4}\right]^{+} 250.0807$, found 250.0798 .

\section{S2.4 General procedure for the preparation of hydroxynitrate ${ }^{5-11}$ :}

\section{Procedure A:}

Silver nitrate $(1.53 \mathrm{~g}, 9.0 \mathrm{mmol})$ was added to a solution of bromide $(3.0 \mathrm{mmol})$ in $\mathrm{CH}_{3} \mathrm{CN}(8 \mathrm{~mL})$ and stirred for $8 \mathrm{~h}$ under reflux. The mixture was poured onto brine $(50 \mathrm{~mL})$ to quench to remove excess $\mathrm{AgNO}_{3}$. The organic phase was collected and the aqueous phase was extracted with diethyl ether $(3 \times$ $30 \mathrm{~mL}$ ). The combined organic fractions were dried over anhydrous $\mathrm{MgSO}_{4}$ and concentrated under reduced pressure. The resulting residue was purified by flash column chromatography on silica gel (hexane/EtOAc $=12: 1$ ) to afford the desired hydroxyl nitrate. The reaction process was monitored by TLC using Phosphomolybdic acid as color-developing agent.

\section{Procedure B:}

The hydroxy nitrates were prepared according to the literature procedures. To a flame dried $25 \mathrm{~mL}$ round bottom flask equipped with a stir bar was added $(+)$-limonene oxide $(0.99 \mathrm{~g}, 6.52 \mathrm{mmols})$ and 8 $\mathrm{mL}$ dry diethyl ether (starting materials for the other standards were $\alpha$-pinene oxide $(0.99 \mathrm{~g})$ and Caryophyllene oxide $(1.44 \mathrm{~g})$ ). To another flame dried $10 \mathrm{~mL}$ round bottom flask equipped with a stir bar was added $3 \mathrm{~mL}$ dry diethyl ether and fuming nitric acid $(0.3 \mathrm{~mL}, 7.39 \mathrm{mmol})$. These flasks were placed under nitrogen, and cooled to $-94{ }^{\circ} \mathrm{C}$ via a hexane/liquid nitrogen bath. After cannulating the acid into the epoxide, the solution was kept at $-94^{\circ} \mathrm{C}$ for an additional hour. The reaction flask was then allowed to warm to room temperature overnight. The solution was quenched by the addition of saturated aqueous $\mathrm{NaHCO}_{3}(50 \mathrm{~mL})$ to remove excess $\mathrm{HNO}_{3}$. The organic phase was collected and the aqueous phase was extracted with diethyl ether $(3 \times 30 \mathrm{~mL})$. The combined organic fractions were dried over anhydrous $\mathrm{MgSO}_{4}$ and concentrated under reduced pressure. The resulting residue was purified by flash column chromatography on silica gel (hexane/EtOAc $=15: 1$ ) to afford the desired hydroxyl nitrate. The reaction process was monitored by TLC using phosphomolybdic acid as color-developing agent.

Fuming nitric acid is a strong and hazardous acid that is corrosive to the respiratory tract. The hazard statements are $\mathrm{H} 272, \mathrm{H} 290, \mathrm{H} 314, \mathrm{H} 331$, and $\mathrm{H} 335$. The precautionary statements are $\mathrm{P} 210$, $\mathrm{P} 220, \mathrm{P} 280, \mathrm{P} 303+\mathrm{P} 361+\mathrm{P} 353, \mathrm{P} 304+\mathrm{P} 340+\mathrm{P} 310, \mathrm{P} 305+\mathrm{P} 351+\mathrm{P} 338+\mathrm{P} 310, \mathrm{P} 370+\mathrm{P} 378$, and $\mathrm{P} 403+\mathrm{P} 233$.

\section{Preparation of 2-hydroxy-2-methyl-5-(prop-1-en-2-yl)cyclohexyl nitrate (3b').}<smiles>C=C(C)C1CCC(O)([N+]([O-])([O-])[O-])C(O[N+](=O)[O-])C1</smiles>

Use 2-bromo-1-methyl-4-(prop-1-en-2-yl)cyclohexanol $6 \mathbf{b}(0.70 \mathrm{~g}, 3.0 \mathrm{mmol})$ as the starting material and follow the same procedure described for Procedure A. Yellow liquid, $355.2 \mathrm{mg}$, yield: 55\%. ${ }^{1} \mathrm{H} \mathrm{NMR}\left(400 \mathrm{MHz}, \mathrm{CD}_{3} \mathrm{OD}\right) \delta 4.98(\mathrm{~s}, 1 \mathrm{H}), 4.73$ (s, 2H), $2.14(\mathrm{~s}, 1 \mathrm{H}), 2.09-1.99(\mathrm{~m}, 1 \mathrm{H}), 1.87(\mathrm{~d}, J=1.8 \mathrm{~Hz}, 1 \mathrm{H}), 1.71(\mathrm{~s}, 3 \mathrm{H}), 1.68$ - 
$1.52(\mathrm{~m}, 4 \mathrm{H}), 1.23(\mathrm{~s}, 3 \mathrm{H}) .{ }^{13} \mathrm{C} \mathrm{NMR}\left(101 \mathrm{MHz}, \mathrm{CD}_{3} \mathrm{OD}\right) \delta 149.8,109.9,85.7,69.7,39.5,35.6,30.9$, 26.9, 26.8, 21.0. HRMS (TOF, $\mathrm{Cl}^{-}$) $\mathrm{m} / z$ calcd for $\mathrm{C}_{10} \mathrm{H}_{16} \mathrm{NO}_{4}^{-},[\mathrm{M}-\mathrm{H}]^{+} 214.1085$, found 214.1073. The data were in agreement with the literature values. ${ }^{12}$

\section{Preparation of (4-(2-hydroxypropan-2-yl)cyclohex-1-en-1-yl)methyl nitrate (3d).}

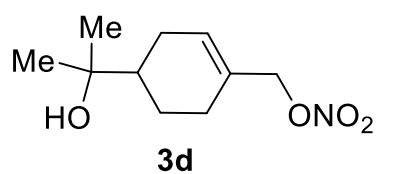

Use 2-(4-(bromomethyl)cyclohex-3-en-1-yl)propan-2-ol 6d (648.3 mg, 2.76 $\mathrm{mmol})$ as the starting material and follow the same procedure described for Procedure A. Yellow liquid, $124.2 \mathrm{mg}$, yield: $21 \% .{ }^{1} \mathrm{H}$ NMR $(400 \mathrm{MHz}$, $\left.\mathrm{CD}_{3} \mathrm{OD}\right) \delta 5.96-5.87(\mathrm{~m}, 1 \mathrm{H}), 4.86(\mathrm{~d}, J=3.7 \mathrm{~Hz}, 2 \mathrm{H}), 2.25-2.07(\mathrm{~m}, 3 \mathrm{H})$, $2.02-1.83(\mathrm{~m}, 2 \mathrm{H}), 1.57-1.48(\mathrm{~m}, 1 \mathrm{H}), 1.32-1.27(\mathrm{~m}, 1 \mathrm{H}), 1.17(\mathrm{t}, J=4.1 \mathrm{~Hz}, 6 \mathrm{H}) .{ }^{13} \mathrm{C}$ NMR $(101$ $\left.\mathrm{MHz}, \mathrm{CD}_{3} \mathrm{OD}\right) \delta 131.4,131.2,78.5,72.9,45.8,28.0,27.9,27.1,26.3,24.7$. HRMS (TOF, $\mathrm{Cl}^{-}$) $\mathrm{m} / \mathrm{z}$ calcd for $\mathrm{C}_{10} \mathrm{H}_{16} \mathrm{NO}_{4}^{-},[\mathrm{M}-\mathrm{H}]^{+} 214.1085$, found 214.1083 .

Preparation of 2-hydroxy-2-methyl-5-(prop-1-en-2-yl)cyclohexyl nitrate (3b') and 2-hydroxy-1methyl-4-(prop-1-en-2-yl)cyclohexyl nitrate (3b).

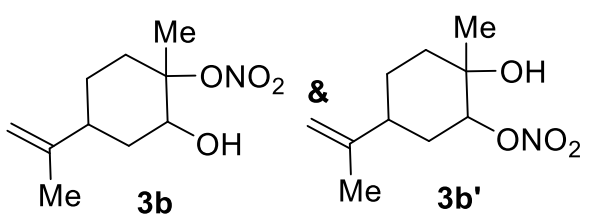

Use limonene oxide $\mathbf{2 b}(0.99 \mathrm{~g}, 6.5 \mathrm{mmol})$ as the starting material and follow the same procedure described for Procedure B. Yellow liquid, $1.04 \mathrm{~g}$, yield: $74 \% .3 \mathbf{b}: 3 \mathbf{b}^{\prime}=0.8: 1$. ${ }^{1} \mathrm{H} \mathrm{NMR}\left(400 \mathrm{MHz}, \mathrm{CDCl}_{3}\right) \boldsymbol{\delta} 5.00(\mathrm{t}, \mathrm{J}=2.7 \mathrm{~Hz}, 1 \mathrm{H}), 4.76(\mathrm{~s}$, $2 \mathrm{H}), 4.75-4.72(\mathrm{~m}, 1.6 \mathrm{H}), 4.11(\mathrm{~s}, 0.8 \mathrm{H}), 2.42-2.30(\mathrm{~m}$, $0.8 \mathrm{H}), 2.22-2.12(\mathrm{~m}, 1 \mathrm{H}), 2.02(\mathrm{ddd}, \mathrm{J}=14.7,12.1,2.6 \mathrm{~Hz}, 1 \mathrm{H}), 1.93(\mathrm{ddd}, \mathrm{J}=10.6,5.9,3.0 \mathrm{~Hz}, 1 \mathrm{H})$, $1.89-1.86(\mathrm{~m}, 0.8 \mathrm{H}), 1.84-1.77(\mathrm{~m}, 1.6 \mathrm{H}), 1.73(\mathrm{~s}, 5.4 \mathrm{H}), 1.69-1.61(\mathrm{~m}, 6.4 \mathrm{H}), 1.59-1.45(\mathrm{~m}$, 2.4H), $1.31(\mathrm{~s}, 3 \mathrm{H}) .{ }^{13} \mathrm{C}$ NMR $\left(101 \mathrm{MHz}, \mathrm{CDCl}_{3}\right) \delta 148.5,148.3,109.6,109.5,91.4,84.4,69.6,69.2$, $38.0,36.8,34.8,33.9,29.9,29.9,27.0,25.8,25.9,21.0,20.9,20.9$. The data were in agreement with the literature values ${ }^{12}$.

Preparation of (6-hydroxy-1,7,7-trimethylbicyclo[2.2.1]heptan-2-yl) nitrate (3a') \& 2-methyl-5-

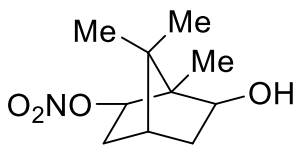

$3 a^{\prime}$<smiles>C=C(C)C1CC=C(C)C(O)C1</smiles>

Carveol (prop-1-en-2-yl)cyclohex-2-enol (carveol).

Use $\alpha$-pinene oxide $11 \mathrm{c}(0.99 \mathrm{~g}, 6.5 \mathrm{mmol})$ as the starting material and follow the same procedure described for Procedure B. Yellow liquid, $321.4 \mathrm{mg}$, yield: $23 \%$. 3a'/carveol $=1 / 1.5 .{ }^{1} \mathrm{H}$ NMR $(400$ $\mathrm{MHz}, \mathrm{CDCl} 3) \delta 5.57-5.46(\mathrm{~m}, 1.5 \mathrm{H}), 5.23(\mathrm{ddd}, \mathrm{J}=9.9,3.6,2.1$ $\mathrm{Hz}, 1 \mathrm{H}), 4.72-4.60$ (m, 3H), 4.04 (ddd, $\mathrm{J}=10.6,4.7,2.0 \mathrm{~Hz}$, $1 \mathrm{H}), 3.94(\mathrm{~s}, 1.5 \mathrm{H}), 2.67(\mathrm{~s}, 2.5 \mathrm{H}), 2.60-2.49(\mathrm{~m}, 1 \mathrm{H}), 2.44$ (dddd, $\mathrm{J}=13.9,10.6,4.9,3.5 \mathrm{~Hz}, 1 \mathrm{H}), 2.35-2.26(\mathrm{~m}, 1.5 \mathrm{H}), 2.07$ (ddd, $\mathrm{J}=16.0,6.3,2.6 \mathrm{~Hz}, 1.5 \mathrm{H}$ ), $1.90-1.76(\mathrm{~m}, 4 \mathrm{H}), 1.72(\mathrm{~d}, \mathrm{~J}=8.6 \mathrm{~Hz}, 4.5 \mathrm{H}), 1.68(\mathrm{~s}, 4.5 \mathrm{H}), 1.56-1.49(\mathrm{~m}, 1.5 \mathrm{H}), 1.36(\mathrm{dd}, \mathrm{J}=14.0$, $3.8 \mathrm{~Hz}, 1 \mathrm{H}), 1.19-1.13(\mathrm{~m}, 1 \mathrm{H}), 1.02(\mathrm{~s}, 3 \mathrm{H}), 0.89(\mathrm{~s}, 3 \mathrm{H}), 0.86(\mathrm{~s}, 3 \mathrm{H}) .13 \mathrm{C}$ NMR (101 MHz, CDCl3) $\delta$ 149.2, 134.4, 125.0, 108.8, 90.8, 76.9, 68.1, 52.5, 49.4, 42.7, 38.8, 36.7, 36.5, 35.1, 31.0, 20.8, 20.1, 19.6, 11.7. The data were in agreement with the literature values ${ }^{12}$.

Preparation of 2-(5-hydroxy-4-methylcyclohex-3-en-1-yl)propan-2-yl nitrate (3a). 


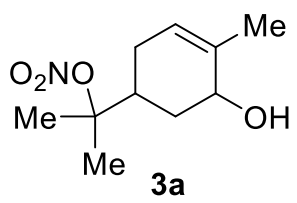

Use $\alpha$-pinene oxide $11 \mathrm{c}(0.99 \mathrm{~g}, 6.5 \mathrm{mmol})$ as the starting material and follow the same procedure described for Procedure B. Yellow liquid, $125.1 \mathrm{mg}$, yield: 8.9\%. ${ }^{1} \mathrm{H}$ NMR (400 MHz, $\left.\mathrm{CDCl}_{3}\right) \delta 5.54(\mathrm{dd}, J=3.7,1.6 \mathrm{~Hz}, 1 \mathrm{H}), 4.03(\mathrm{~s}, 1 \mathrm{H}), 3.02$ (s, 1H), 2.41- $2.27(\mathrm{~m}, 1 \mathrm{H}), 2.07$ (ddd, $J=13.5,10.2,5.1 \mathrm{~Hz}, 1 \mathrm{H}), 1.97-1.84(\mathrm{~m}$, $3 \mathrm{H}), 1.78(\mathrm{~s}, 3 \mathrm{H}), 1.55(\mathrm{~d}, J=6.2 \mathrm{~Hz}, 3 \mathrm{H}), 1.51(\mathrm{~s}, 3 \mathrm{H}) \cdot{ }^{13} \mathrm{C} \mathrm{NMR}(101 \mathrm{MHz}$, $\left.\mathrm{CDCl}_{3}\right) \delta 134.4,124.3,94.4,68.0,36.1,32.7,26.5,22.5,22.4,20.8$. HRMS (TOF, $\mathrm{Cl}^{--}$) $\mathrm{m} / \mathrm{z}$ calcd for $\mathrm{C}_{10} \mathrm{H}_{16} \mathrm{NO}_{4}^{-},[\mathrm{M}-\mathrm{H}]^{+} 214.1085$, found 214.1081. The data were in agreement with the literature values ${ }^{12}$.

\section{Preparation of 2-(2,2,3-trimethylcyclopent-3-en-1-yl)-acetaldehyde (Campholenaldehyde).}

Use $\alpha$-pinene oxide 11c ( $0.99 \mathrm{~g}, 6.5 \mathrm{mmol})$ as the starting material and follow<smiles>CC1=CCC(C=O)C1(C)C</smiles>

Campholenaldehyde the same procedure described for Procedure B. Yellow liquid, $236.3 \mathrm{mg}$, yield: 17\%. ${ }^{1} \mathrm{H}$ NMR (400 MHz, $\left.\mathrm{CDCl}_{3}\right) \delta 9.74(\mathrm{dd}, J=4.2,2.1 \mathrm{~Hz}, 1 \mathrm{H}), 5.17$ (s, $1 \mathrm{H}), 2.51-2.42(\mathrm{~m}, 1 \mathrm{H}), 2.38-2.29(\mathrm{~m}, 2 \mathrm{H}), 2.25-2.15(\mathrm{~m}, 1 \mathrm{H}), 1.86-1.78$

$(\mathrm{m}, 1 \mathrm{H}), 1.56(\mathrm{dd}, J=4.0,1.5 \mathrm{~Hz}, 3 \mathrm{H}), 0.94(\mathrm{~d}, J=3.6 \mathrm{~Hz}, 3 \mathrm{H}), 0.74(\mathrm{~d}, J=$

$3.5 \mathrm{~Hz}, 3 \mathrm{H}) .{ }^{13} \mathrm{C}$ NMR $\left(101 \mathrm{MHz}, \mathrm{CDCl}_{3}\right) \delta 202.7,147.8,121.5,46.8,45.0,44.1,35.5,25.5,19.9,12.5$. The data were in agreement with the literature values ${ }^{12}$

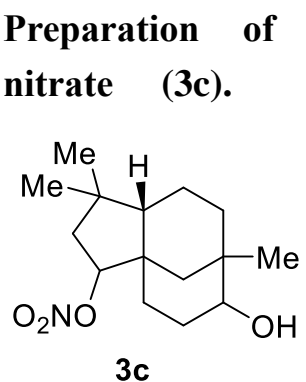

\section{6-hydroxy-1,1,7-trimethyldecahydro-3a,7-methanocyclopenta[8]annulen-3-yl}

Use Caryophyllene oxide $\mathbf{3 b}(1.44 \mathrm{~g}, 6.5 \mathrm{mmol})$ as the starting material and follow the same procedure described for Procedure B. Yellow liquid, $85.8 \mathrm{mg}$, yield: $5 \% .{ }^{1} \mathrm{H}$ NMR $\left(400 \mathrm{MHz}, \mathrm{CDCl}_{3}\right) \delta 4.99(\mathrm{dd}, J=8.3,5.9 \mathrm{~Hz}, 1 \mathrm{H}), 3.31(\mathrm{~s}$, $1 \mathrm{H}), 2.02(\mathrm{~d}, J=5.1 \mathrm{~Hz}, 1 \mathrm{H}), 1.93(\mathrm{ddd}, J=18.7,10.3,4.9 \mathrm{~Hz}, 2 \mathrm{H}), 1.69-1.59$ (m, 4H), 1.50 (ddt, $J=12.8,6.2,4.5 \mathrm{~Hz}, 2 \mathrm{H}), 1.43-1.32(\mathrm{~m}, 2 \mathrm{H}), 1.23$ (td, $J=$ 7.0, $2.7 \mathrm{~Hz}, 2 \mathrm{H}), 1.06(\mathrm{~s}, 3 \mathrm{H}), 0.97-0.91(\mathrm{~m}, 6 \mathrm{H}) .{ }^{13} \mathrm{C} \mathrm{NMR}\left(101 \mathrm{MHz}, \mathrm{CDCl}_{3}\right)$ $\delta$ 91.5, 74.2, 50.2, 45.0, 42.8, 38.0, 35.3, 34.4, 32.6, 31.1, 28.1, 26.7, 26.2, 25.1, 20.6. HRMS (TOF, $\left.\mathrm{LD}^{+}\right) \mathrm{m} / z$ calcd for $\mathrm{C}_{15} \mathrm{H}_{25} \mathrm{NO}_{4} \mathrm{NH}_{4}+,\left[\mathrm{M}+\mathrm{NH}_{4}\right]^{+}$301.2122, found 301.2130.

\section{S2.5 Procedure for the preparation of nitrooxy organosulfate sodium salt ${ }^{13-16}$ :}

\section{Procedure A:}

Representative Procedure for Synthesis of Nitrooxyorganosulfates: To a solution of nitrate (2.3 mmol) in DMF ( $3 \mathrm{~mL})$ was added $\mathrm{SO}_{3}-\mathrm{Py}^{14,15}(1.1 \mathrm{~g}, 6.9 \mathrm{mmol})$. After stirring for $12 \mathrm{~h}$ at $40^{\circ} \mathrm{C}$, saturated aqueous $\mathrm{NaHCO}_{3}$ was added to quench the reaction and the resulting mixture was stirred for $30 \mathrm{~min}$. After filtration and evaporation of solvent, the crude mixture was purified by flash column chromatography on silica gel $\left(\mathrm{CH}_{2} \mathrm{Cl}_{2} / \mathrm{MeOH}=12: 1\right)$ to afford the corresponding sulfate sodium salt. The reaction process was monitored by TLC using phosphomolybdic acid as color-developing agent.

\section{Procedure B:}

To a solution of sulfate $(1.0 \mathrm{mmol})$ in the mixed solvent of $12 \mathrm{~mL} \mathrm{CH} \mathrm{Cl}_{2}$ and $\mathrm{MeOH}\left(\mathrm{CH}_{2} \mathrm{Cl}_{2}\right.$ : $\mathrm{MeOH}=5: 1)$ was added pyridine $(0.4 \mathrm{~mL}, 4.0 \mathrm{mmol}) .{ }^{16,17}$ The solution at $-78{ }^{\circ} \mathrm{C}$ was bubbled with a stream of ozone from ozone generator for $10 \mathrm{~min}$, and then oxygen gas went through the solution to quench the reaction. The reaction mixture was stirred for $2 \mathrm{~h}$ at room temperature. After evaporation of solvent, the crude mixture was purified by flash column chromatography on silica gel $\left(\mathrm{CH}_{2} \mathrm{Cl}_{2} / \mathrm{MeOH}\right.$ : 
$=12: 1)$ to afford corresponding product. In this process we used phosphomolybdic acid as a color developing agent and TLC to monitor the product.

Preparation of sodium 1-methyl-2-(nitrooxy)-4-(prop-1-en-2-yl)cyclohexyl sulfate $\left(4 b^{\prime}=\right.$<smiles>[Y6]C1(O[Na])CCC(C(=C)C)CC1[N+](=O)[O-]</smiles>
$\mathbf{2}^{\mathbf{0}}$ _LmNOS). Use 2-hydroxy-2-methyl-5-(prop-1-en-2-yl)cyclohexyl nitrate 3b' $(0.5 \mathrm{~g}, 2.3 \mathrm{mmol})$ as starting material and follow the representative procedure A. White solid, $408 \mathrm{mg}$, yield: $56 \%{ }^{1} \mathrm{H}$ NMR $\left(400 \mathrm{MHz}, \mathrm{CD}_{3} \mathrm{OD}\right) \delta 5.71(\mathrm{~s}, 1 \mathrm{H})$, $4.73(\mathrm{~d}, J=4.1 \mathrm{~Hz}, 2 \mathrm{H}), 2.19-2.12(\mathrm{~m}, 2 \mathrm{H}), 2.08(\mathrm{~d}, J=14.3 \mathrm{~Hz}, 1 \mathrm{H}), 1.88$ (d, 4b' = 2' ${ }^{\circ}$ LmNOS $\left.J=10.1 \mathrm{~Hz}, 1 \mathrm{H}\right), 1.73(\mathrm{~s}, 1 \mathrm{H}), 1.72(\mathrm{~s}, 3 \mathrm{H}), 1.60(\mathrm{~s}, 3 \mathrm{H}), 1.55(\mathrm{dd}, J=12.8,2.7$ $\mathrm{Hz}, 2 \mathrm{H}) .{ }^{13} \mathrm{C} \mathrm{NMR}\left(101 \mathrm{MHz}, \mathrm{CD}_{3} \mathrm{OD}\right) \delta 149.9,109.9,82.9,81.3,39.2,35.4,31.0,26.7,22.7,20.9$. HRMS (TOF, $\mathrm{Cl}^{-}$) $m / z$ calcd for $\mathrm{C}_{10} \mathrm{H}_{16} \mathrm{NO}_{7} \mathrm{~S}^{-},[\mathrm{M}-\mathrm{Na}]^{-} 294.0653$, found 294.0630.

Preparation of sodium 4-acetyl-1-methyl-2-(nitrooxy)cyclohexyl sulfate $\left(5 b^{\prime}=2^{\circ}{ }_{-}\right.$LkNOS). Use

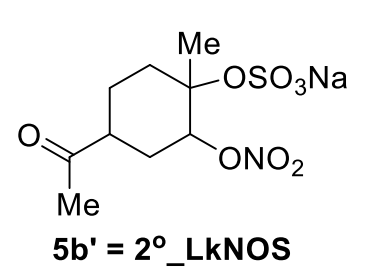
sodium 1-methyl-2-(nitrooxy)-4-(prop-1-en-2-yl)cyclohexyl sulfate $\mathbf{4} \mathbf{b}$ ' (190.2 $\mathrm{mg}, 0.6 \mathrm{mmol})$ as starting material and follow the representative procedure B. Yellow solid, $80.5 \mathrm{mg}$, yield: $42 \%{ }^{1} \mathrm{H}$ NMR $\left(400 \mathrm{MHz}, \mathrm{CD}_{3} \mathrm{OD}\right)$ $\delta 5.73(\mathrm{~s}, 1 \mathrm{H}), 2.68(\mathrm{td}, J=11.4,6.0 \mathrm{~Hz}, 1 \mathrm{H}), 2.29-2.22(\mathrm{~m}, 1 \mathrm{H}), 2.19$ (d, $J$ $=5.8 \mathrm{~Hz}, 3 \mathrm{H}), 2.12(\mathrm{~d}, J=13.9 \mathrm{~Hz}, 1 \mathrm{H}), 2.03(\mathrm{dd}, J=15.5,4.1 \mathrm{~Hz}, 1 \mathrm{H}), 1.82$ $(\mathrm{d}, J=9.9 \mathrm{~Hz}, 2 \mathrm{H}), 1.72-1.64(\mathrm{~m}, 1 \mathrm{H}), 1.62(\mathrm{~s}, 3 \mathrm{H}) .{ }^{13} \mathrm{C}$ NMR $(101 \mathrm{MHz}$, $\left.\mathrm{CD}_{3} \mathrm{OD}\right) \delta 213.1,82.1,81.2,45.5,34.6,28.1,27.6,24.0,22.2$. HRMS (TOF, $\mathrm{Cl}^{-}$) $\mathrm{m} / z$ calcd for $\mathrm{C}_{9} \mathrm{H}_{14} \mathrm{NO}_{8} \mathrm{~S}^{-},[\mathrm{M}-\mathrm{Na}]^{-} 296.0446$, found 296.0448.

Preparation of sodium 1-methyl-2-(nitrooxy)-4-(prop-1-en-2-yl)cyclohexyl sulfate $\left(4 b^{\prime}=\right.$<smiles>C=C(C)C1CCC([N+](=O)[O-])([N+](=O)[O-])C(O[N+](=O)[O-])C1</smiles>

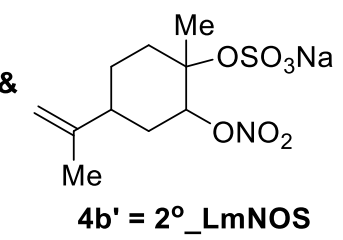
$2^{\circ}{ }_{-}$LmNOS) \& sodium 2-methyl-2-(nitrooxy)-5-(prop1-en-2-yl)cyclohexyl sulfate $\left(4 b=3^{0}\right.$ LmNOS $)$. Use mixture of 2-hydroxy-2-methyl-5-(prop-1-en-2yl)cyclohexyl nitrate $\mathbf{3 b}$ ' and 2-hydroxy-1-methyl-4(prop-1-en-2-yl)cyclohexyl nitrate 3b (215.3 g, $1.0 \mathrm{mmol}$ ) as starting material and follow the representative procedure A. Yellow liquid, $305.7 \mathrm{mg}$, yield: $96 \%$. 4b:4b' = 1:1. ${ }^{1} \mathrm{H}$ NMR $\left(400 \mathrm{MHz}, \mathrm{CD}_{3} \mathrm{OD}\right) \delta 5.72(\mathrm{~s}, 1 \mathrm{H}), 4.81(\mathrm{~s}, 1 \mathrm{H}), 4.75(\mathrm{~s}, 2 \mathrm{H}), 4.73(\mathrm{~d}, J=3.3$ $\mathrm{Hz}, 2 \mathrm{H}), 2.47-2.37(\mathrm{~m}, 1 \mathrm{H}), 2.31-2.05(\mathrm{~m}, 5 \mathrm{H}), 1.92-1.82(\mathrm{~m}, 2 \mathrm{H}), 1.79-1.75(\mathrm{~m}, 1 \mathrm{H}), 1.72(\mathrm{~d}, J$ $=8.6 \mathrm{~Hz}, 6 \mathrm{H}), 1.68(\mathrm{~s}, 3 \mathrm{H}), 1.64(\mathrm{~s}, 1 \mathrm{H}), 1.62(\mathrm{~d}, J=4.5 \mathrm{~Hz}, 4 \mathrm{H}), 1.58(\mathrm{~d}, J=2.0 \mathrm{~Hz}, 1 \mathrm{H}), 1.55(\mathrm{~d}, J$ $=3.0 \mathrm{~Hz}, 1 \mathrm{H}), 1.50-1.41(\mathrm{~m}, 1 \mathrm{H}), 1.17(\mathrm{~d}, J=6.2 \mathrm{~Hz}, 1 \mathrm{H}) \cdot{ }^{13} \mathrm{C}$ NMR $\left(101 \mathrm{MHz}, \mathrm{CD}_{3} \mathrm{OD}\right) \delta 149.9$, 149.8, 109.8, 109.6, 90.8, 82.8, 81.2, 76.1, 39.1, 38.1, 35.3, 32.3, 32.0, 30.9, 26.6, 22.6, 21.6, 21.0, 20.9. HRMS (TOF, $\mathrm{Cl}^{-}$) $\mathrm{m} / z$ calcd for $\mathrm{C}_{10} \mathrm{H}_{16} \mathrm{NO}_{7} \mathrm{~S}^{-}$, [M-Na] 294.0653 , found 294.0647.

Preparation of sodium 1,7,7-trimethyl-6-(nitrooxy)bicyclo[2.2.1]heptan-2-yl sulfate $\left(4 a^{\prime}=\right.$<smiles>CC1(C)C2CC(O[N+](=O)[O-])C(OS(=O)(=O)O[Na])CC1C2O</smiles>

$4 a^{\prime}=2^{\circ} \_$ApNOS $2^{\mathbf{0}}$ _ApNOS). Use mixture of 6-hydroxy-1,7,7-trimethylbicyclo[2.2.1]heptan-2-yl nitrate and 2-methyl-5-(prop-1-en-2-yl)cyclohex-2-enol (3a'\& Carveol, $236.3 \mathrm{mg}, 1.1 \mathrm{mmol}$ ) as starting material and follow the representative procedure A. Yellow solid, $237 \mathrm{mg}$, yield: $77 \% .{ }^{1} \mathrm{H}$ NMR $\left(400 \mathrm{MHz}, \mathrm{CD}_{3} \mathrm{OD}\right) \delta 5.31(\mathrm{~d}, J=6.7 \mathrm{~Hz}, 1 \mathrm{H}), 4.75(\mathrm{~d}, J=7.5 \mathrm{~Hz}, 1 \mathrm{H})$, 
$2.55(\mathrm{dd}, J=27.1,10.6 \mathrm{~Hz}, 2 \mathrm{H}), 1.87(\mathrm{~s}, 1 \mathrm{H}), 1.58(\mathrm{dd}, J=13.8,3.3 \mathrm{~Hz}, 1 \mathrm{H}), 1.42(\mathrm{dd}, J=13.3,2.8$ $\mathrm{Hz}, 1 \mathrm{H}), 1.12$ (s, 3H), 1.02 (s, 3H), 1.00 (s, 3H). ${ }^{13} \mathrm{C}$ NMR (101 MHz, $\left.\mathrm{CD}_{3} \mathrm{OD}\right) \delta 89.8,83.7,53.3,50.3$, 43.8, 37.1, 36.6, 20.3, 20.2, 12.4. HRMS (TOF, $\mathrm{Cl}^{-}$) $\mathrm{m} / z$ calcd for $\mathrm{C}_{10} \mathrm{H}_{16} \mathrm{NO}_{7} \mathrm{~S}^{-}$, [M-Na] 294.0653, found 294.0657 .

Preparation of sodium 1,1,7-trimethyl-3-(nitrooxy)decahydro-3a,7-methanocyclopenta[8] annulen-6-yl sulfate $\left(\mathbf{4 c}=\mathbf{2}^{\mathbf{0}}{ }_{-} \mathbf{B c p N O S}\right)$. Use 6-hydroxy-1,1,7-trimethyldecahydro-3a,7-

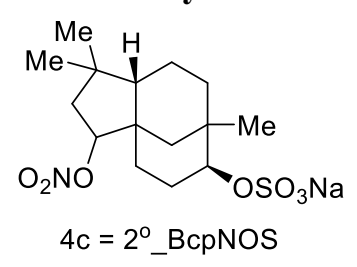
methanocyclopenta[8]annulen-3-yl nitrate $\mathbf{3 c}(85.8 \mathrm{mg}, 0.3 \mathrm{mmol})$ as starting material and follow the representative procedure A. Light yellow solid, 104.3 mg, yield: 90\%. ${ }^{1} \mathrm{H}$ NMR (400 MHz, $\left.\mathrm{CD}_{3} \mathrm{OD}\right) \delta 5.06(\mathrm{t}, J=6.5 \mathrm{~Hz}, 1 \mathrm{H}), 4.02$ (s, 1H), $2.21(\mathrm{~d}, J=14.5 \mathrm{~Hz}, 1 \mathrm{H}), 1.96(\mathrm{dd}, J=17.4,9.9 \mathrm{~Hz}, 2 \mathrm{H}), 1.79-1.64$ $(\mathrm{m}, 2 \mathrm{H}), 1.57(\mathrm{dd}, J=23.1,9.8 \mathrm{~Hz}, 3 \mathrm{H}), 1.52-1.40(\mathrm{~m}, 2 \mathrm{H}), 1.24(\mathrm{dd}, J=$ 28.1, $13.2 \mathrm{~Hz}, 3 \mathrm{H}), 1.10$ (s, 3H), 1.04 (s, 3H), 0.99 (s, 3H). ${ }^{13} \mathrm{C} \mathrm{NMR}\left(101 \mathrm{MHz}, \mathrm{CD}_{3} \mathrm{OD}\right) \delta 92.7,83.3$, 51.7, 46.1, 43.7, 38.9, 37.0, 35.2, 33.6, 31.4, 28.9, 28.2, 25.4, 24.4, 21.5. HRMS (TOF, $\mathrm{Cl}^{-}$) $\mathrm{m} / z$ calcd for $\mathrm{C}_{15} \mathrm{H}_{24} \mathrm{NO}_{7} \mathrm{~S}^{-}$, [M-Na] 362.1279 , found 362.1283.

\section{S2.6 Purity of compounds 4a' and 5b':}

In ${ }^{1} \mathrm{H}$ NMR spectra, the ratio of peak area represents the ratio of hydrogen atoms. Dichloroacetic acid has only one active hydrogen atom appearing as a single peak at chemical shift $6.2 \mathrm{ppm}$. For $2^{\circ} \_$ApNOS $(5.3 \mathrm{ppm})$ and $2^{\circ}{ }_{-}$LkNOSs $(5.7 \mathrm{ppm})$, we chose the hydrogen atom attached to the carbon atom that is bonded with the sulfate group for monitoring the quantity. The purity of $2^{\circ}{ }_{-}$ApNOS was determined by the peak areas in the ${ }^{1} \mathrm{H}$ NMR spectra of $2^{\circ}{ }_{2}$ ApNOS mixture $(40.3 \mathrm{mg}$ ) using dichloroacetic acid $(9.8 \mathrm{mg})$ in $\mathrm{CD}_{3} \mathrm{OD}$ as an internal standard. The peak area ratio of dichloroacetic acid to $2^{\circ}$ ApNOS is $0.62: 1$. Thus, the corresponding mass ratio is $1: 3.99$ and the mass of $2^{\circ}$ ApNOS is $39.1 \mathrm{mg}$ in the mixture. Hence, the purity is $97 \%$. The same precursor was used for compound $2^{\circ} \_$LkNOSs and the purity is $95.4 \%$. For other NOSs, we integrated all the peaks from the byproducts and determined their purities to be higher than $95 \%$. In summary, the purity of all the synthesized NOSs was higher than $95 \%$ based on NMR spectra data.

\section{References:}

(1) Surratt, J. D.; Gomez-Gonzalez, Y.; Chan, A. W. H.; Vermeylen, R.; Shahgholi, M.; Kleindienst, T. E.; Edney, E. O.; Offenberg, J. H.; Lewandowski, M.; Jaoui, M.; Maenhaut, W.; Claeys, M.; Flagan, R. C.; Seinfeld, J. H. Organosulfate Formation in Biogenic Secondary Organic Aerosol. J. Phys. Chem. A 2008, 112 (36), 8345-8378. https://doi.org/Doi 10.1021/Jp802310p.

(2) Majetich, G.; Hicks, R.; Sun, G. R.; McGill, P. Carbodiimide-Promoted Olefin Epoxidation with Aqueous Hydrogen Peroxide. J. Org. Chem. 1998, 63 (8), 2564-2573. https://doi.org/10.1021/jo972026n.

(3) Filler, R. Oxidations and Dehydrogenations with N-Bromosuccinimide and Related nHaloimides. Chem. Rev. 1963, 63 (1), 21-43. https://doi.org/10.1021/cr60221a002. 
(4) Kannan, N.; Rangaswamy, M. J.; Kemapaiah, B. B. Microwave Assisted Bi-Functional Activation of $\beta$-Bromo-Tert-Alcohols. J. Chem. Sci. 2015, 127 (8), 1405-1410. https://doi.org/10.1007/s12039-015-0906-y.

(5) Treves, K.; Rudich, Y. The Atmospheric Fate of C3-C6 Hydroxyalkyl Nitrates. J. Phys. Chem. A 2003, 107 (39), 7809-7817. https://doi.org/10.1021/jp0350641.

(6) Treves, K.; Shragina, L.; Rudich, Y. Henry's Law Constants of Some $\beta-, \gamma-$, and $\delta$-Hydroxy Alkyl Nitrates of Atmospheric Interest. Environ. Sci. Technol. 2000, 34 (7), 1197-1203. https://doi.org/10.1021/es990558a.

(7) Nortcliffe, A.; Ekstrom, A. G.; Black, J. R.; Ross, J. A.; Habib, F. K.; Botting, N. P.; O’Hagan, D. Synthesis and Biological Evaluation of Nitric Oxide-Donating Analogues of Sulindac for Prostate Cancer Treatment. Bioorganic Med. Chem. 2014, 22 (2), 756-761. https://doi.org/10.1016/j.bmc.2013.12.014.

(8) Yang, X.; Luo, F.; Li, J.; Chen, D.; Ye, E.; Lin, W.; Jin, J. Alkyl and Aromatic Nitrates in Atmospheric Particles Determined by Gas Chromatography Tandem Mass Spectrometry. J. Am. Soc. Mass Spectrom. 2019, 30 (12), 2762-2770. https://doi.org/10.1007/s13361-019-02347-8.

(9) Nichols, P. L.; Magnusson, A. B.; Ingham, J. D. Synthesis of Nitric Esters by the Addition of Nitric Acid to the Ethylene Oxide Ring. J. Am. Chem. Soc. 1953, 75 (17), 4255-4258. https://doi.org/10.1021/ja01113a031.

(10) Muthuramu, K.; Shepson, P. B.; O’Brien, J. M. Preparation, Analysis, and Atmospheric Production of Multifunctional Organic Nitrates. Environ. Sci. Technol. 1993, 27 (6), 1117-1124. https://doi.org/10.1021/es00043a010.

(11) Rollins, A. W.; Fry, J. L.; Hunter, J. F.; Kroll, J. H.; Worsnop, D. R.; Singaram, S. W.; Cohen, R. C. Elemental Analysis of Aerosol Organic Nitrates with Electron Ionization High-Resolution Mass Spectrometry. Atmos. Meas. Tech. 2010, 3 (1), 301-310. https://doi.org/10.5194/amt-3301-2010.

(12) Ali Mcknight, E.; P. Kretekos, N.; Owusu, D.; Lyn Lalonde, R. Technical Note: Preparation and Purification of Atmospherically Relevant Hydroxynitrate Esters of Monoterpenes. Atmos. Chem. Phys. 2020, 20 (7), 4241-4254. https://doi.org/10.5194/acp-20-4241-2020.

(13) Gilbert, E. E. The Reactions of Sulfur Trioxide, and of Its Adducts, with Organic Compounds. Chem. Rev. 1962, 62 (6), 549-589. https://doi.org/10.1021/cr60220a003.

(14) Wang, Y.; Ren, J.; Huang, X. H. H.; Tong, R.; Yu, J. Z. Synthesis of Four Monoterpene-Derived Organosulfates and Their Quantification in Atmospheric Aerosol Samples. Environ. Sci. Technol. 
2017, 51 (12), 6791-6801. https://doi.org/10.1021/acs.est.7b01179.

(15) Wang, Y.; Ma, Y.; Li, X.; Kuang, B. Y.; Huang, C.; Tong, R.; Yu, J. Z. Monoterpene and Sesquiterpene $\alpha$-Hydroxy Organosulfates: Synthesis, MS/MS Characteristics, and Ambient Presence. Environ. Sci. Technol. 2019, $53 \quad$ (21), 12278-12290. https://doi.org/10.1021/acs.est.9b04703.

(16) Bailey, P. S. The Reactions of Ozone with Organic Compounds. Chem. Rev. 1958, 58 (5), 9251010. https://doi.org/10.1021/cr50023a005. 


\section{S3. ${ }^{1} \mathrm{H}$ and ${ }^{13} \mathrm{C}$ NMR Spectra}

(a)

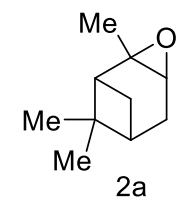

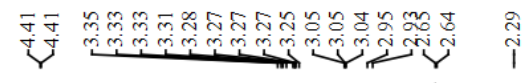

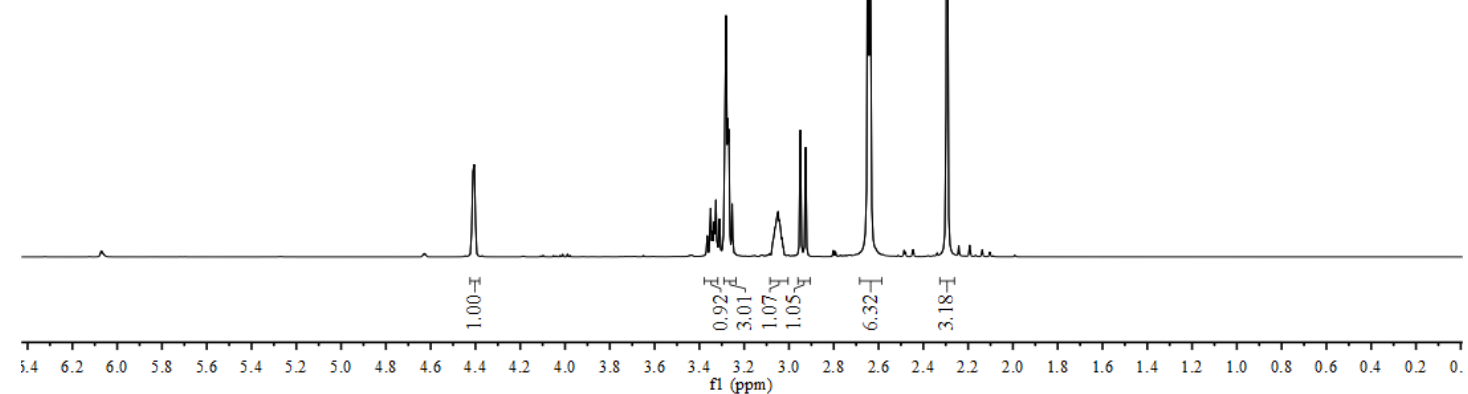

(b)

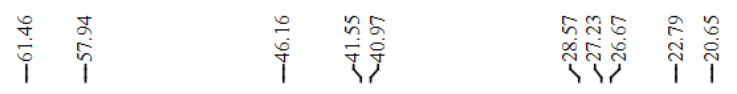
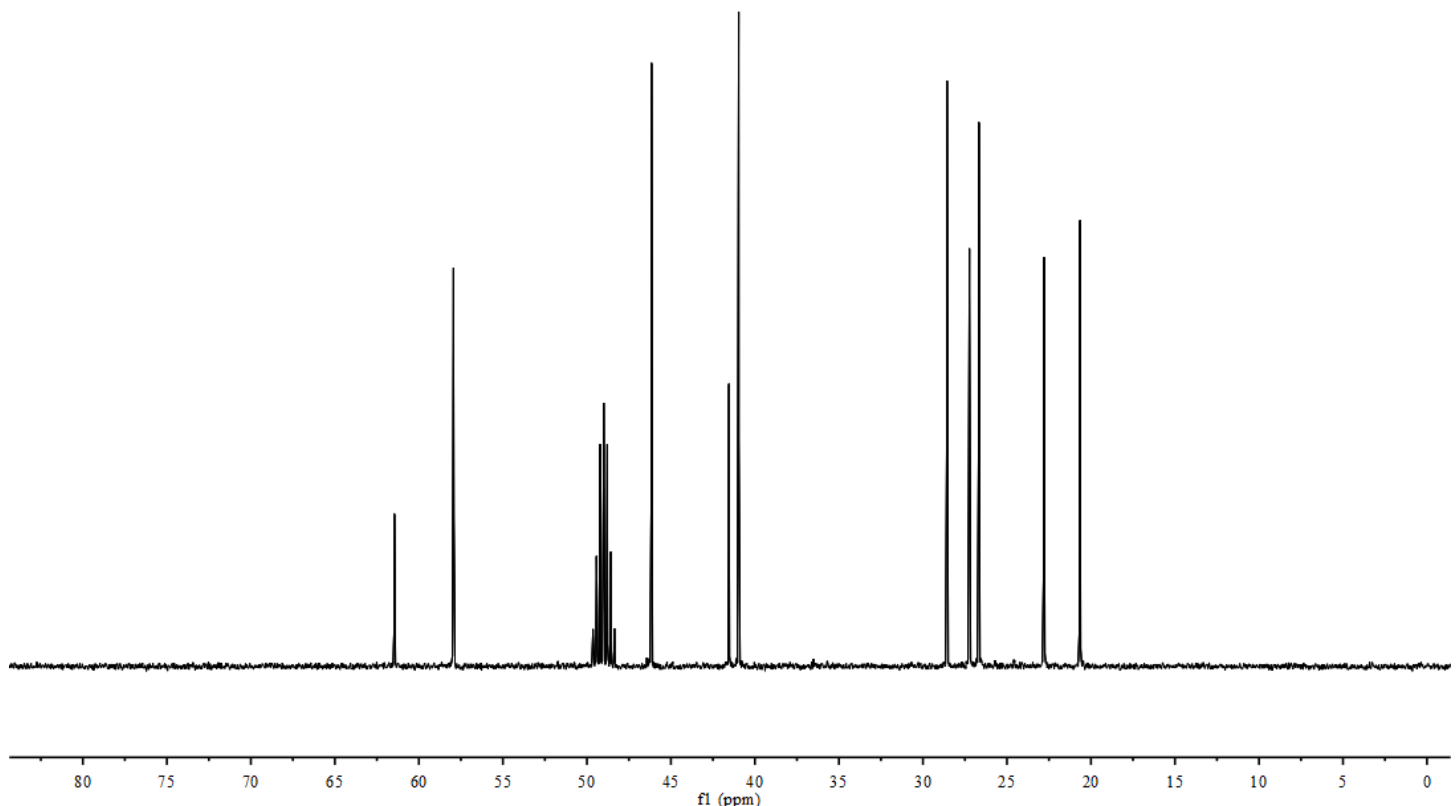

Figure S1. NMR spectra of compound 2a ( $\alpha$-pinene oxide): (a) ${ }^{1} \mathrm{H}$ NMR and (b) ${ }^{13} \mathrm{C}$ NMR. 
(a)

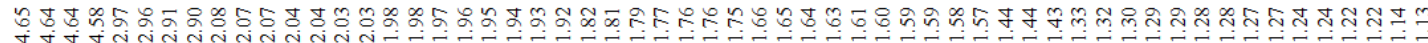<smiles>C=C(C)C1CCC2(C)OC2C1</smiles>

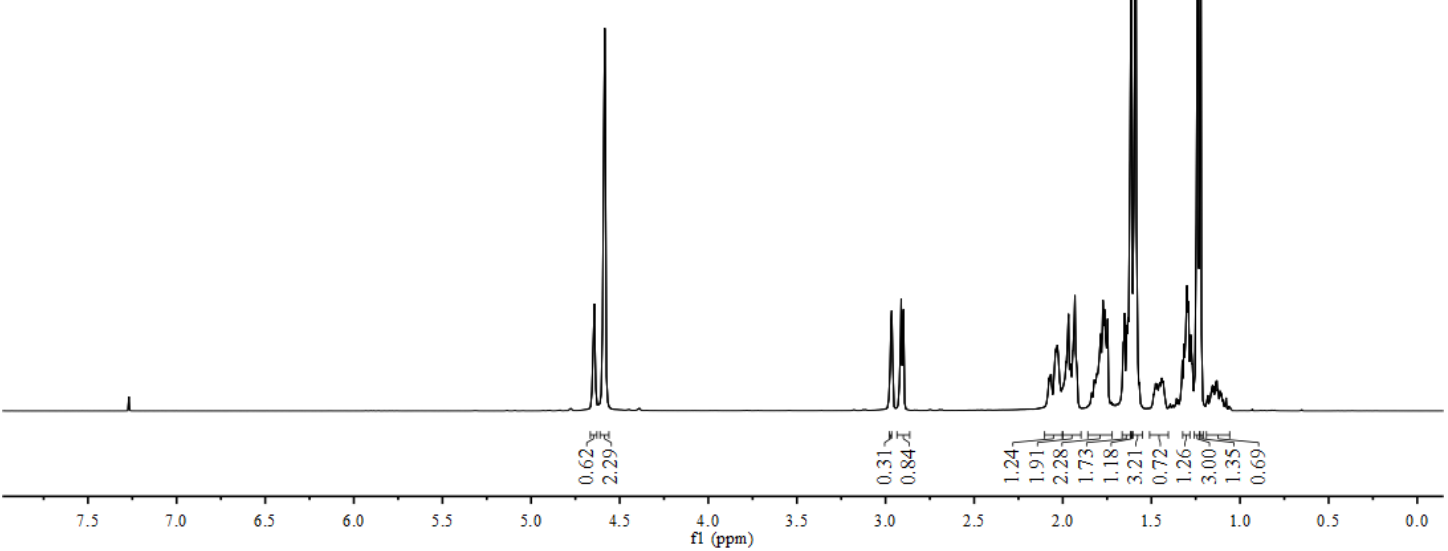

(b)

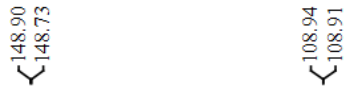

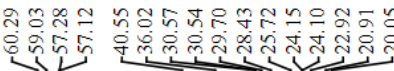
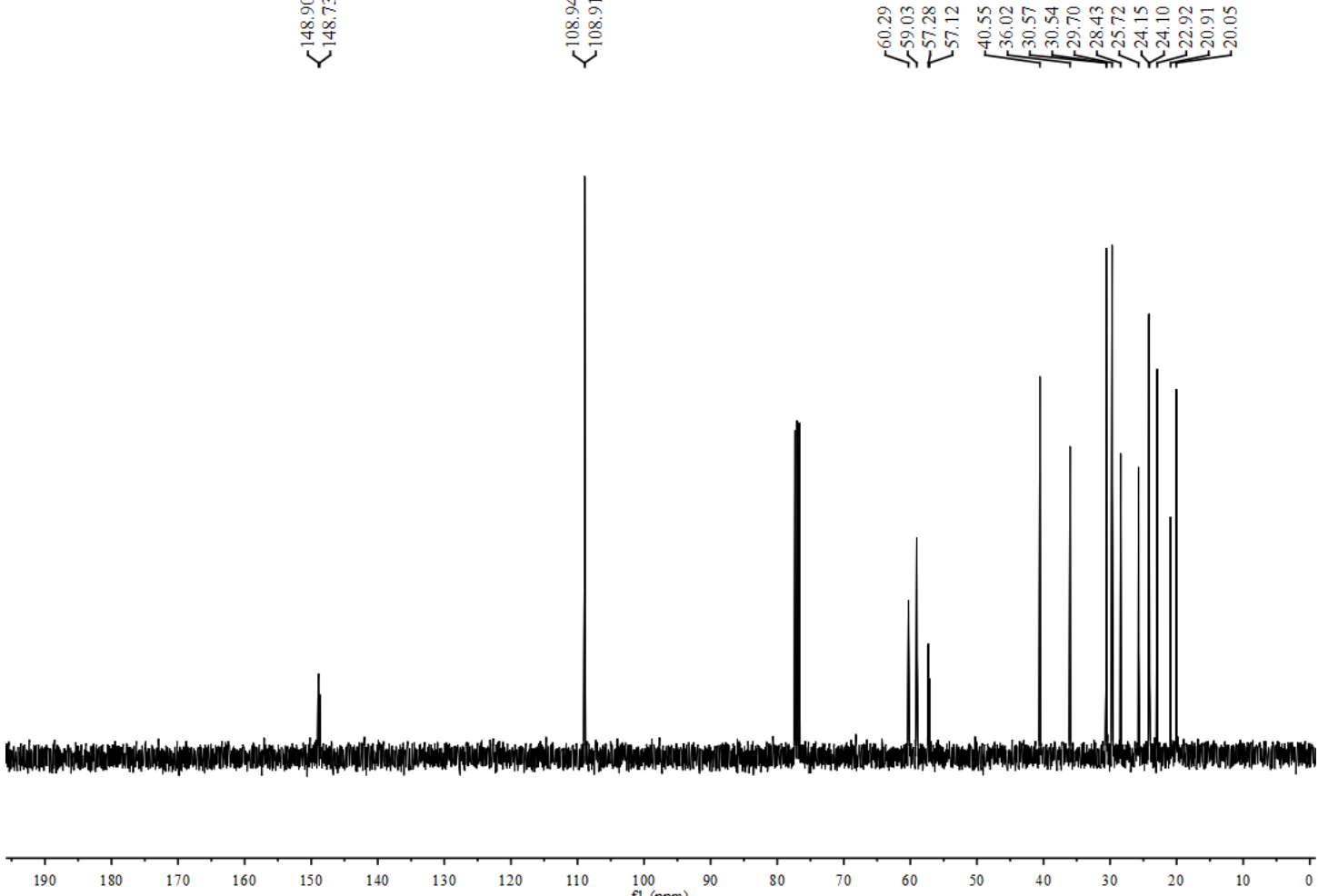

Figure S2. NMR spectra of compound 2b (limonene oxide): (a) ${ }^{1} \mathrm{H}$ NMR and (b) ${ }^{13} \mathrm{C}$ NMR. 
(a)

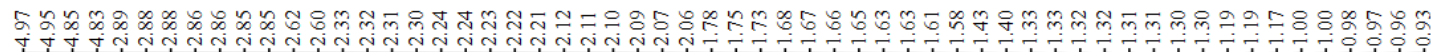

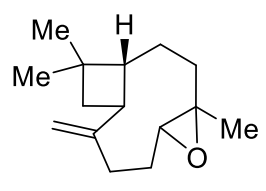

$2 c$

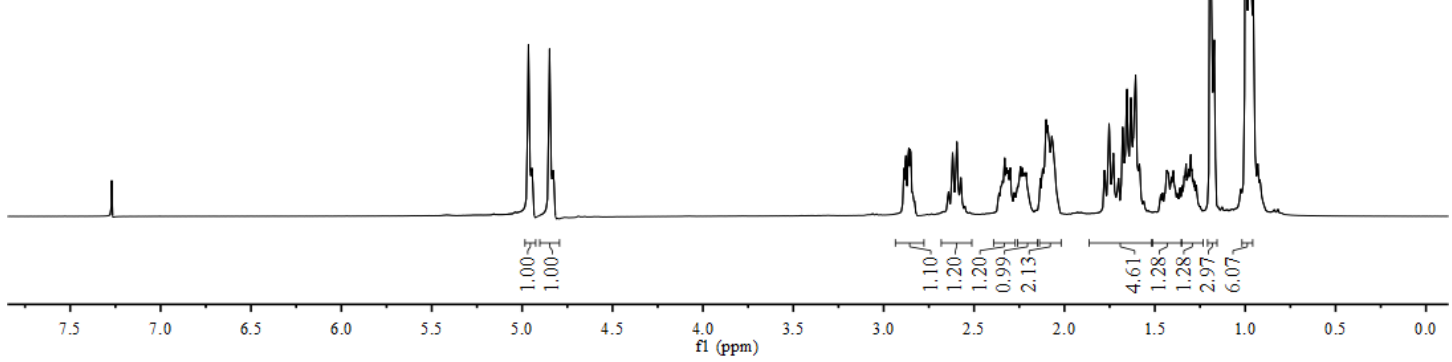

(b)

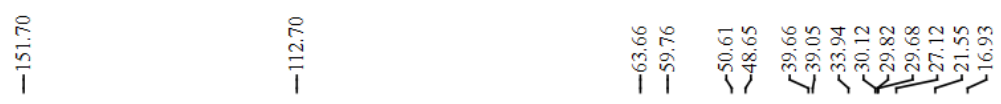
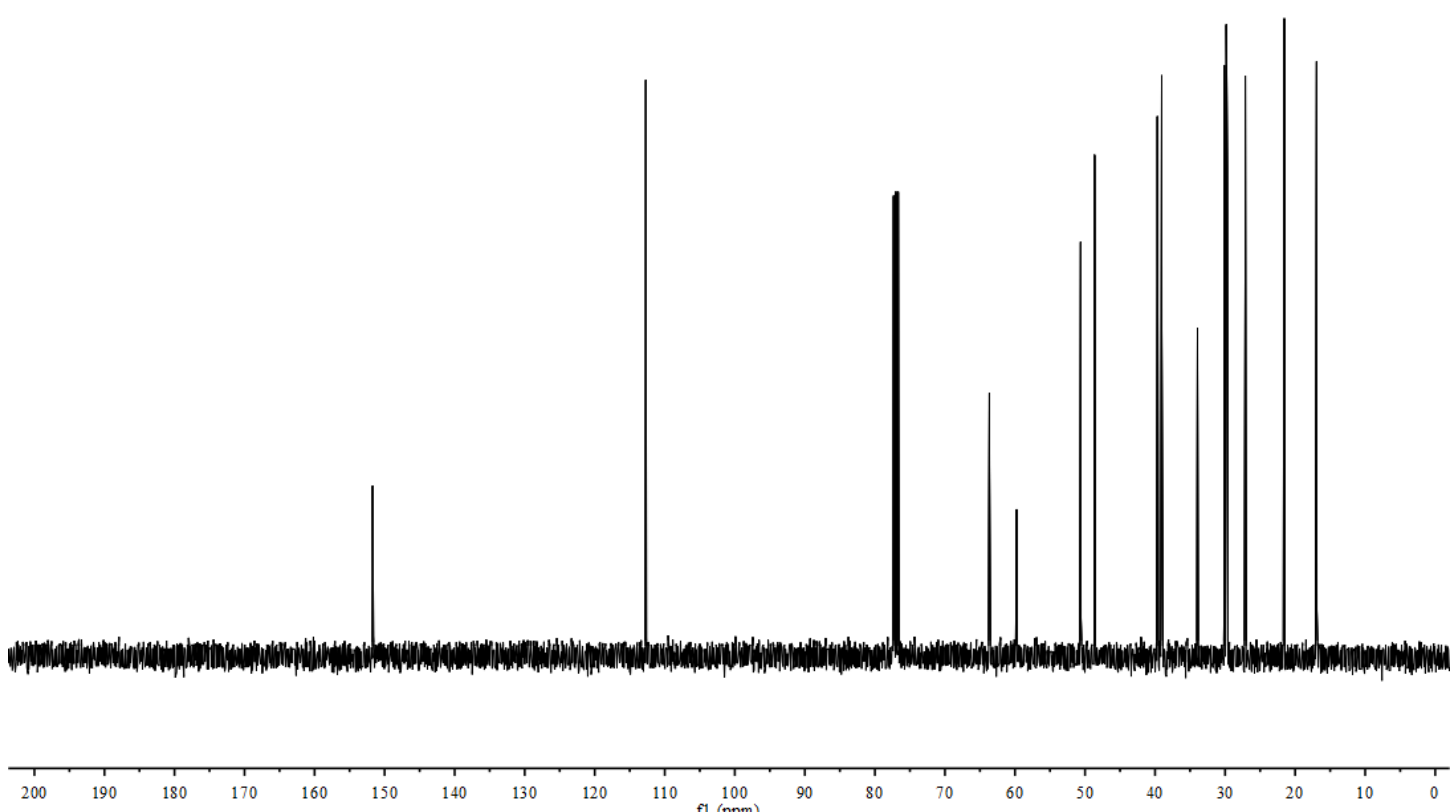

Figure S3. NMR spectra of of compound 2c ( $\beta$-caryophyllene oxide): (a) ${ }^{1} \mathrm{H}$ NMR and (b) ${ }^{13} \mathrm{C}$ NMR. 
(a)
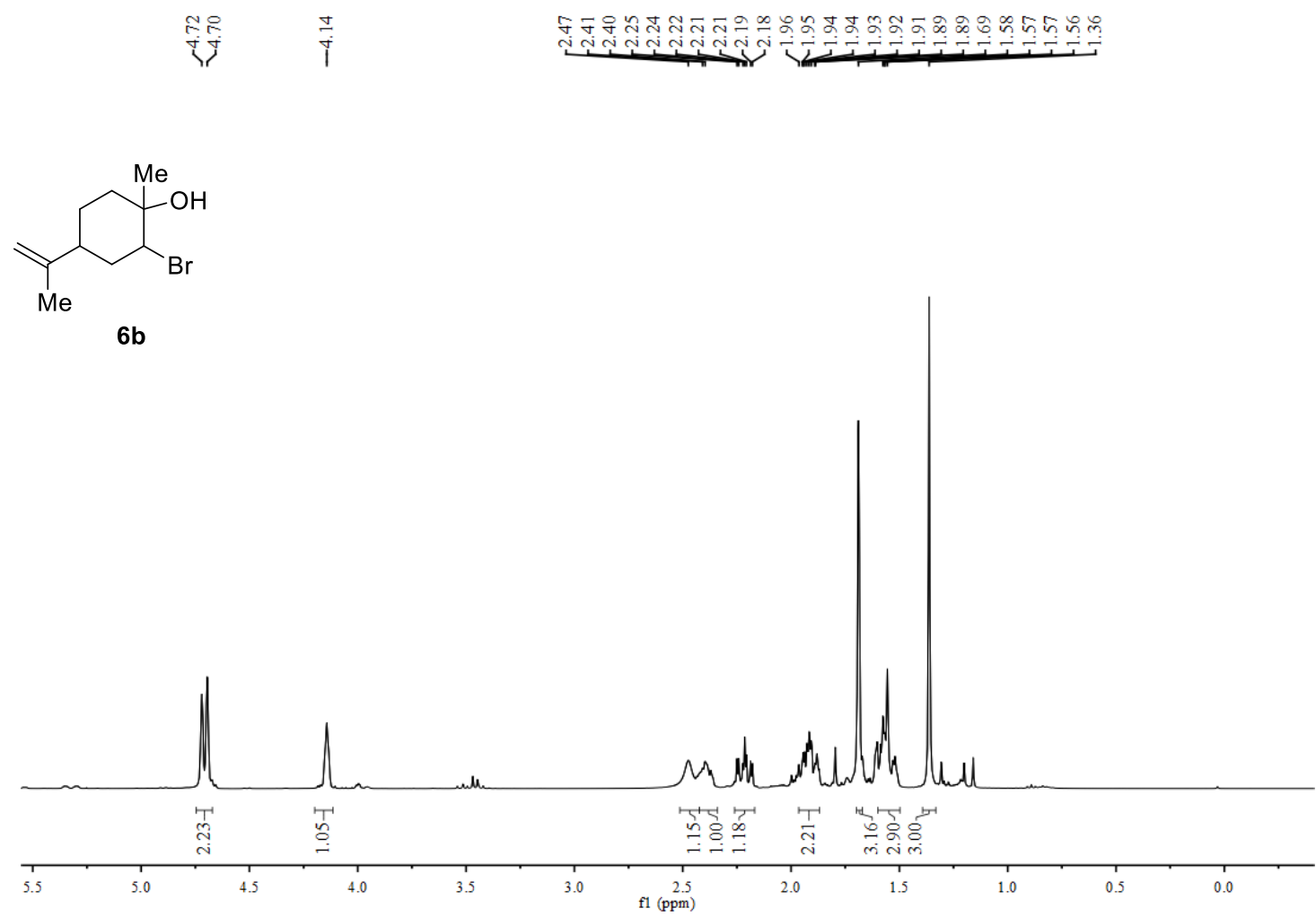

(b)
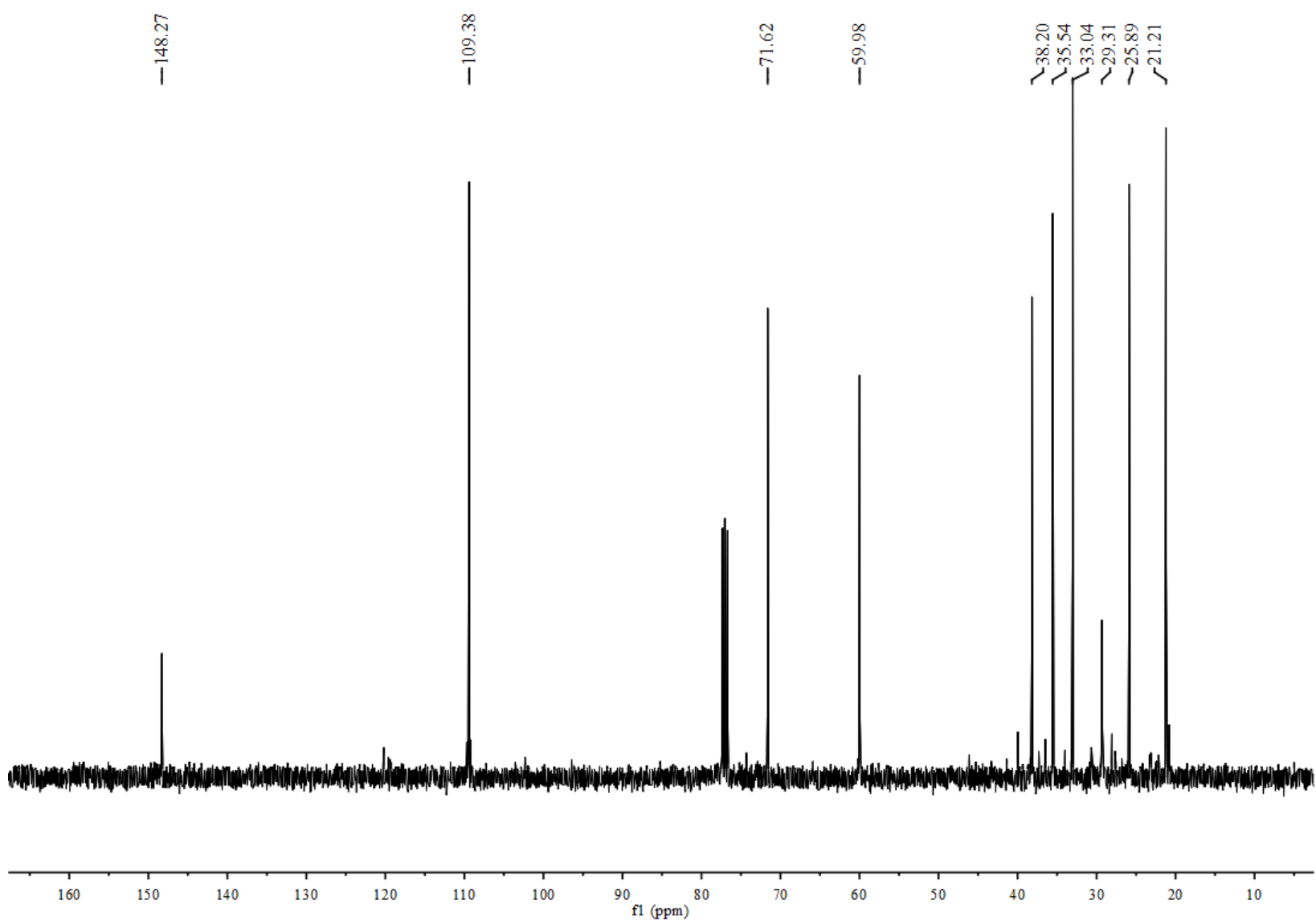
(c)

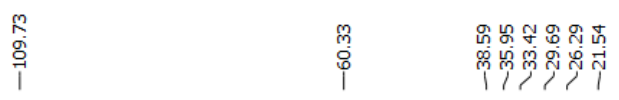

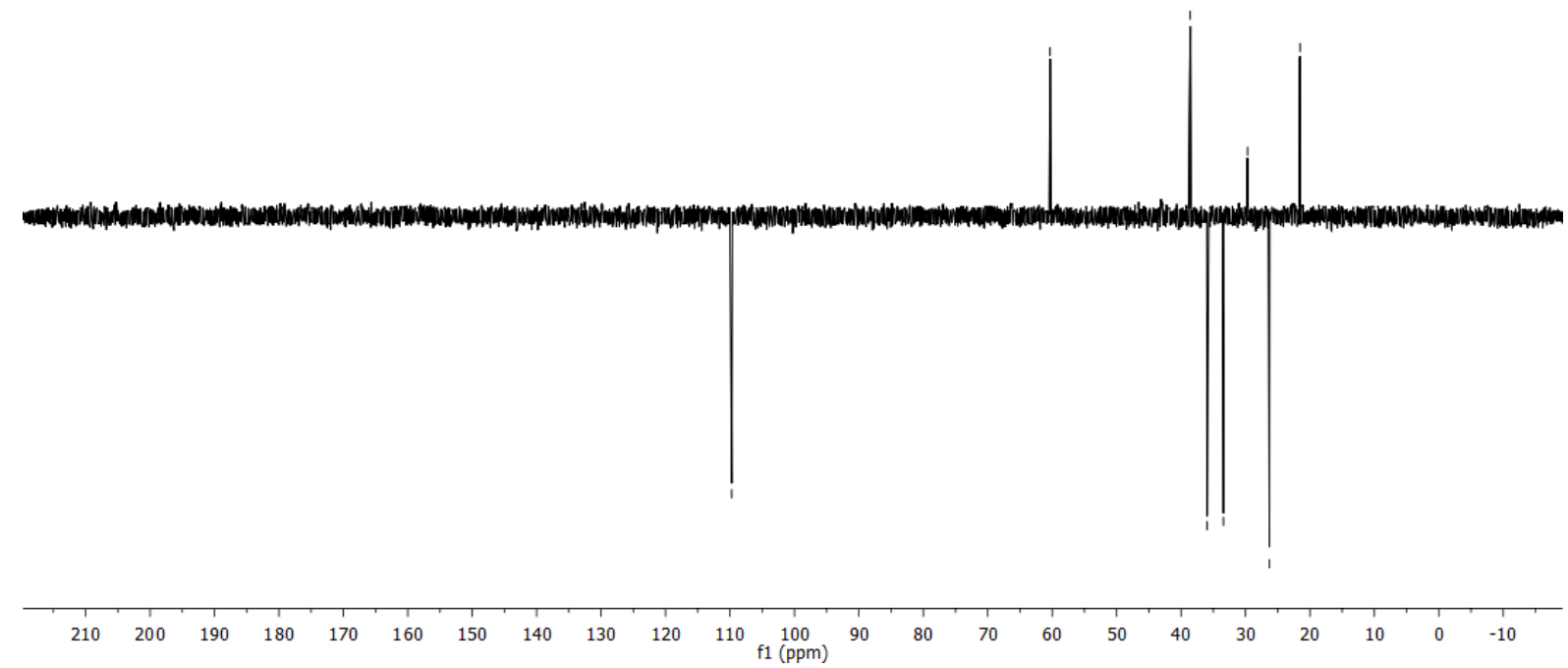

Figure S4. NMR spectra of compound $6 \mathbf{b}$ (2-bromo-1-methyl-4-(prop-1-en-2-yl)-cyclohexanol): (a) ${ }^{1} \mathrm{H}$ NMR, (b) ${ }^{13} \mathrm{C}$ NMR, and (c) DEPT-135. 
(a)

$\stackrel{\infty}{i}$
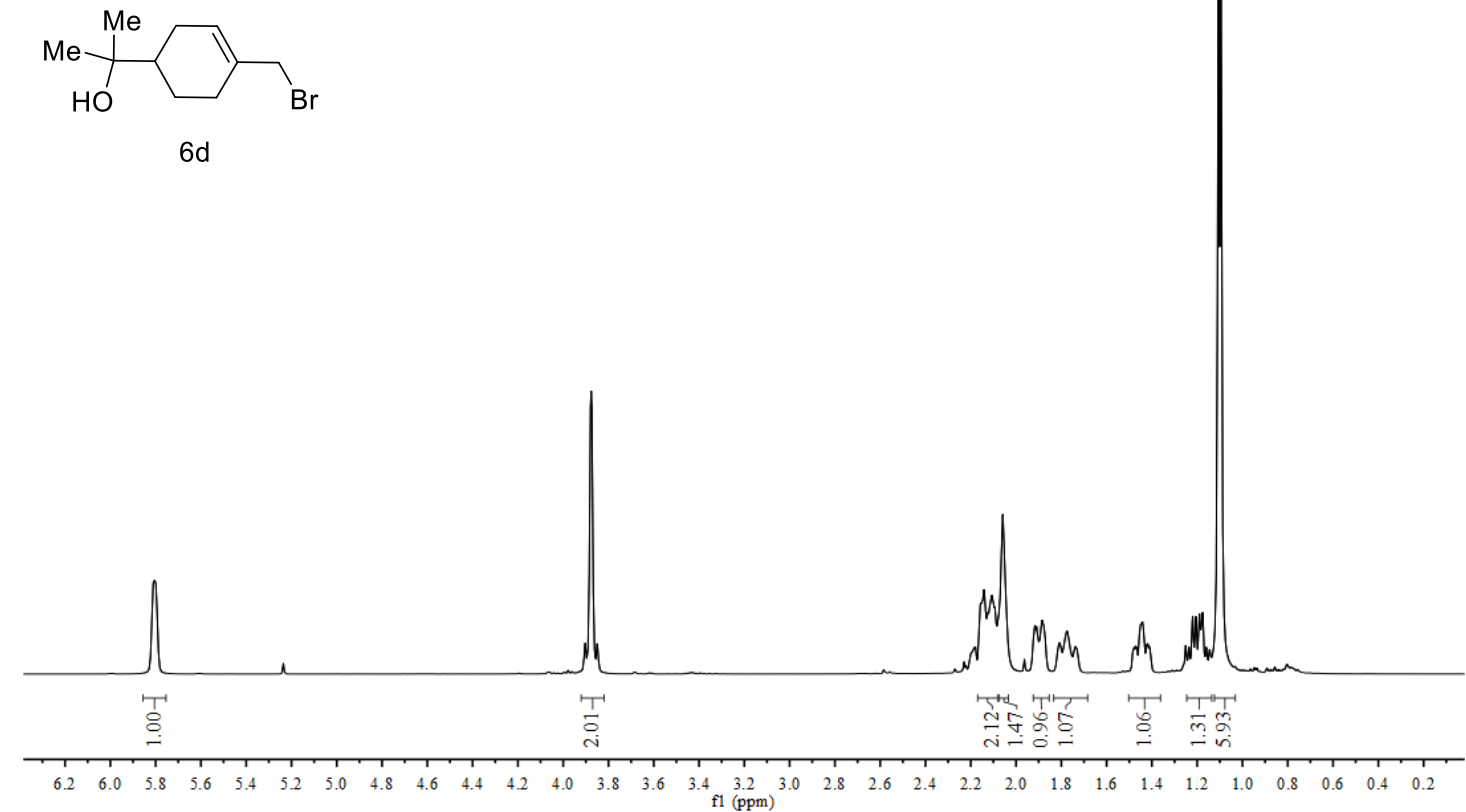

(b)

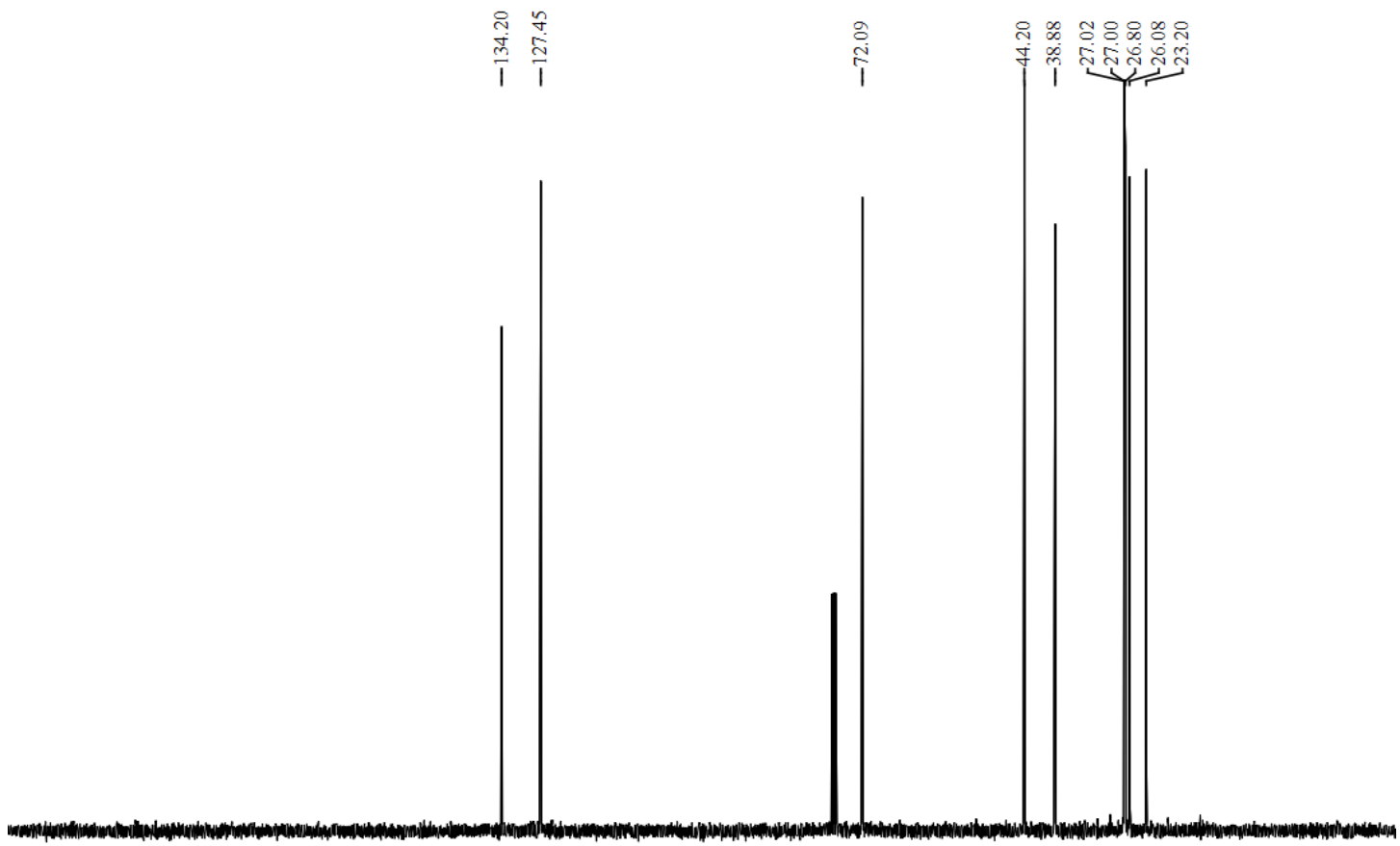

$\begin{array}{lllllllllllllllllllllllllllllll}210 & 200 & 190 & 180 & 170 & 160 & 150 & 140 & 130 & 120 & 110 & 100 & 90 & 80 & 70 & 60 & 50 & 40 & 30 & 20 & 10 & 0 & -10 & 10\end{array}$ 
(c)

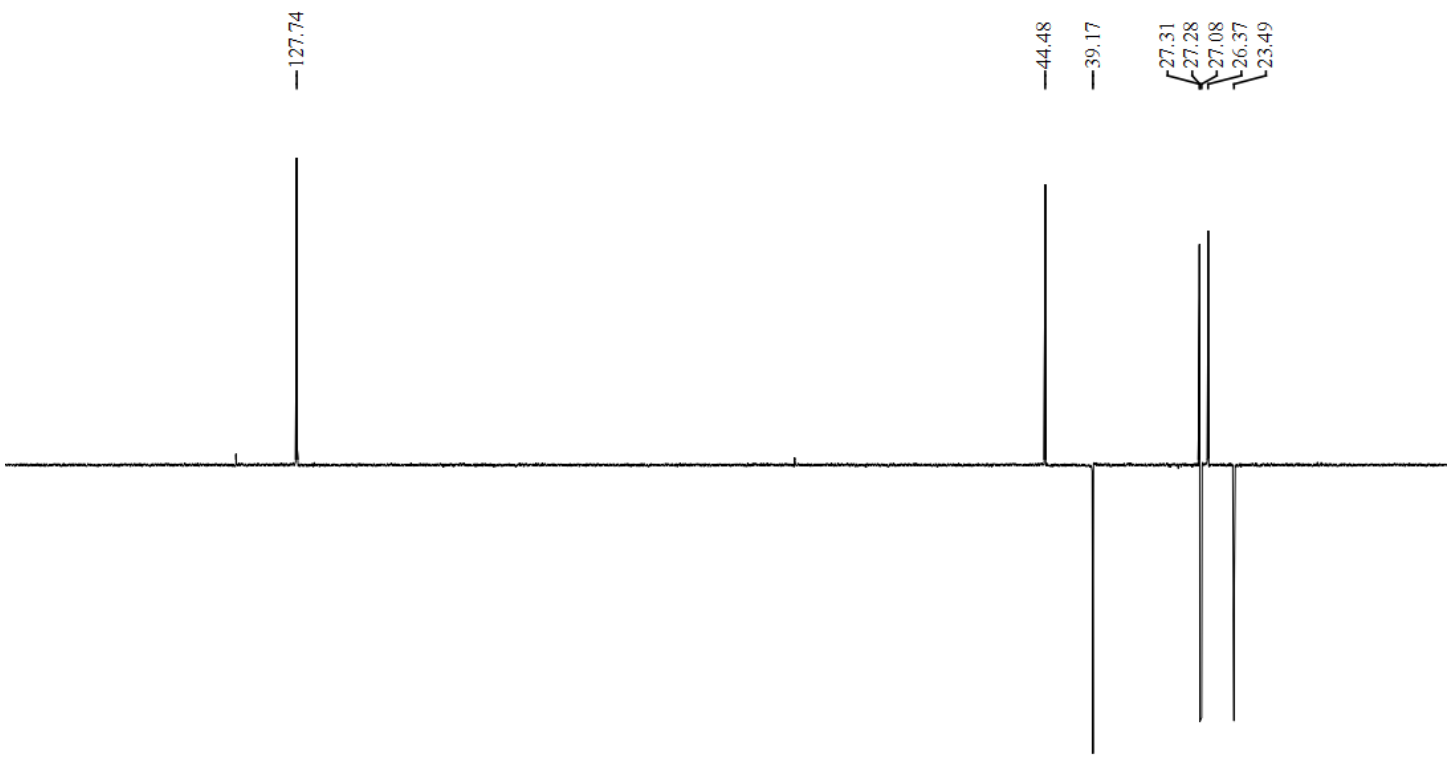

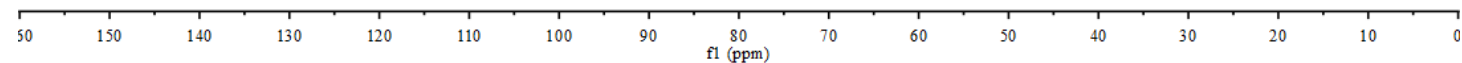

Figure S5. NMR spectra of compound 6d (2-(4-(bromomethyl)cyclohex-3-en-1-yl)propan-2-ol): (a) ${ }^{1} \mathrm{H}$ NMR, (b) ${ }^{13} \mathrm{C}$ NMR, and (c) DEPT-135. 
(a)

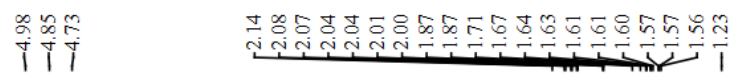<smiles>C=C(C)C1CCC(O)(O)C(O[N+](=O)[O-])C1</smiles>

$3 b^{\prime}$

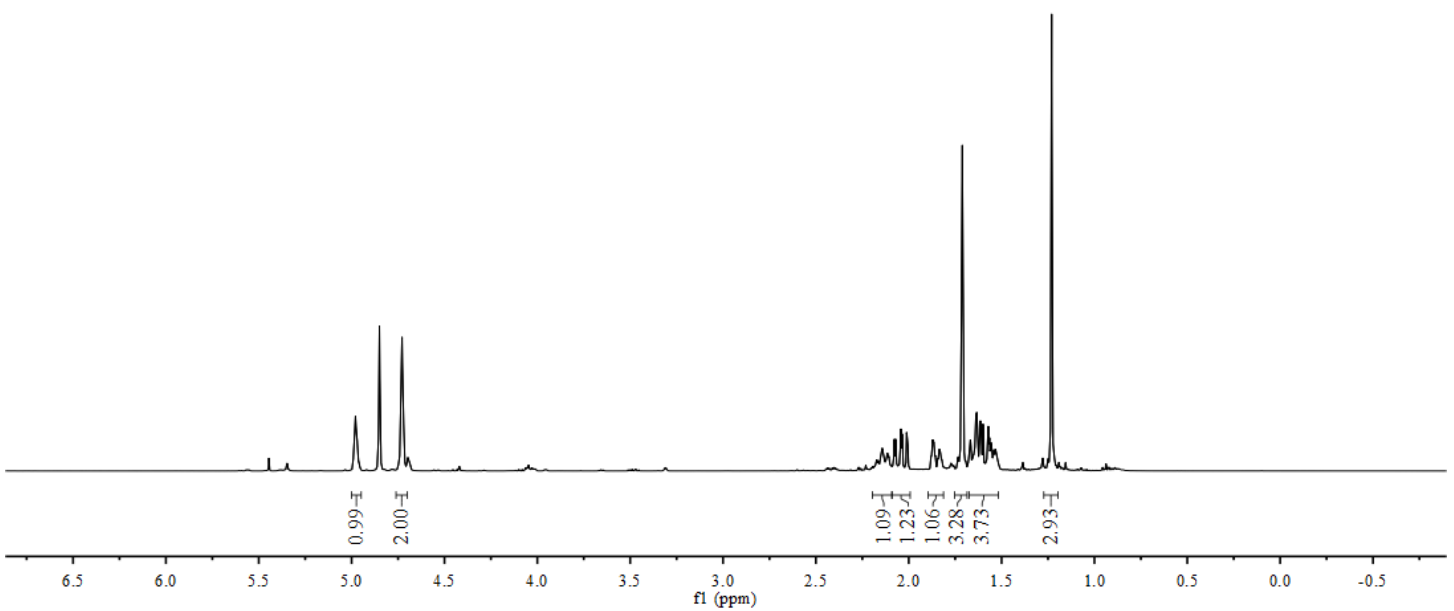

(b)

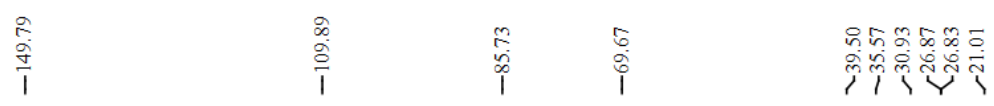

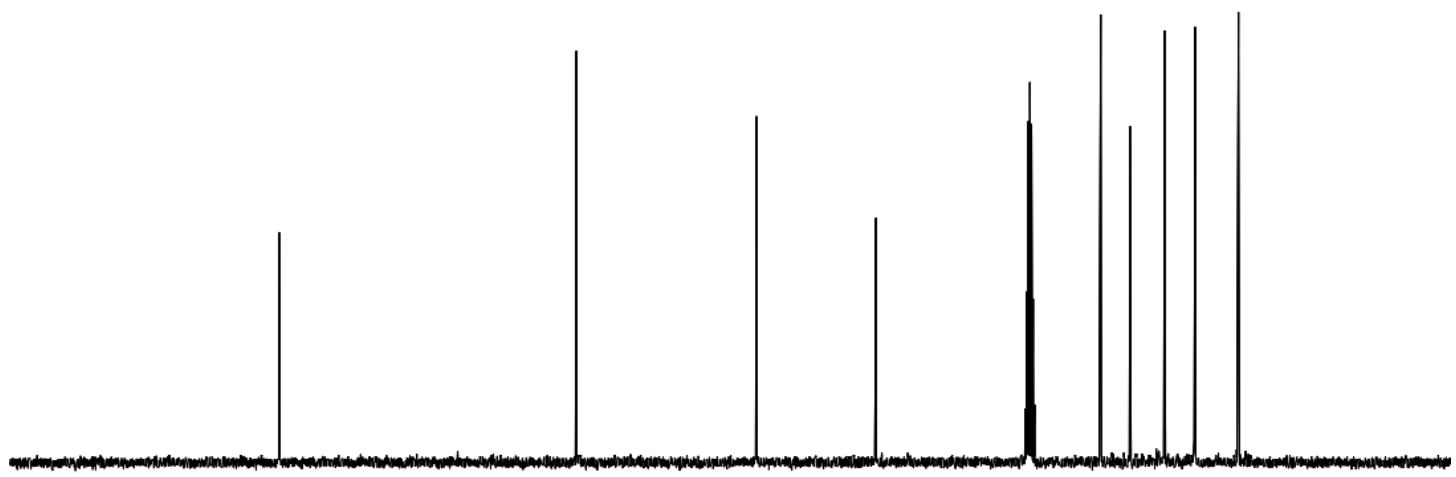


(c)

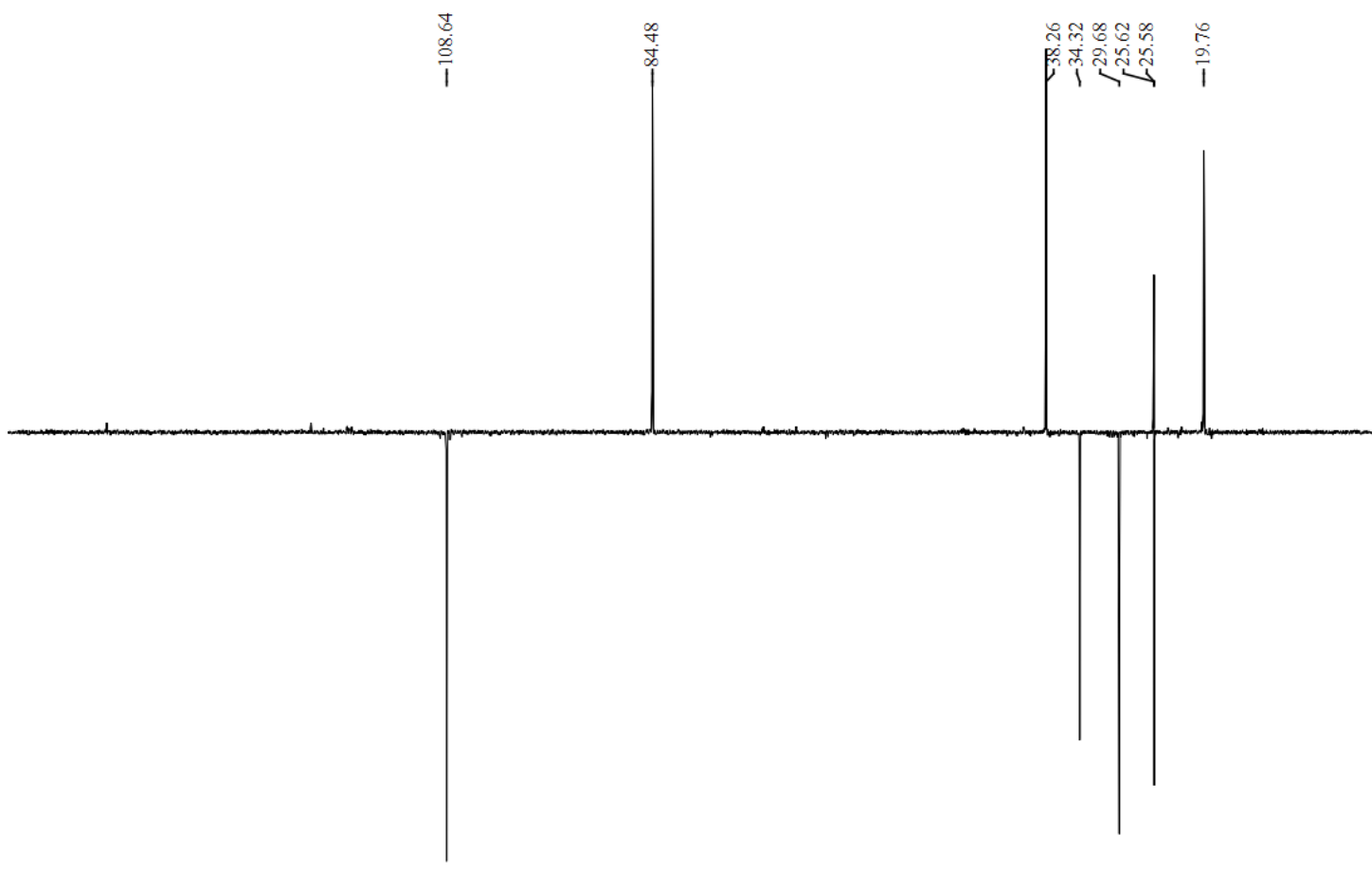

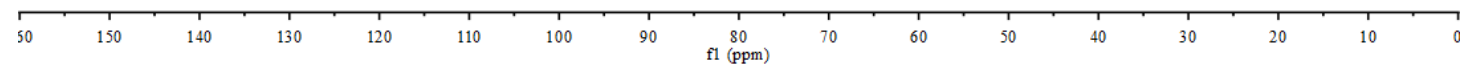

Figure S6. NMR spectra of compound 3b' (2-hydroxy-2-methyl-5-(prop-1-en-2-yl)-cyclohexyl nitrate): (a) ${ }^{1} \mathrm{H}$ NMR, (b) ${ }^{13} \mathrm{C}$ NMR, and (c) DEPT- 135 . 
(a)

\section{iूa

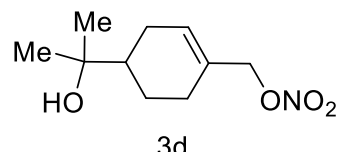

$3 d$

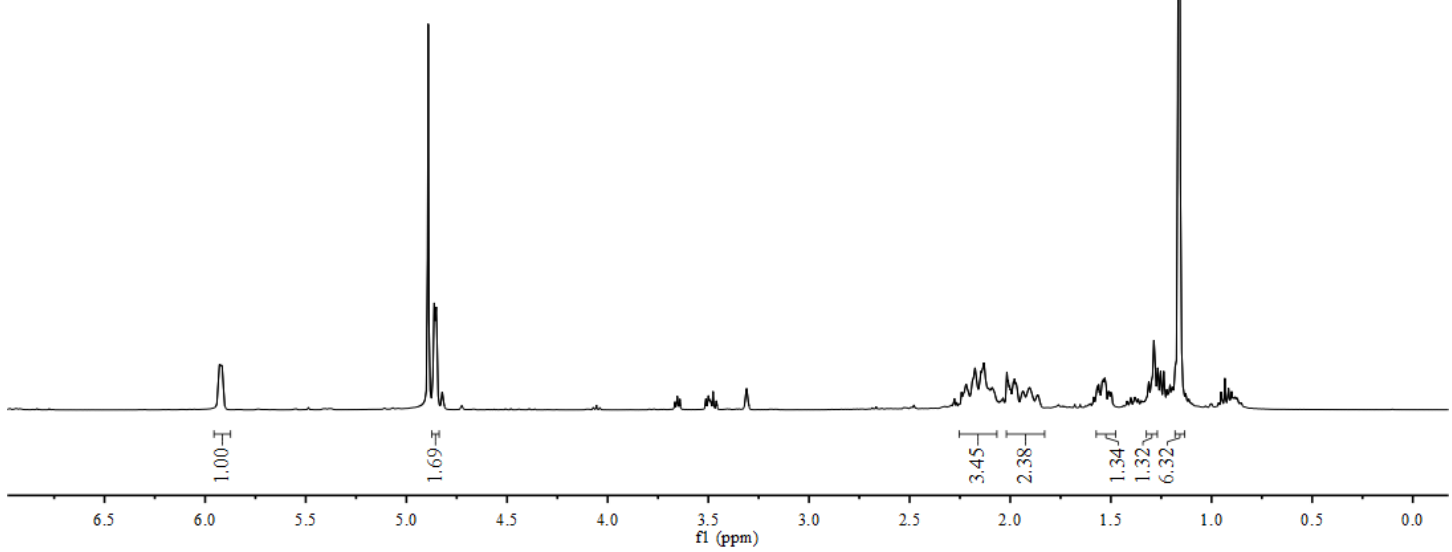

(b)

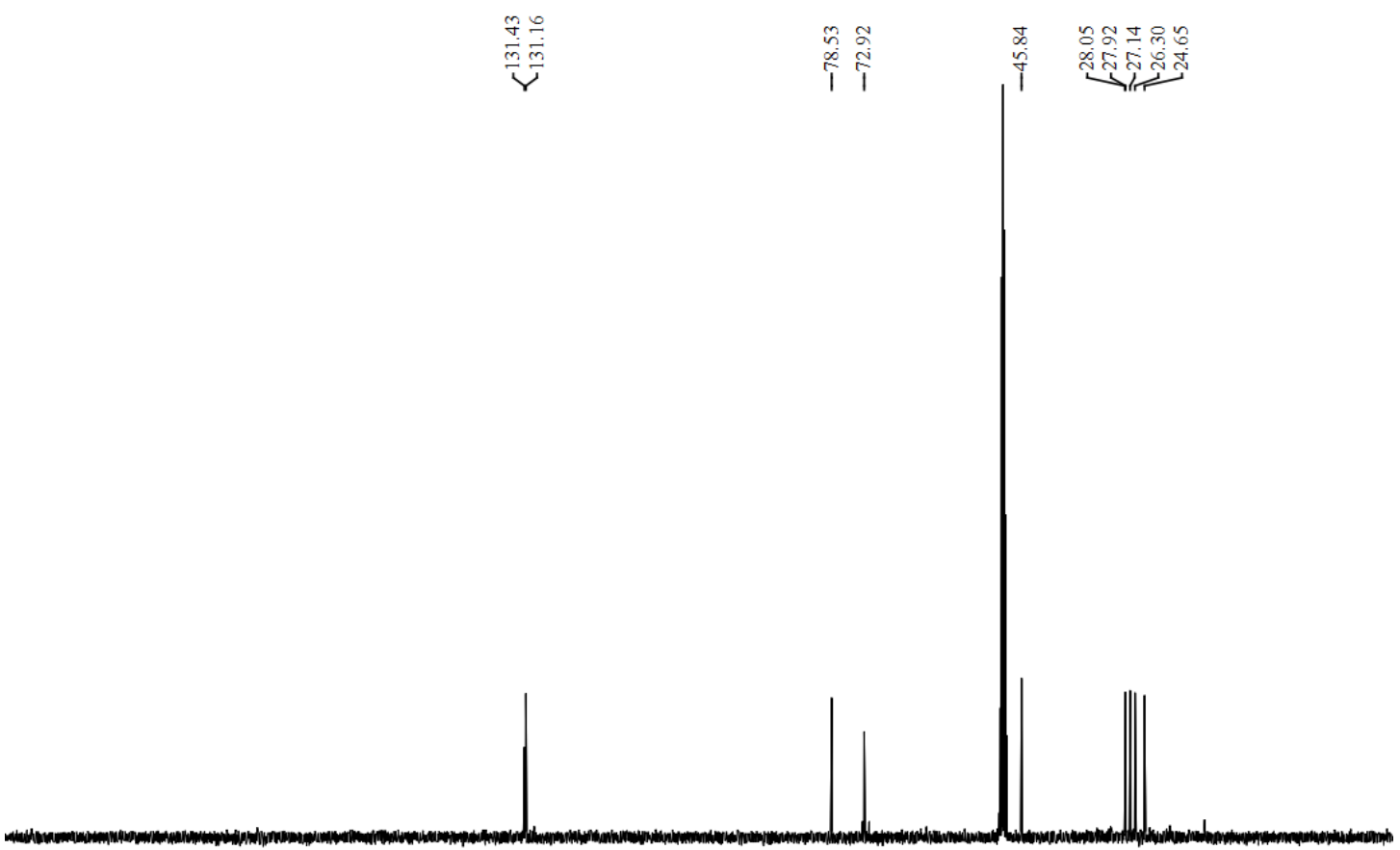

$\begin{array}{llllllllllllllllllllllllllllllllll}20 & 210 & 200 & 190 & 180 & 170 & 160 & 150 & 140 & 130 & 120 & 110 & 100 & 90 & 80 & 70 & 60 & 50 & 40 & 30 & 20 & 10 & 0 & -10\end{array}$ 
(c)

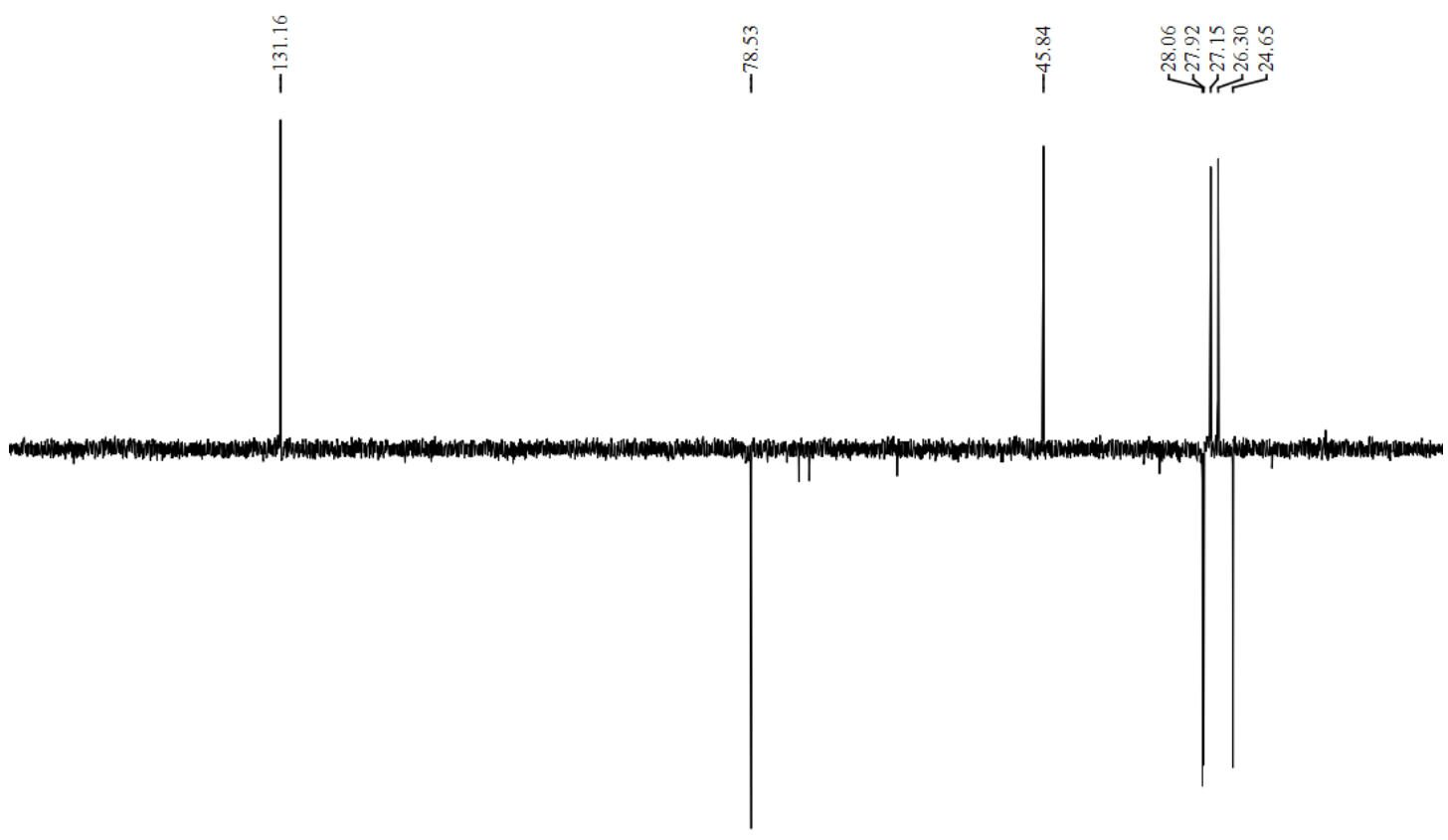

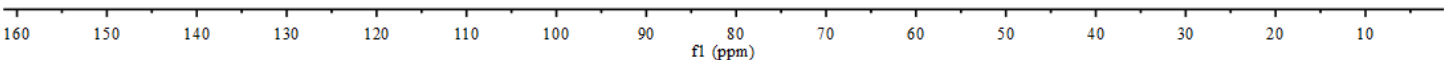

Figure S7. NMR spectra of compound 3d ((4-(2-hydroxypropan-2-yl) cyclohex-1-en-1-yl) -methyl nitrate): (a) ${ }^{1} \mathrm{H}$ NMR, (b) ${ }^{13} \mathrm{C}$ NMR, and (c) DEPT-135. 
(a)

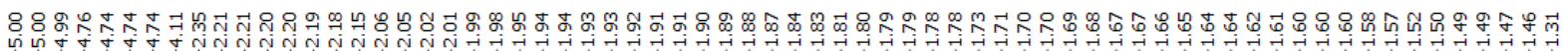<smiles>C=C(C)C1CCC(C)(O[N+]([O-])([O-])[O-])C(O)C1</smiles>

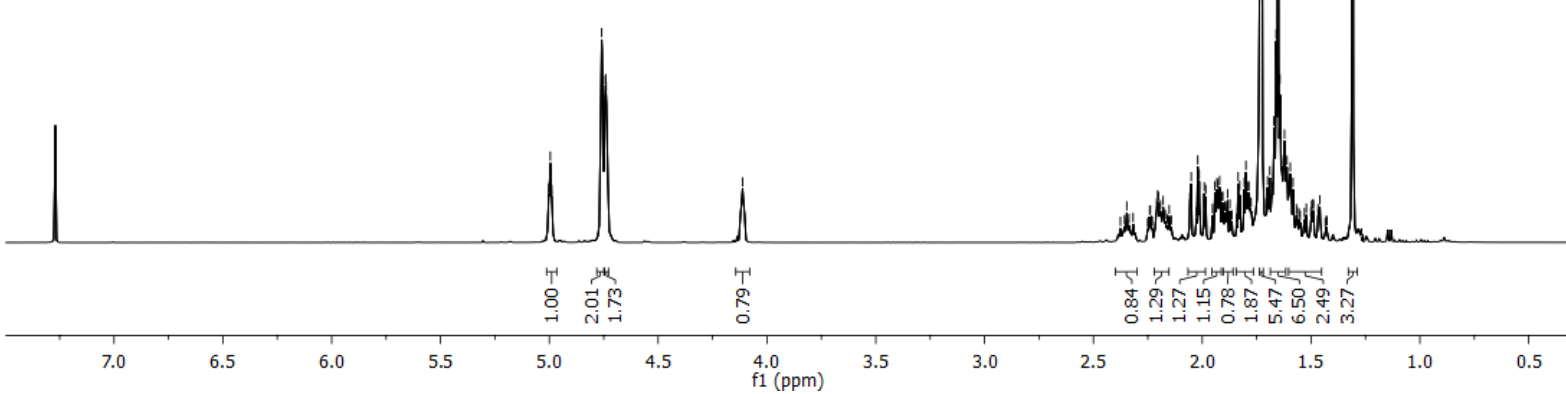

(b)

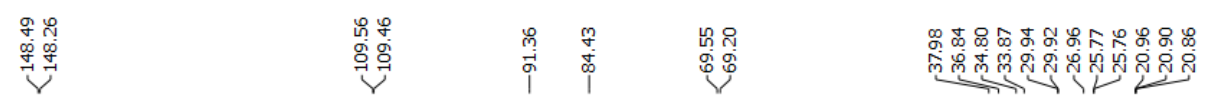

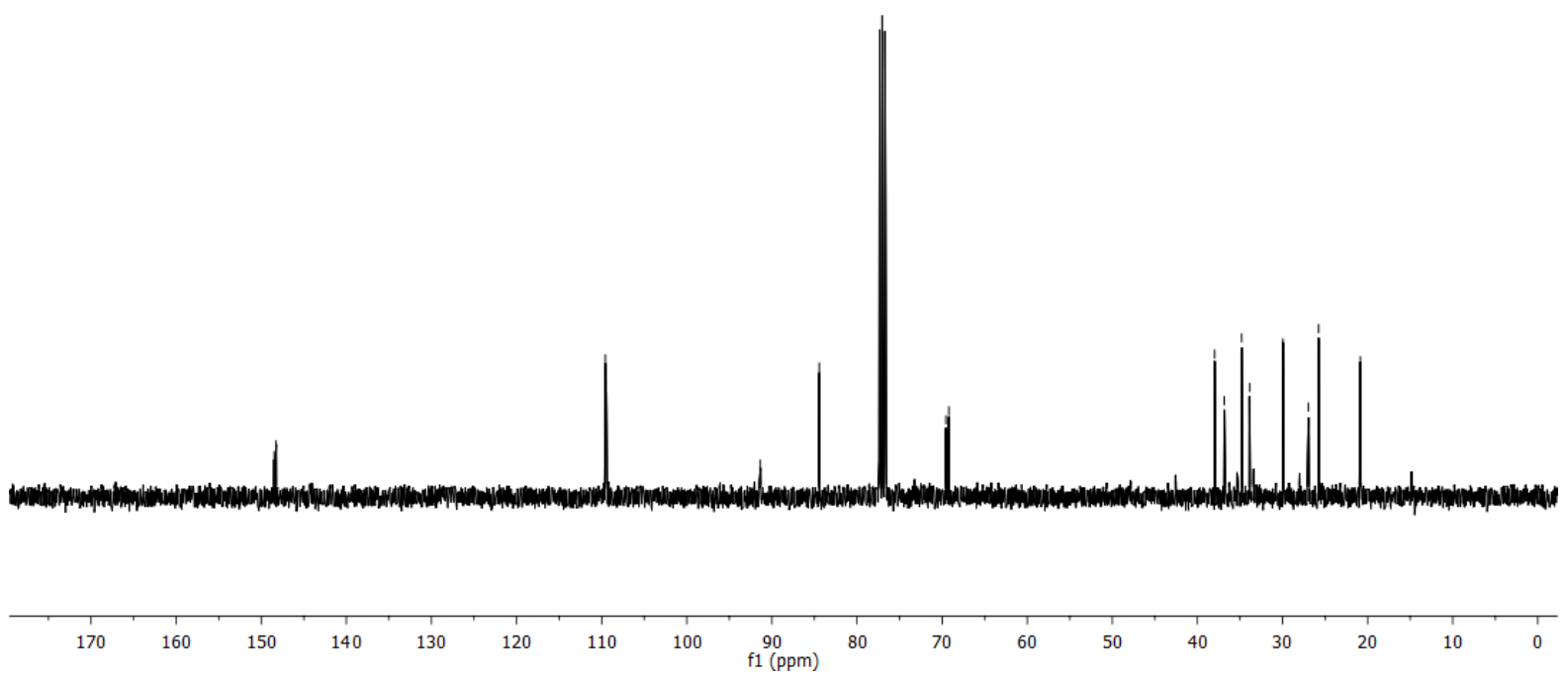


(c)

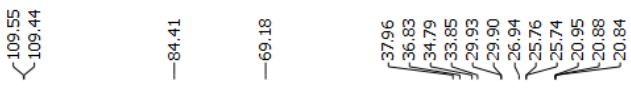

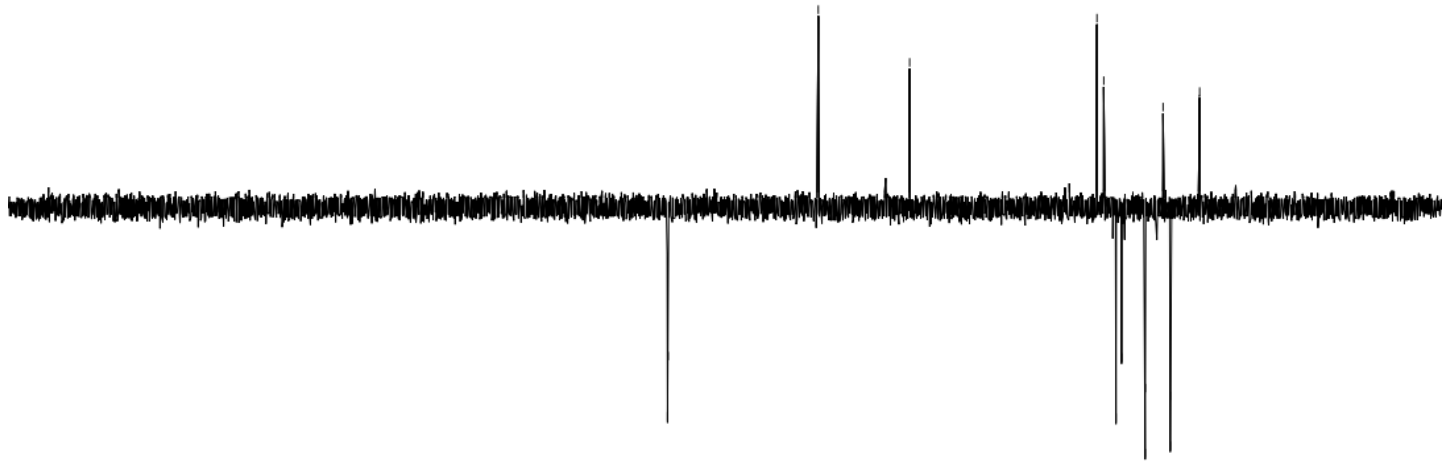

$\begin{array}{lllllllllllllllllllllll}210 & 200 & 190 & 180 & 170 & 160 & 150 & 140 & 130 & 120 & 110 & \underset{\mathrm{f} 1}{100}(\mathrm{ppm}) & 90 & 80 & 70 & 60 & 50 & 40 & 30 & 20 & 10 & 0 & -10\end{array}$

Figure S8. NMR spectra of compound 2-hydroxy-1-methyl-4-(prop-1-en-2-yl)cyclohexyl nitrate (3b) \& 2-hydroxy-2-methyl-5-(prop-1-en-2-yl)cyclohexyl nitrate (3b'): (a) ${ }^{1} \mathrm{H}$ NMR, (b) ${ }^{13} \mathrm{C}$ NMR, and (c) DEPT-135. 
(a)

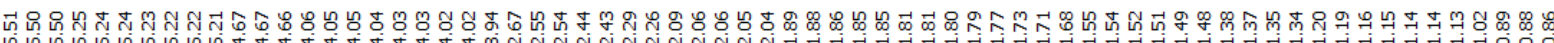
й<smiles>C=C(C)C1CC=C(C)C(O)C1</smiles>

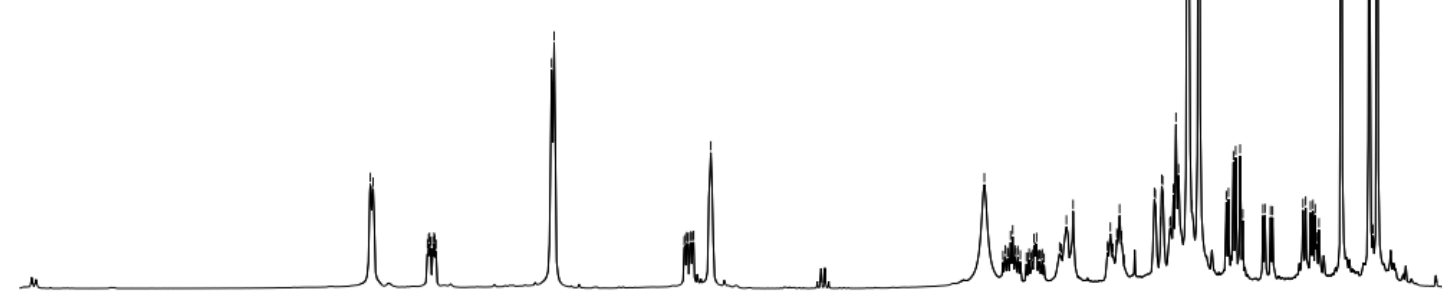

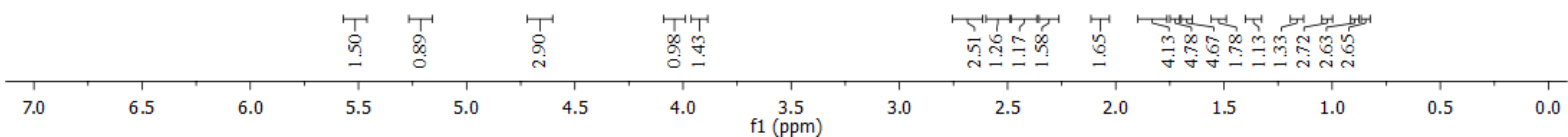

(b)

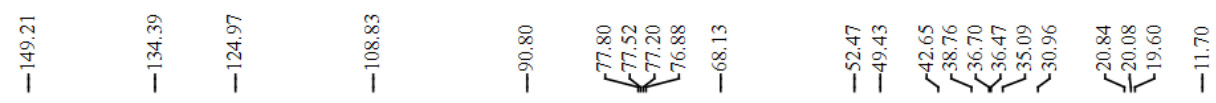
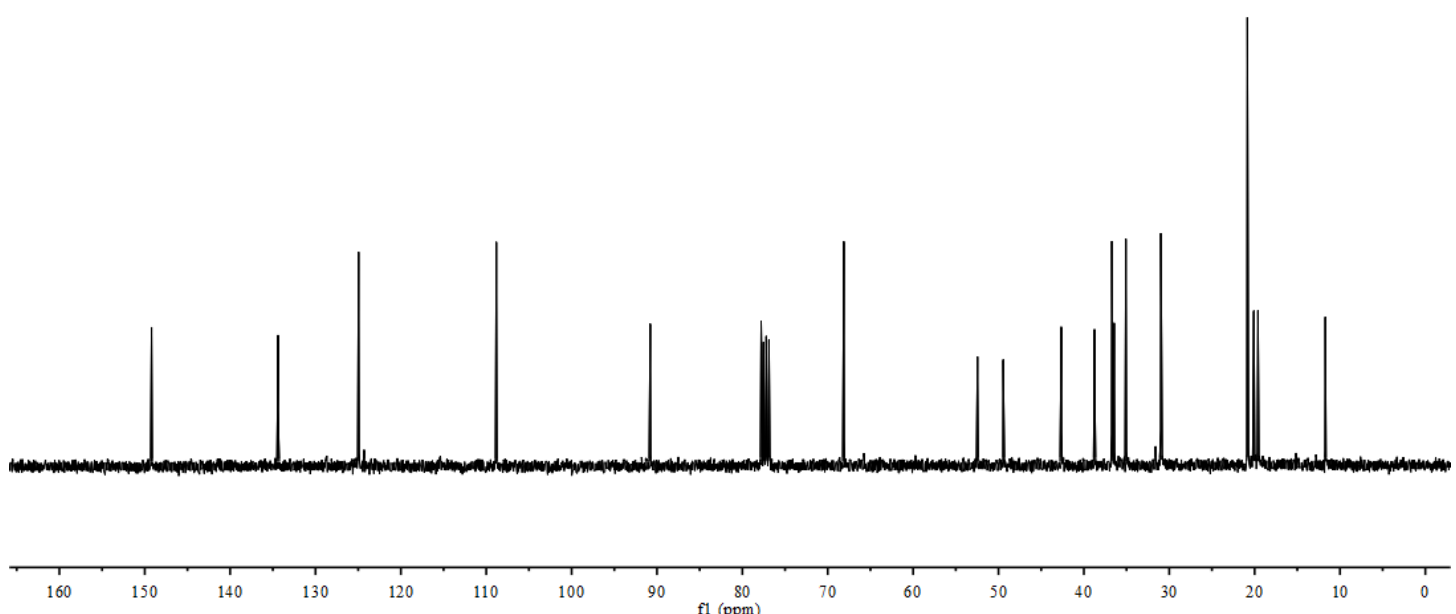
(c)

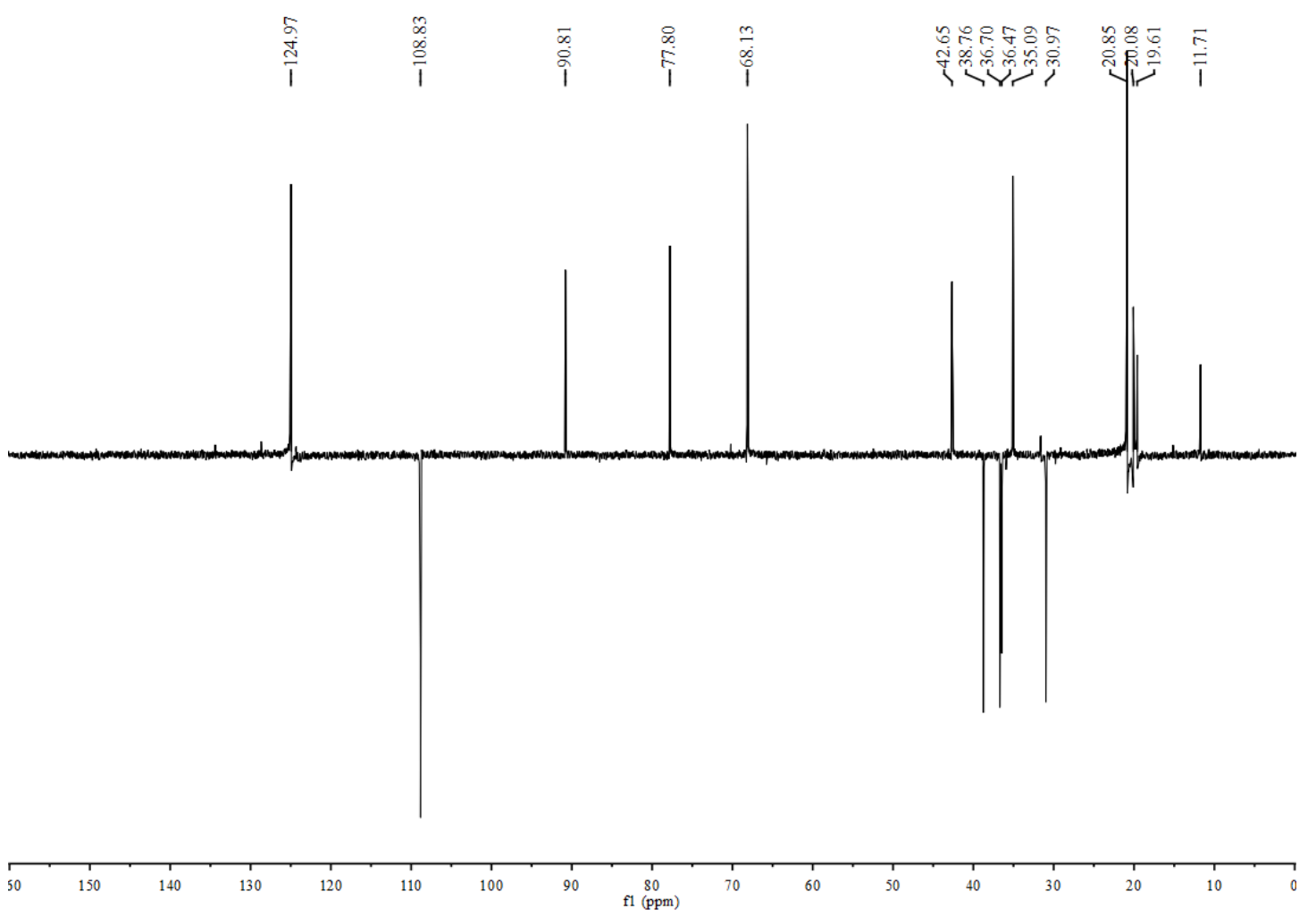

Figure S9. NMR spectra of mixture 3a' \& Carveol ((6-hydroxy-1,7,7-trimethylbicyclo[2.2.1] -heptan2-yl nitrate \& 2-methyl-5-(prop-1-en-2-yl)cyclohex-2-enol): (a) ${ }^{1} \mathrm{H}$ NMR, (b) ${ }^{13} \mathrm{C}$ NMR, and (c) DEPT135. 
(a)

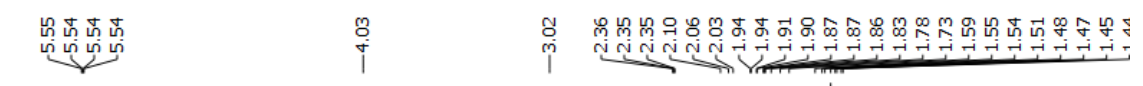
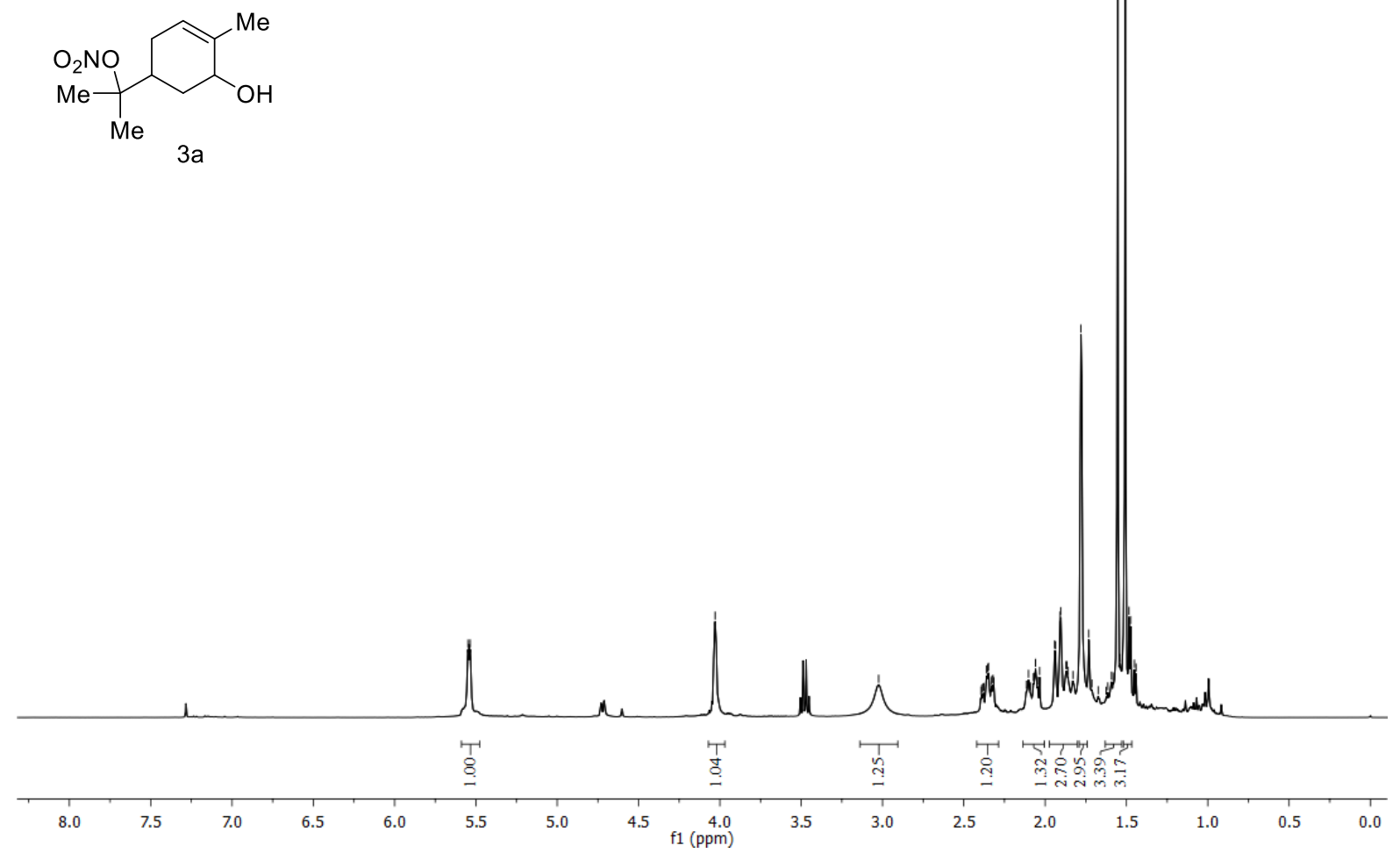

(b)

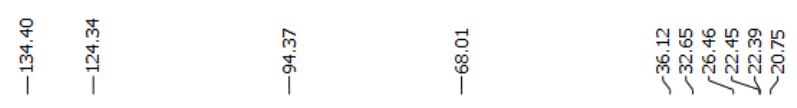

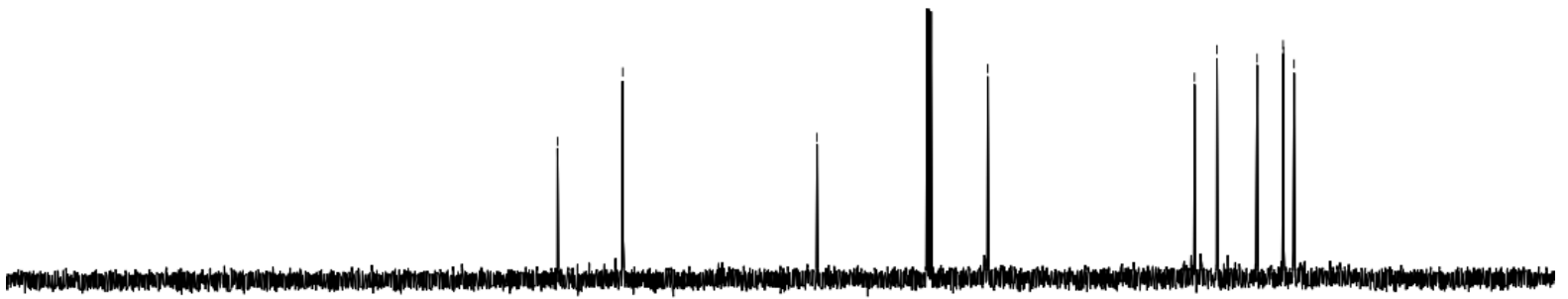

$\begin{array}{lllllllllllllllllllllllll}210 & 200 & 190 & 180 & 170 & 160 & 150 & 140 & 130 & 120 & 110 & 100 & 90 & 80 & 70 & 60 & 50 & 40 & 30 & 20 & 10 & 0 & -10\end{array}$ 
(c)

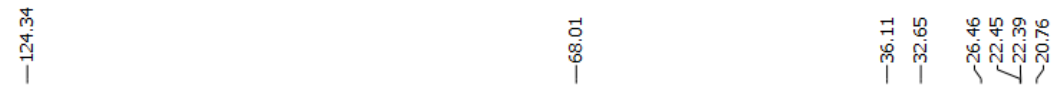

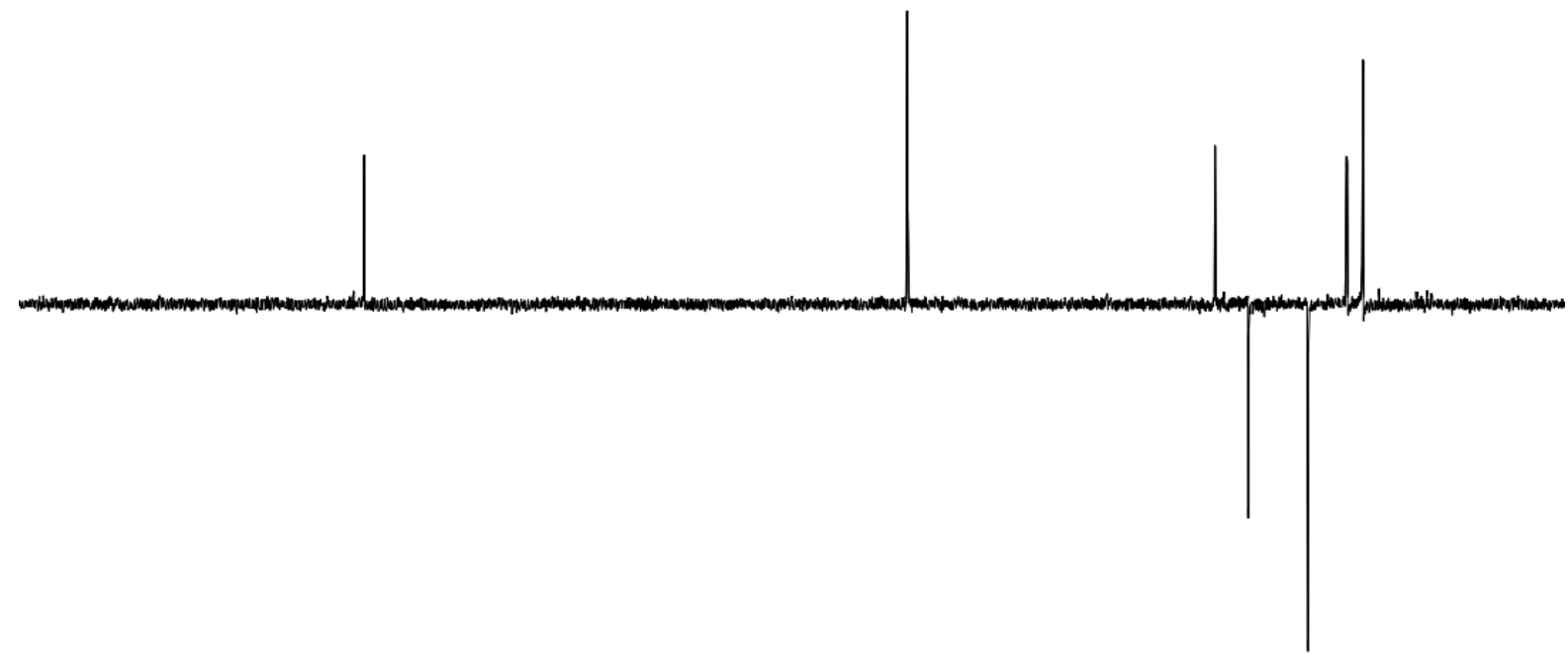

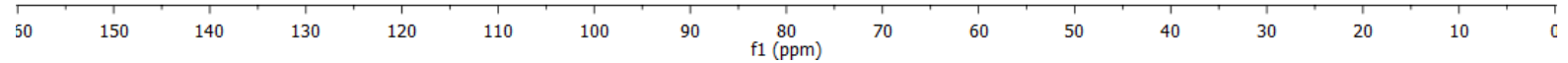

Figure S10. NMR spectra of compound 3a (2-(5-hydroxy-4-methylcyclohex-3-en-1-yl)-propan-2-yl nitrate): (a) ${ }^{1} \mathrm{H}$ NMR, (b) ${ }^{13} \mathrm{C}$ NMR, and (c) DEPT-135. 
(a)

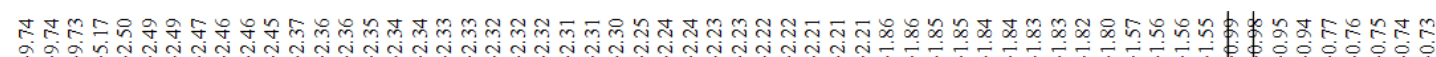<smiles>CC1=CCC(CC(=O)F)C1(C)C</smiles>

Campholenaldehyde

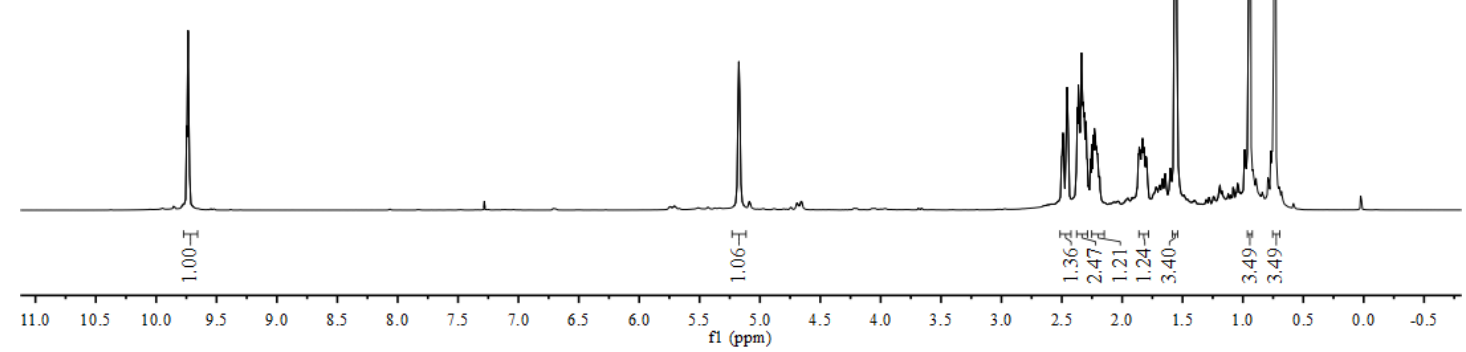

(b)
离

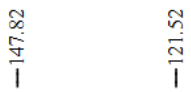

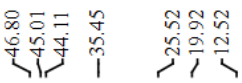

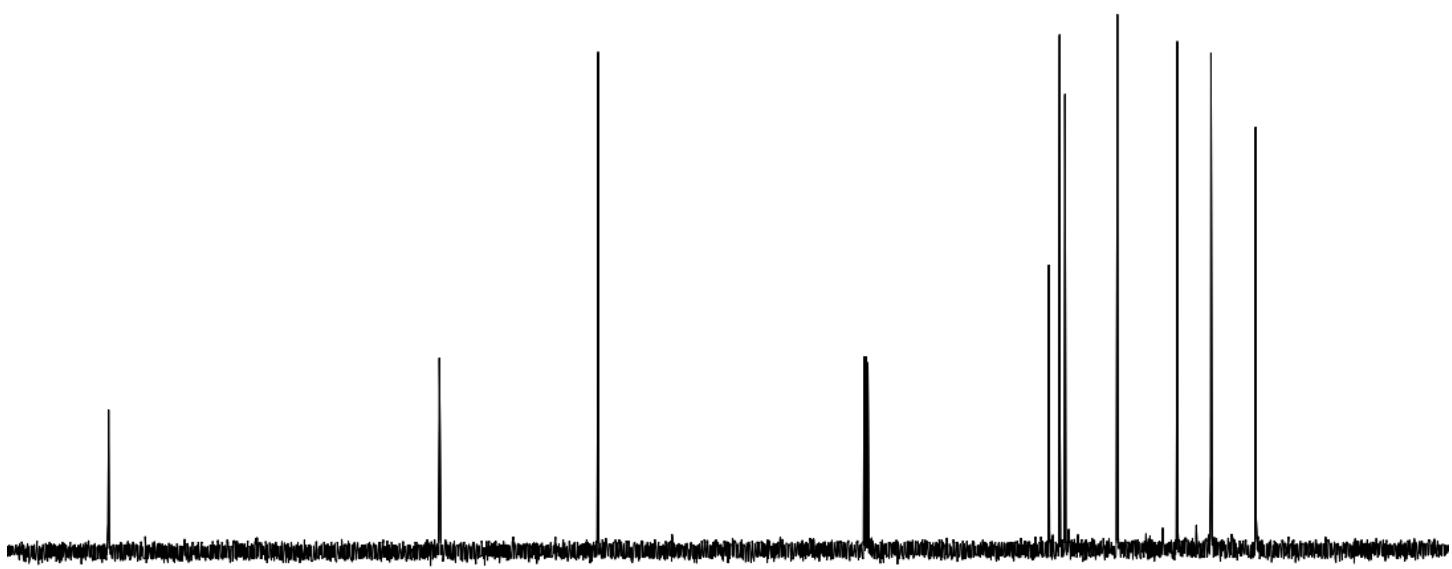

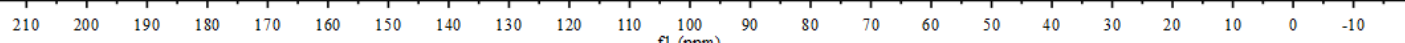


(c)

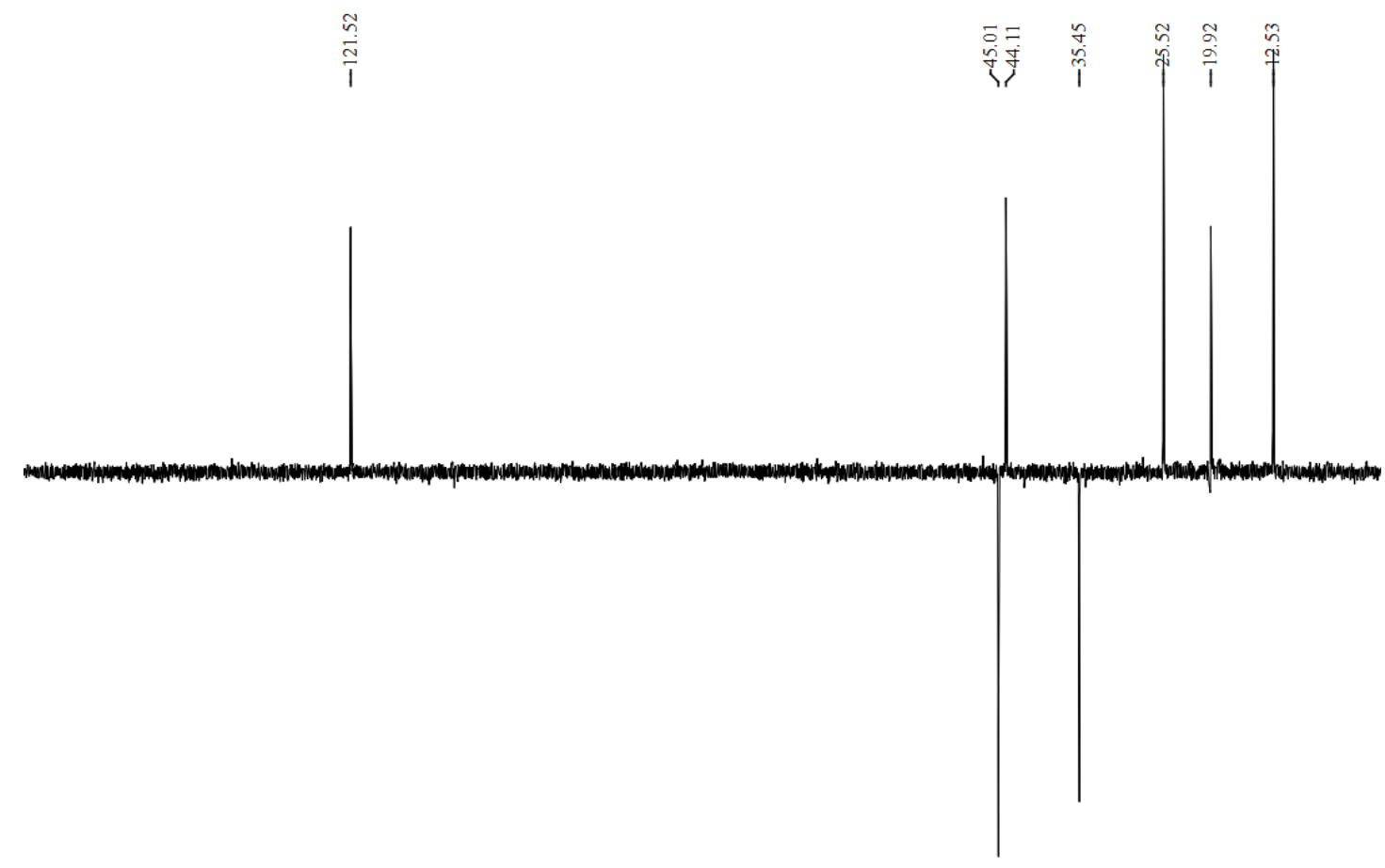

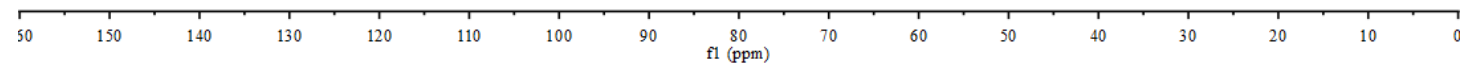

Figure S11. NMR spectra of compound Campholenaldehyde (2-(2,2,3-trimethylcyclopent-3-en-1-yl) -acetaldehyde): (a) ${ }^{1} \mathrm{H}$ NMR, (b) ${ }^{13} \mathrm{C}$ NMR, and (c) DEPT- 135. 
(a)

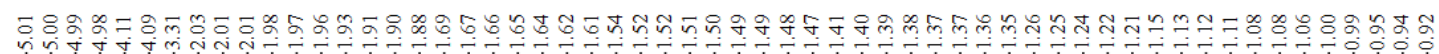
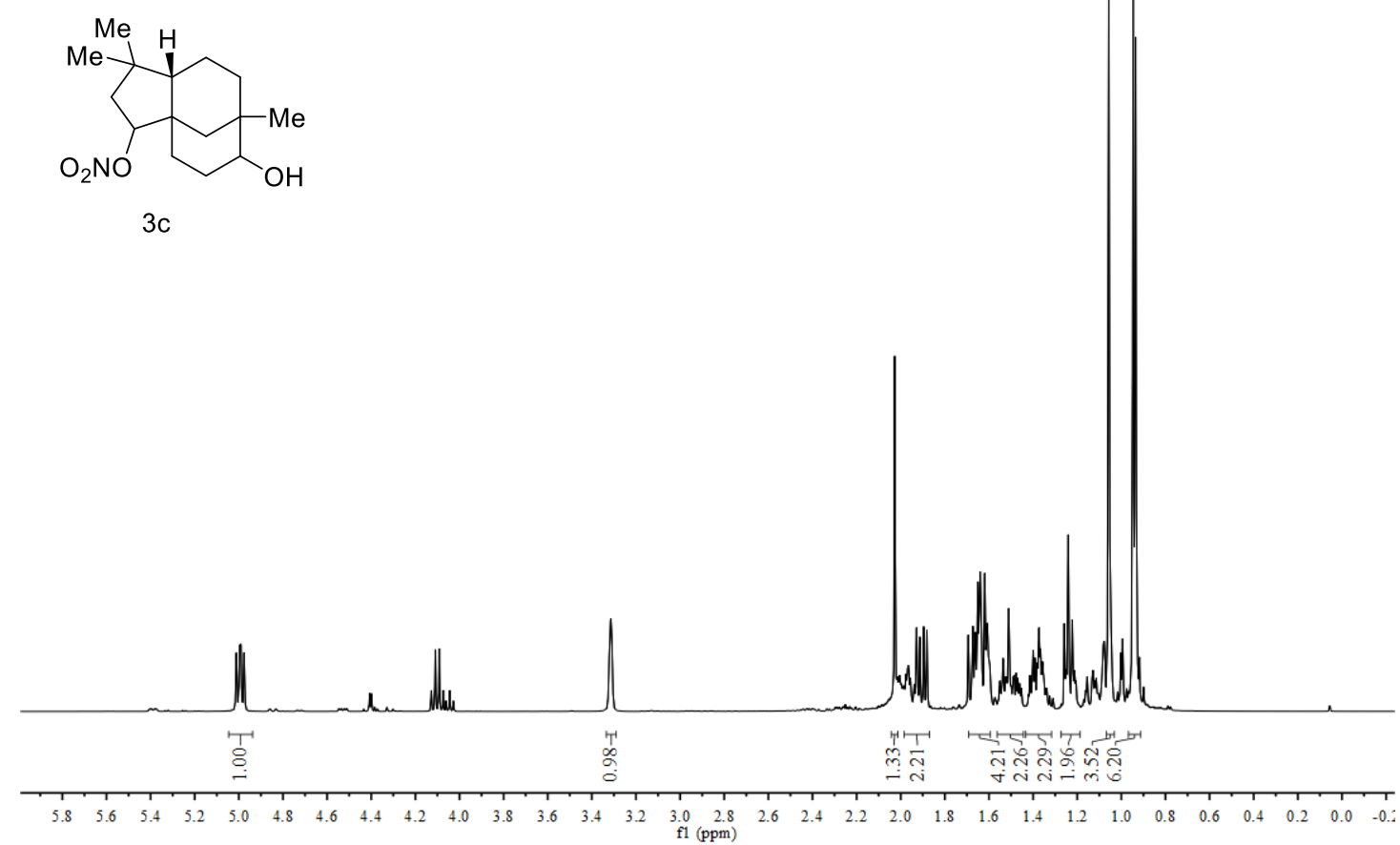

(b)

in
$i$
$i$

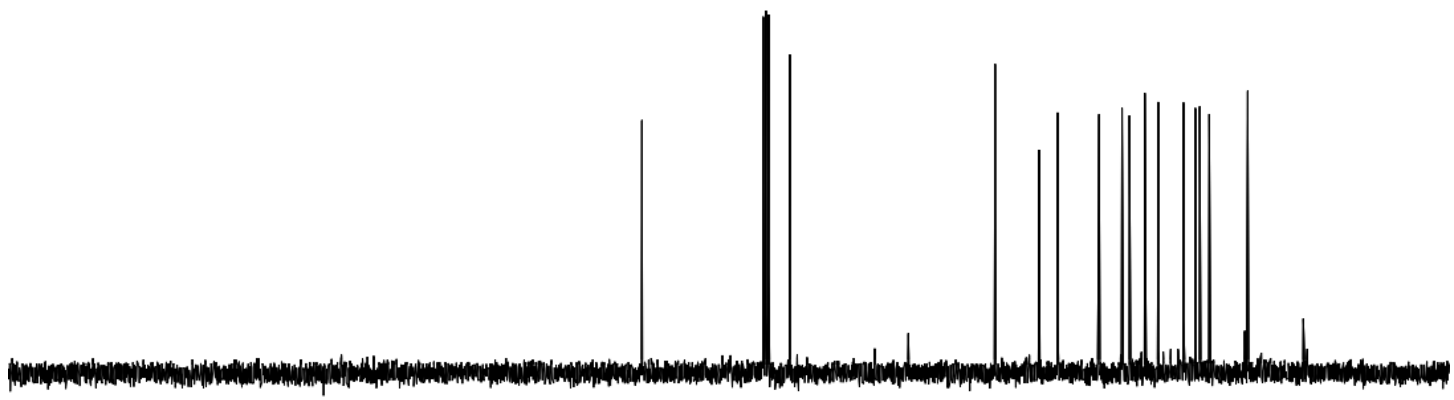

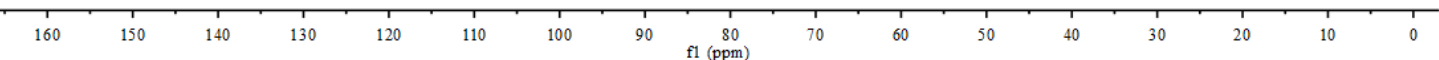


(c)
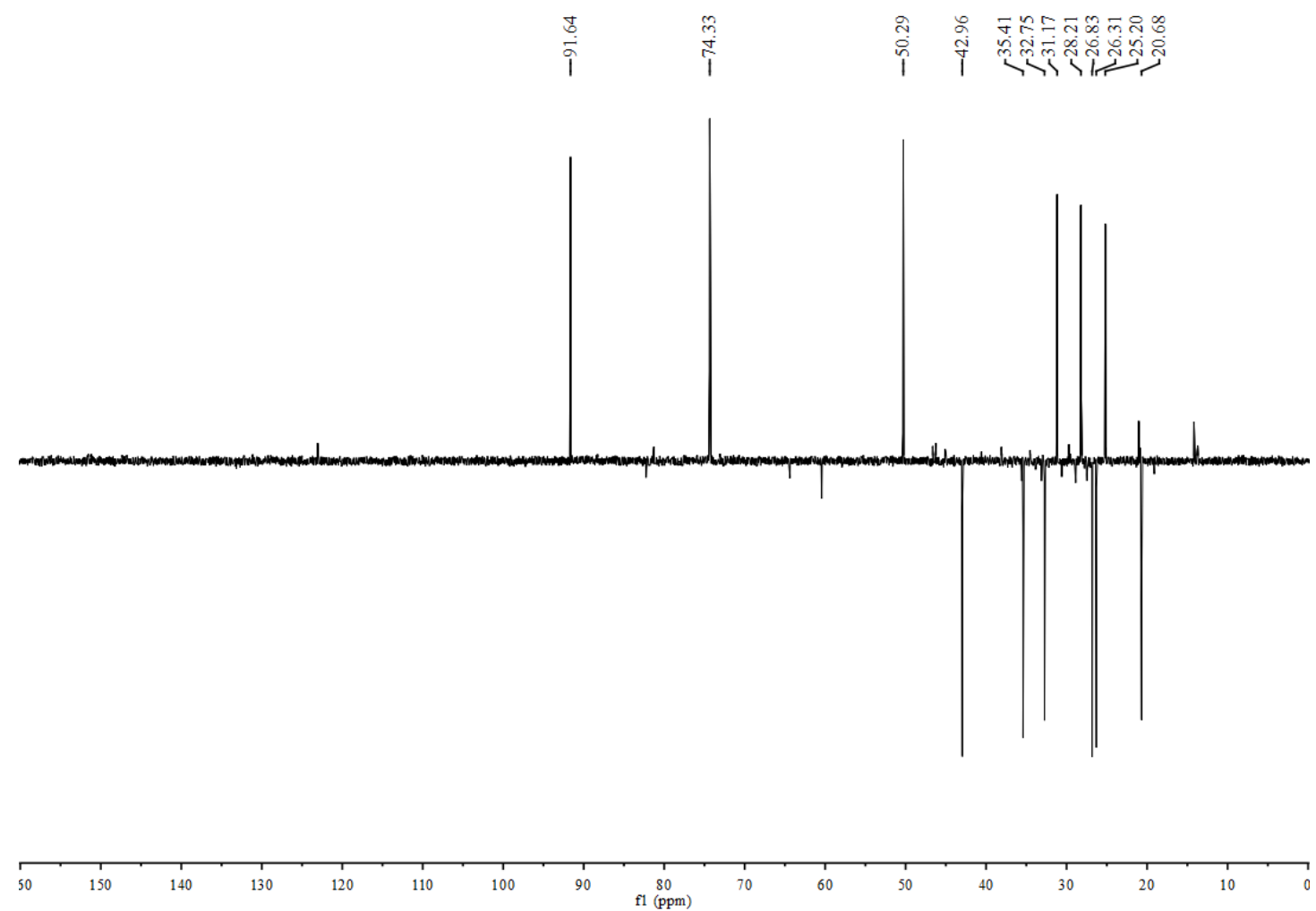

(d)

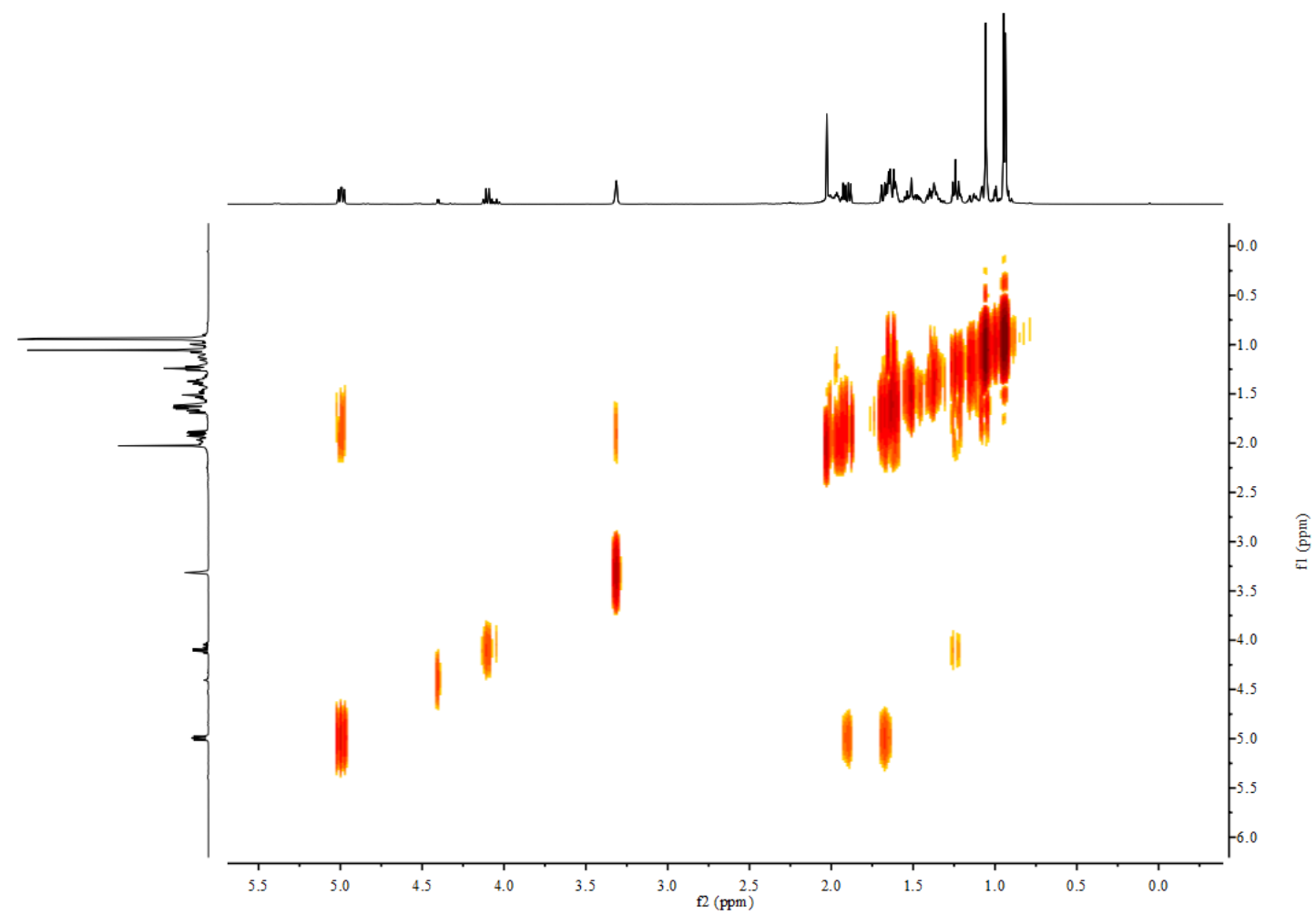


(e)

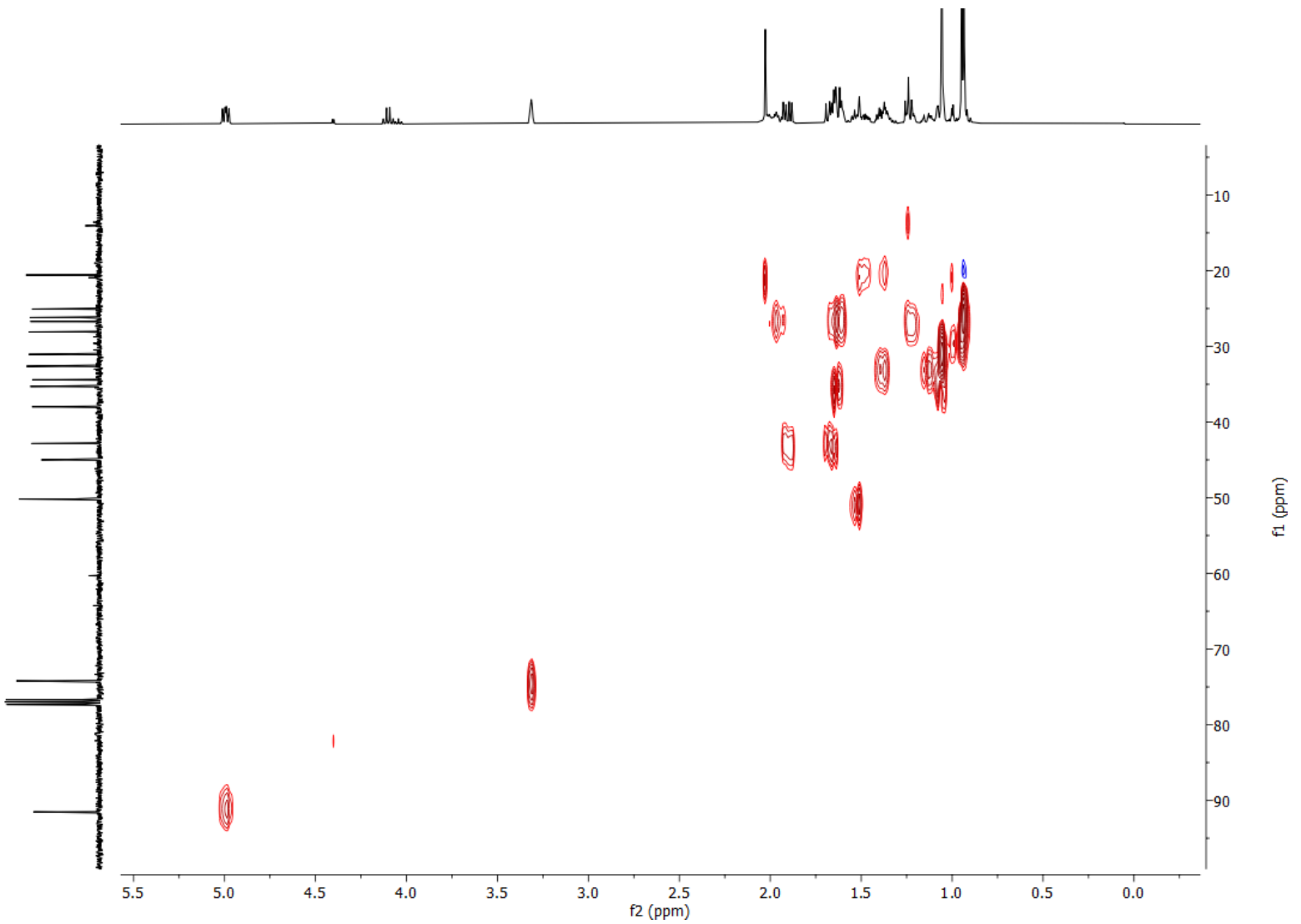

Figure S12. NMR spectra of compound 3c (6-hydroxy-1,1,7-trimethyldecahydro-3a,7 methanocyclopenta[8]annulen-3-yl nitrate): (a) ${ }^{1} \mathrm{H}$ NMR, (b) ${ }^{13} \mathrm{C}$ NMR, (c) DEPT-135, (d) COSY, and (e) HSQC. 
(a)
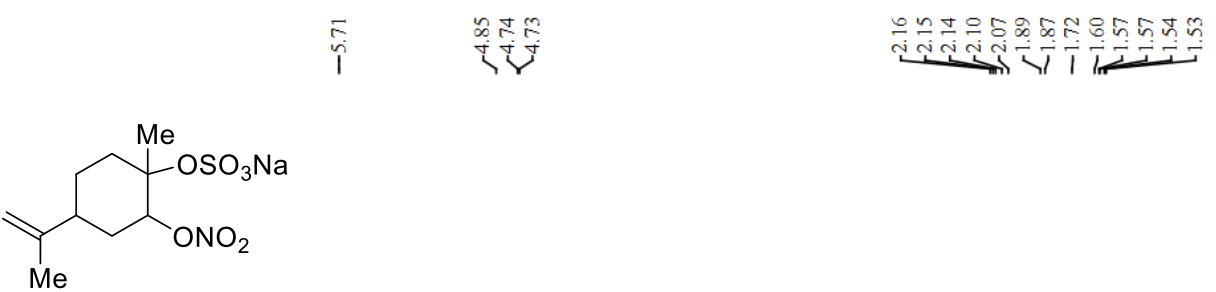

$4 b^{\prime}=2^{\circ}$ LmNOS

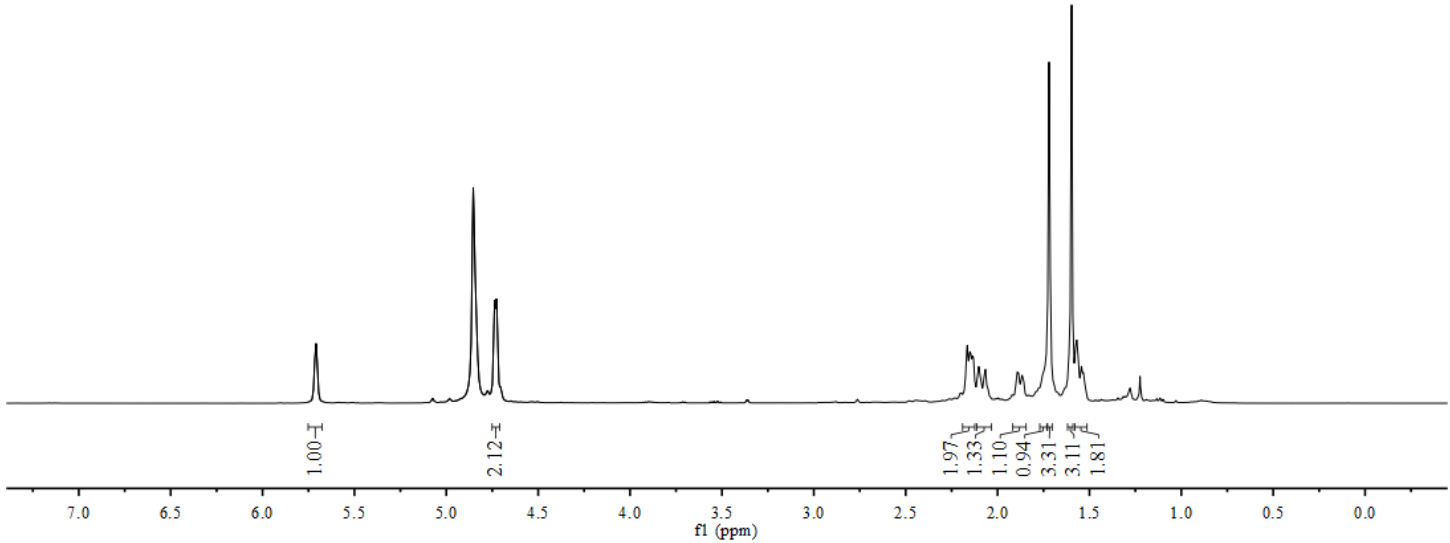

(b)

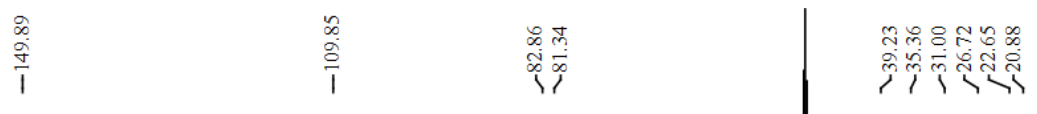


(c)
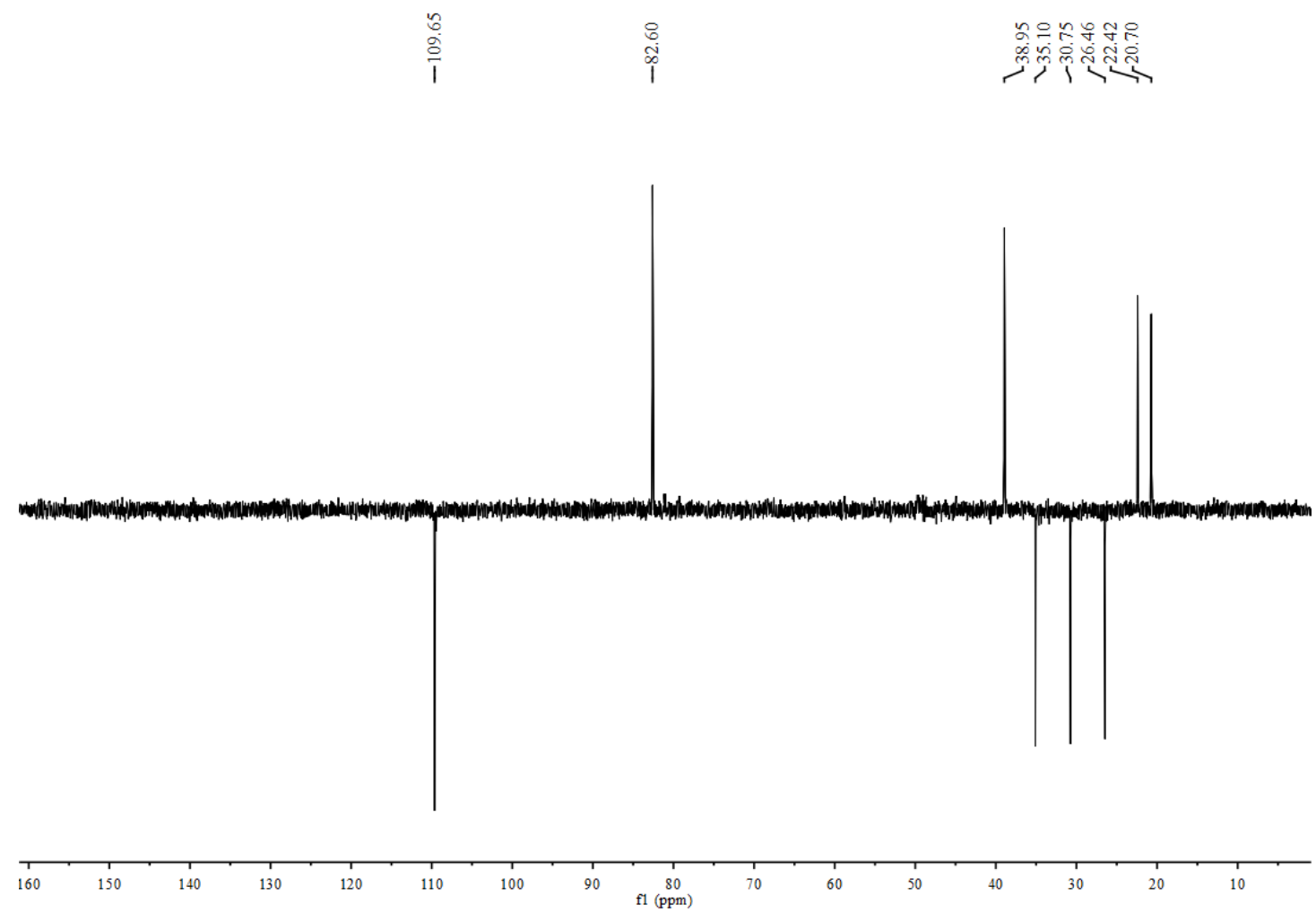

(d)

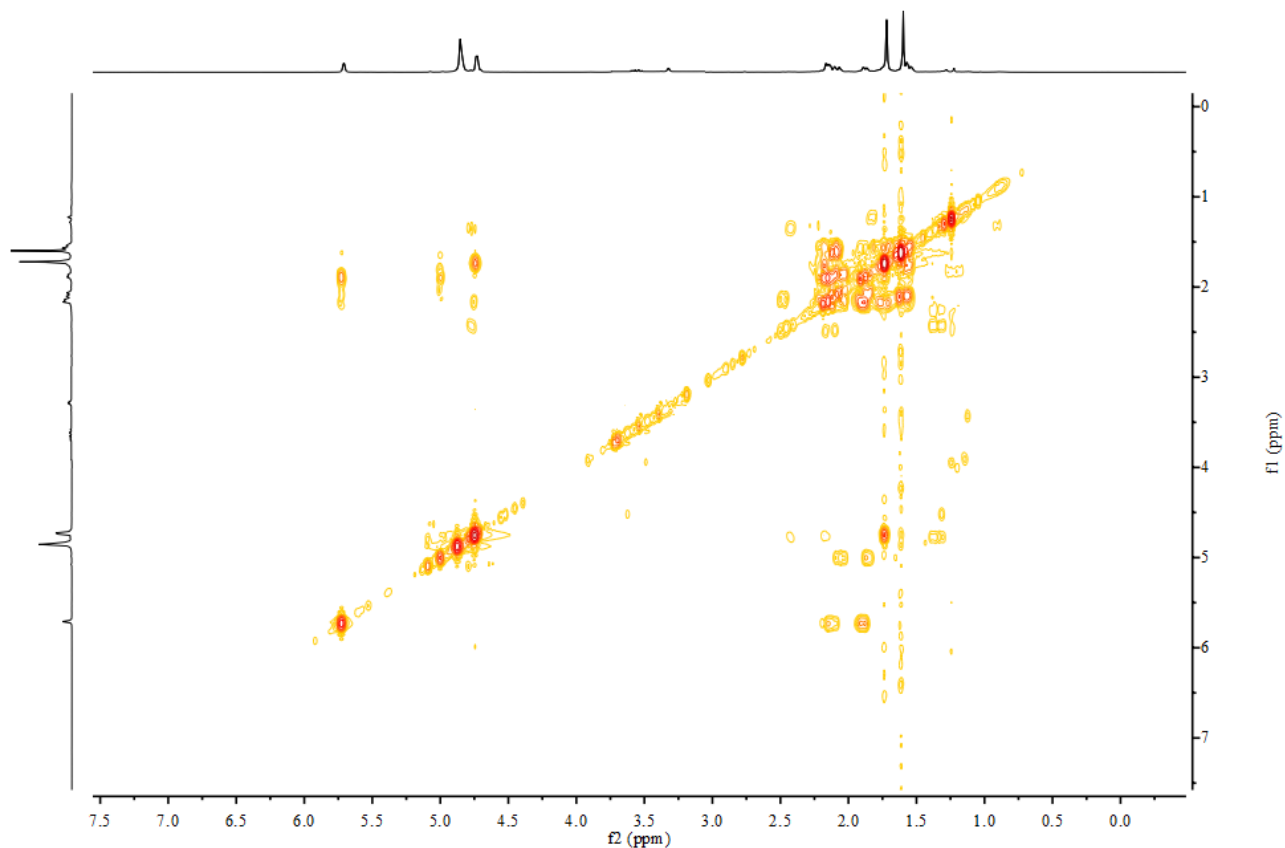


(e)

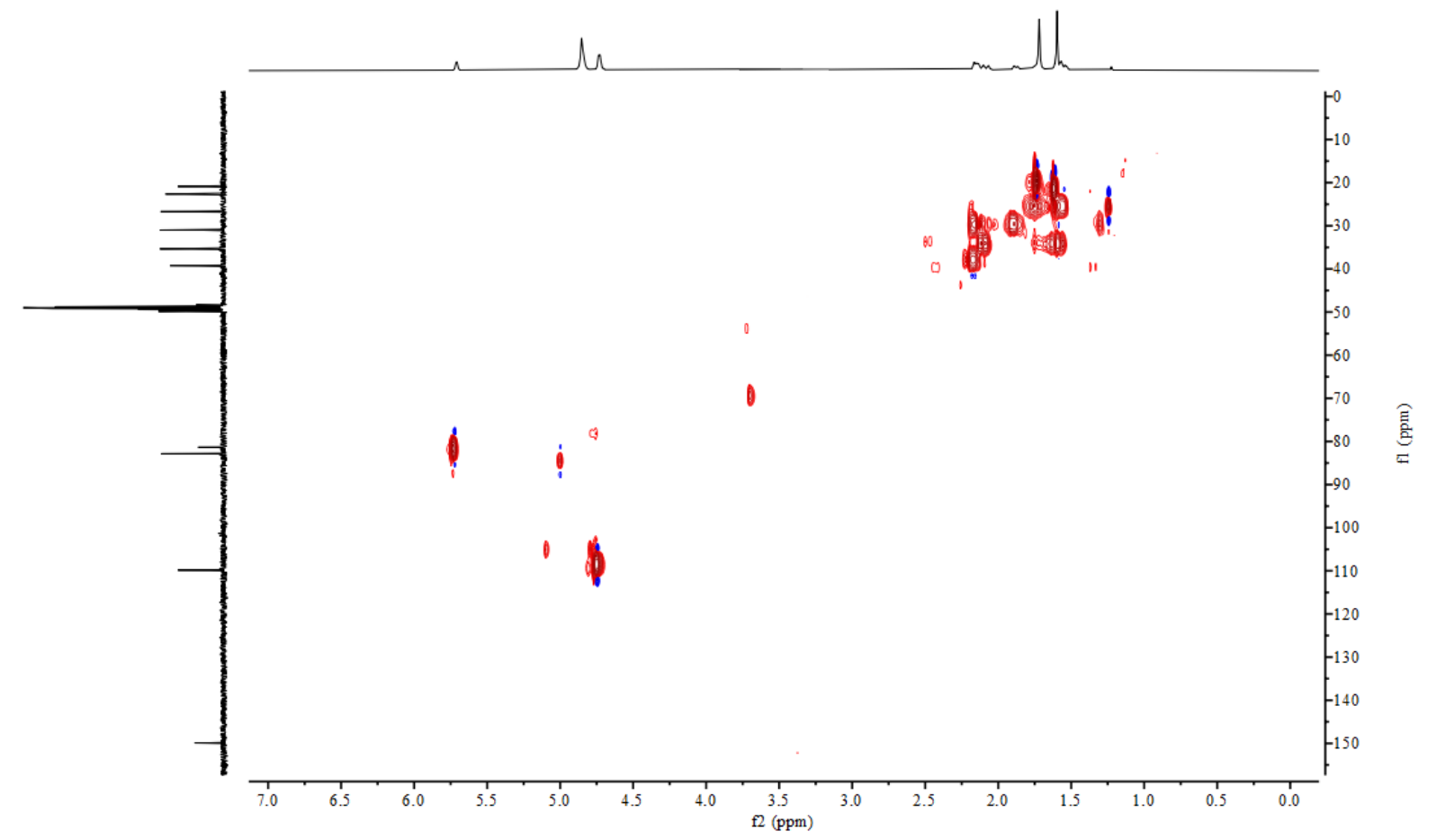

Figure S13. NMR spectra of compound 4b' (sodium-1-methyl-2-(nitrooxy)-4

-(prop-1-en-2-yl) cyclohexyl sulfate): (a) ${ }^{1} \mathrm{H}$ NMR, (b) ${ }^{13} \mathrm{C}$ NMR. (c) DEPT-135, (d) COSY, and (e) HSQC. 
(a)

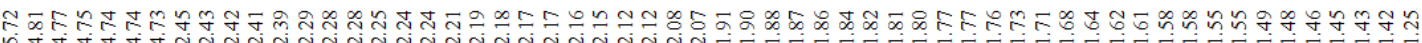

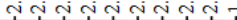

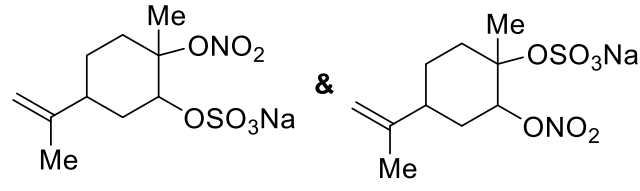

$4 \mathrm{~b}=3^{\circ}{ }_{-}$LmNOS $\quad 4 b^{\prime}=2^{\circ}{ }_{-}$LmNOS

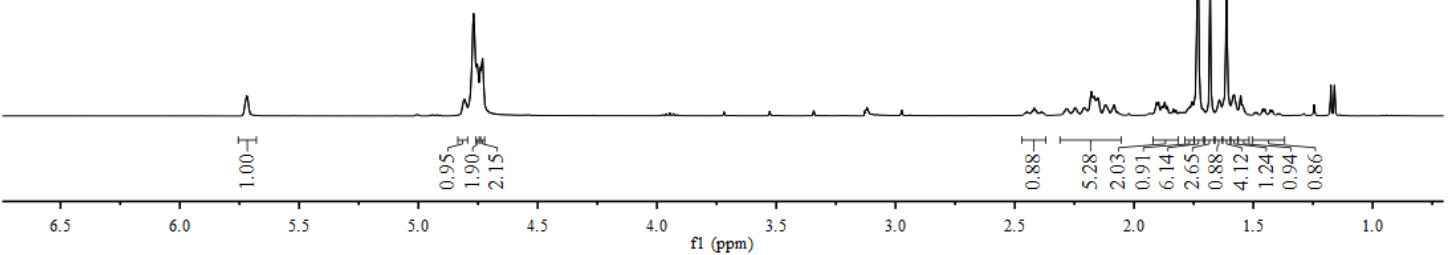

(b)

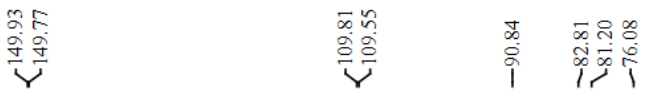

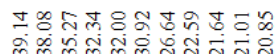

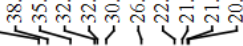
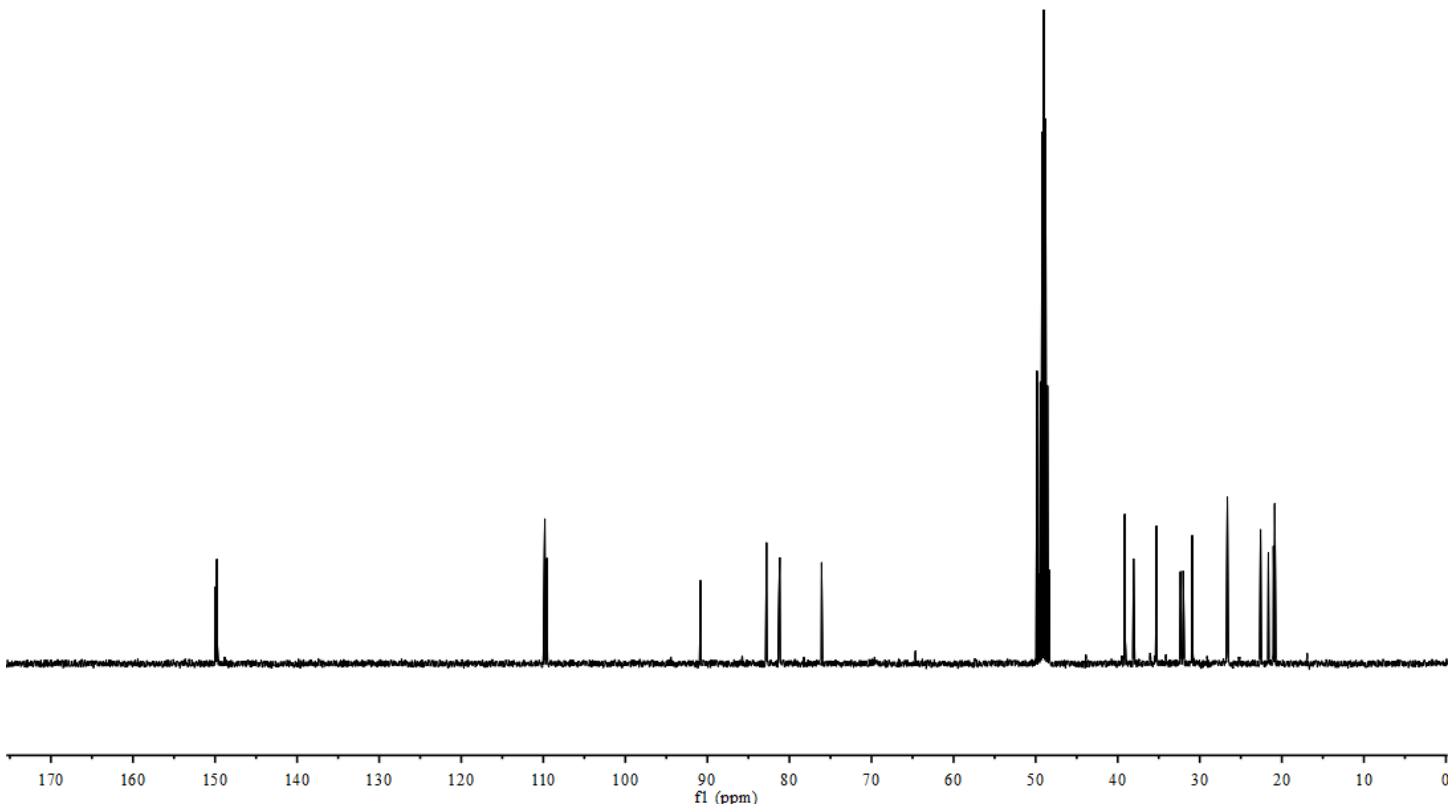
(c)

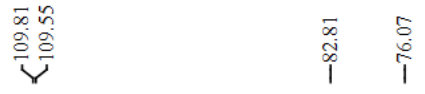

무에

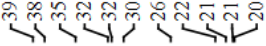
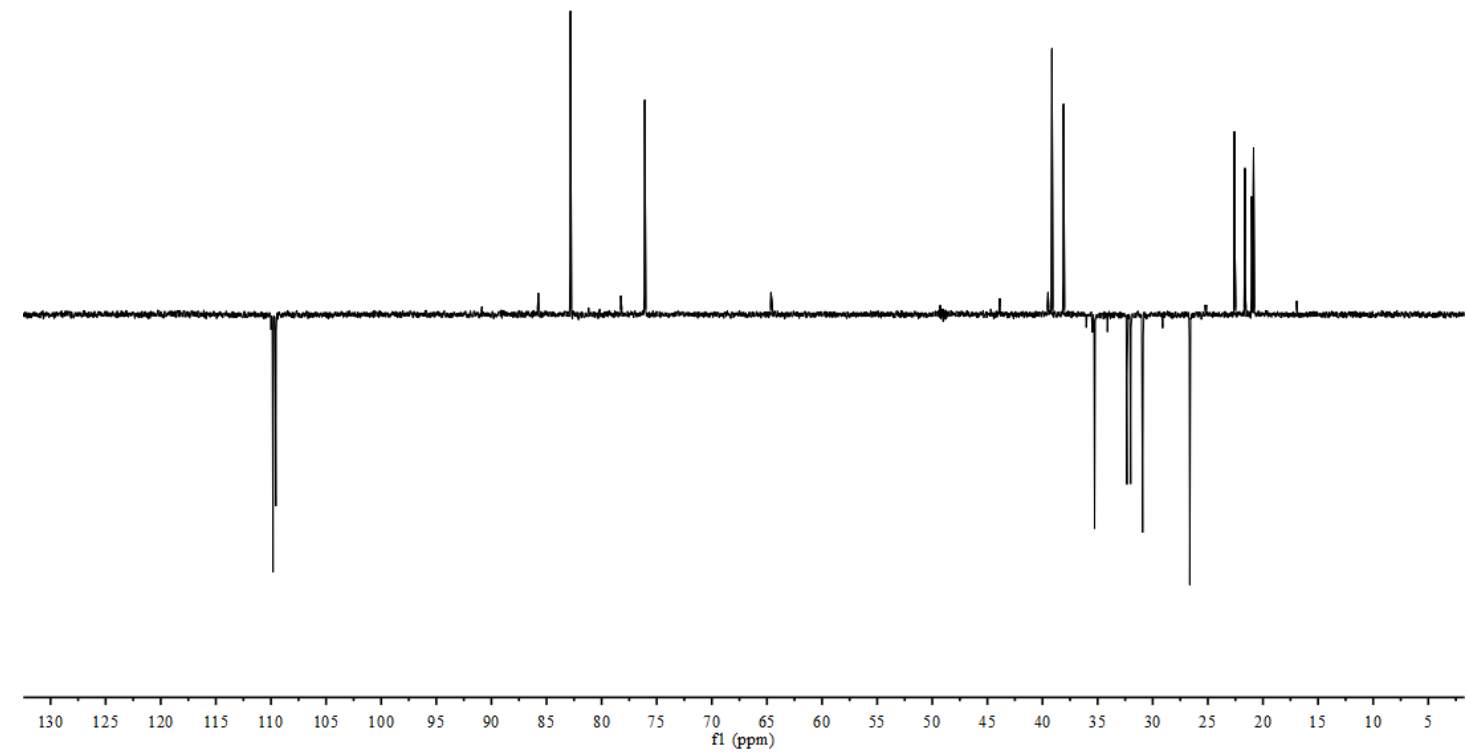

(d)

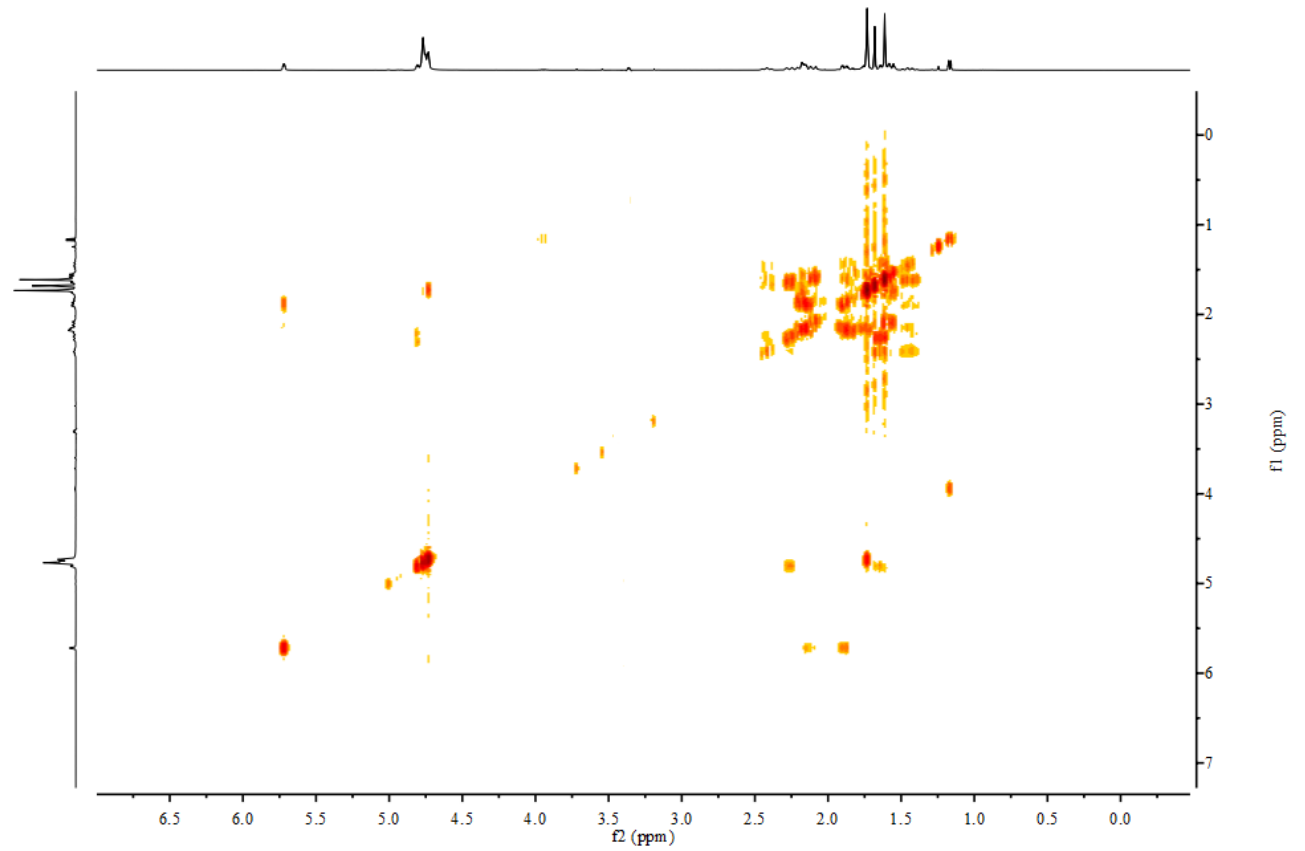


(e)

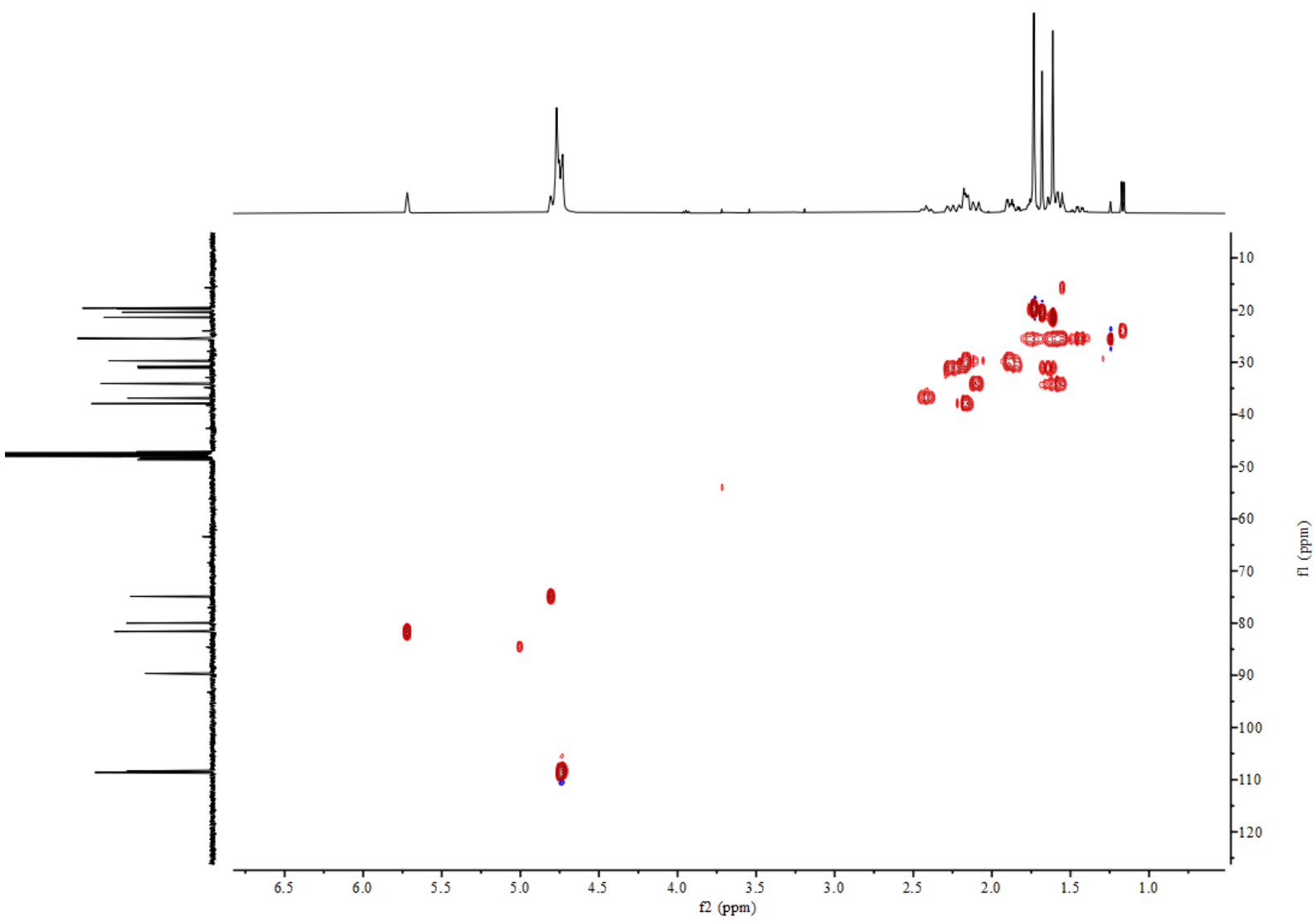

Figure S14. NMR spectra of compound 4b' (sodium 1-methyl-2-(nitrooxy)-4-(prop-1-en-2yl)cyclohexyl sulfate) \& $\mathbf{4 b}$ (sodium 2-methyl-2-(nitrooxy)-5-(prop-1-en-2-yl)cyclohexyl sulfate). (a) ${ }^{1} \mathrm{H}$ NMR, (b) ${ }^{13} \mathrm{C}$ NMR, (c) DEPT-135, (d) COSY, and (e) HSQC. 
(a)

乡in

نinin

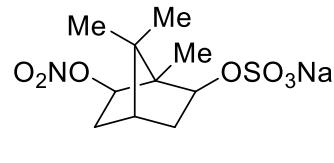

$4 a^{\prime}=2^{\circ} \_$ApNOS

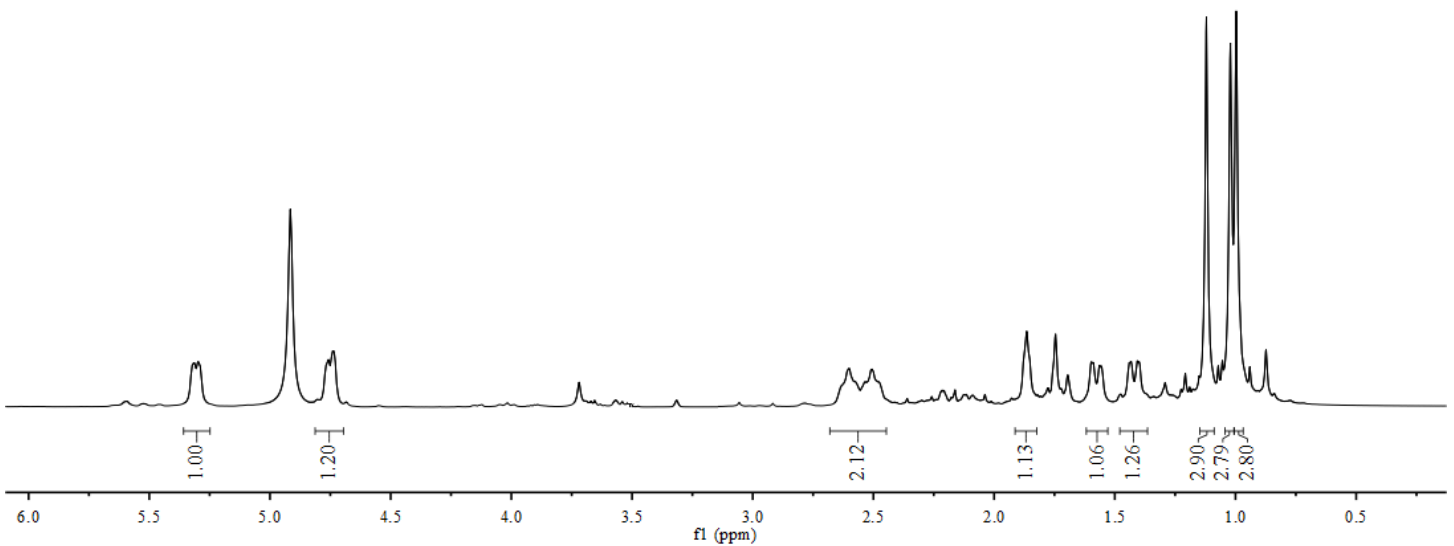

(b)

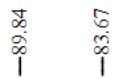


(c)

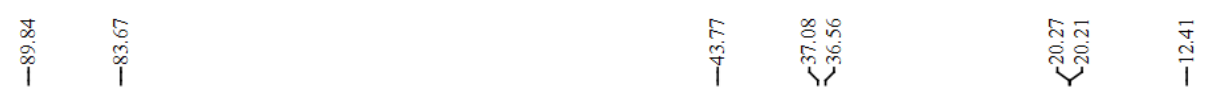
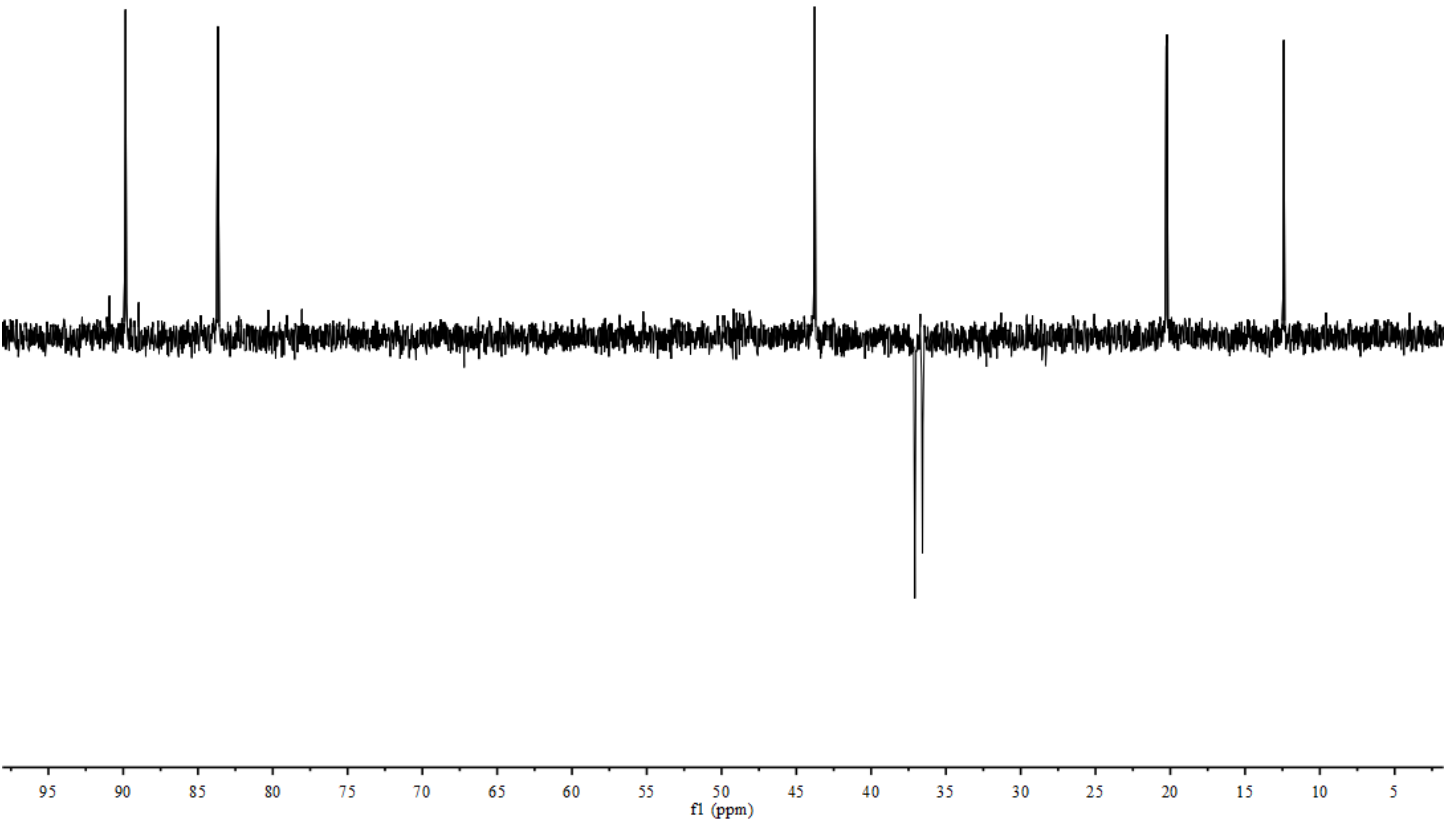

(d)

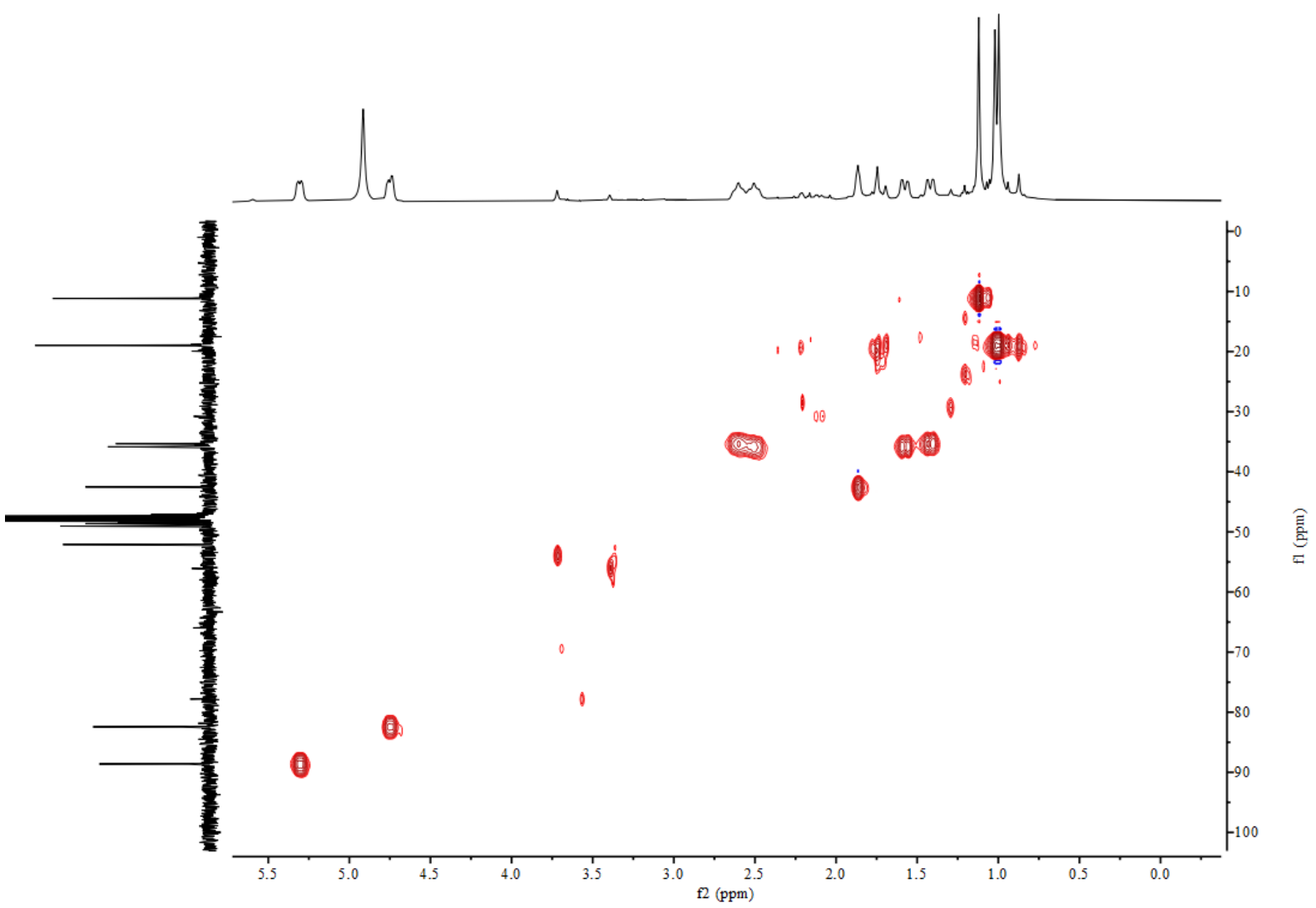

Figure S15. NMR spectra of compound 4a' (sodium 1,7,7-trimethyl-6-(nitrooxy) bicyclo[2.2.1] heptan-2-yl sulfate): (a) ${ }^{1} \mathrm{H}$ NMR, (b) ${ }^{13} \mathrm{C}$ NMR, (c) DEPT-135, and (d) HSQC. 
(a)

$\stackrel{8}{i}$
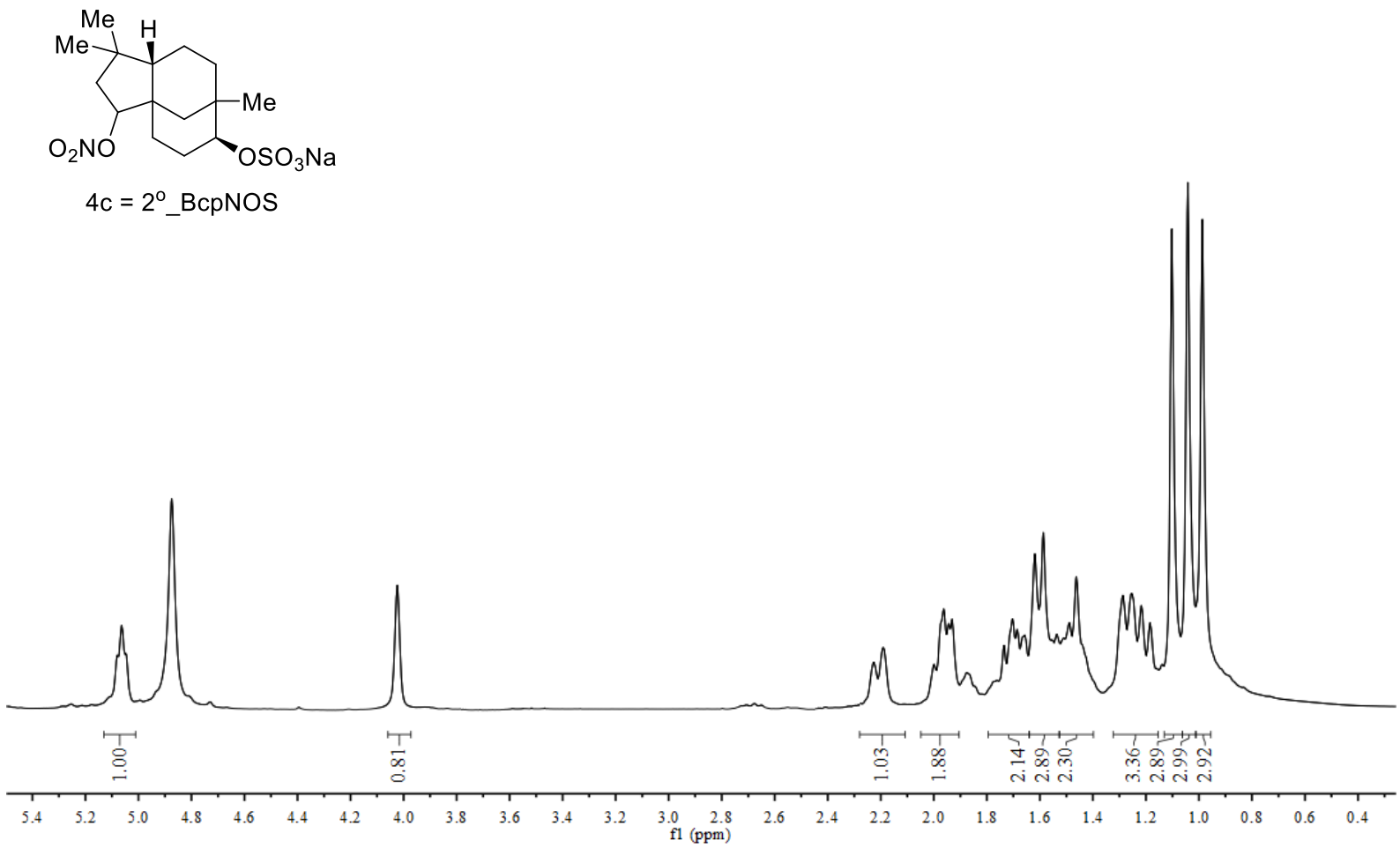

(b)

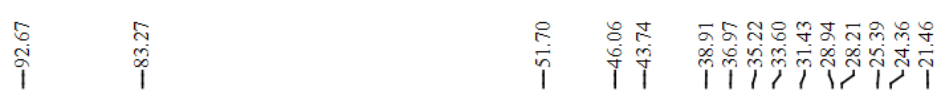

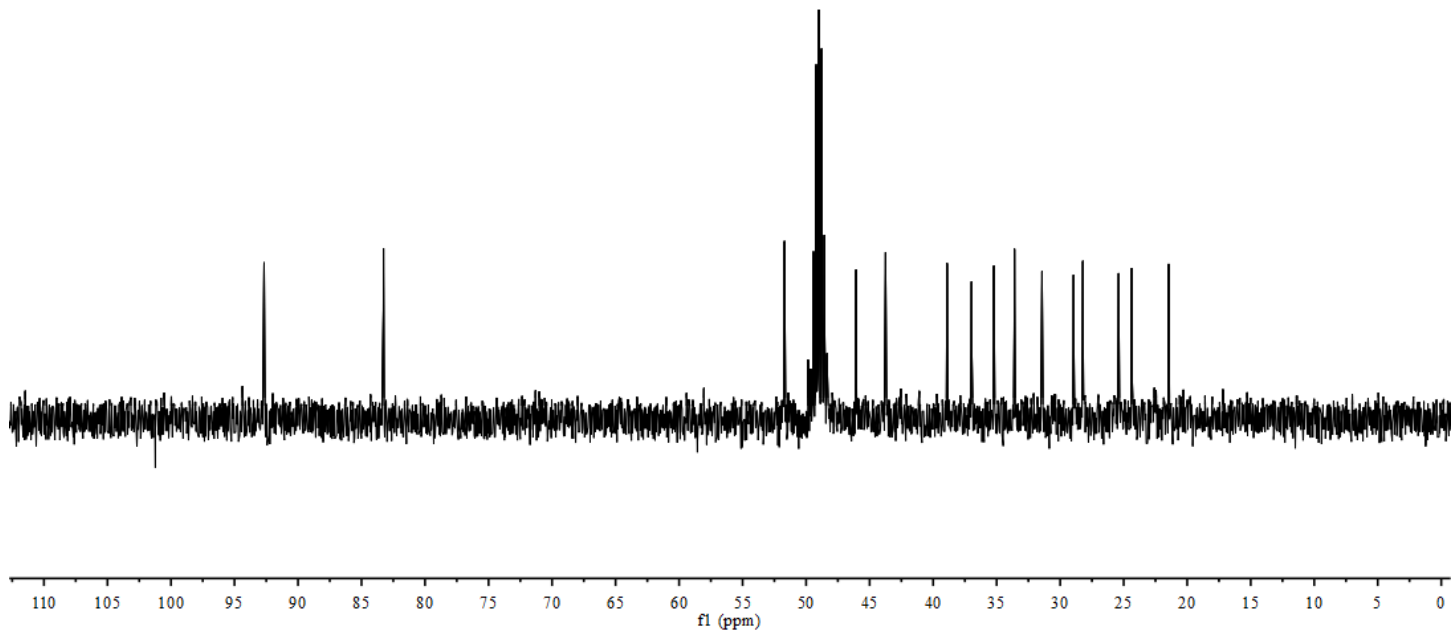


(c)

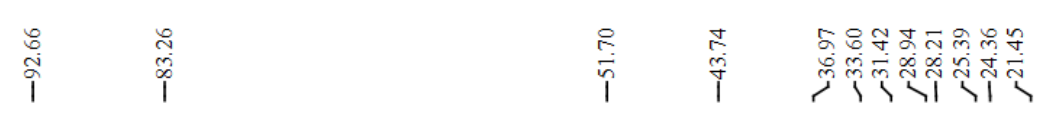

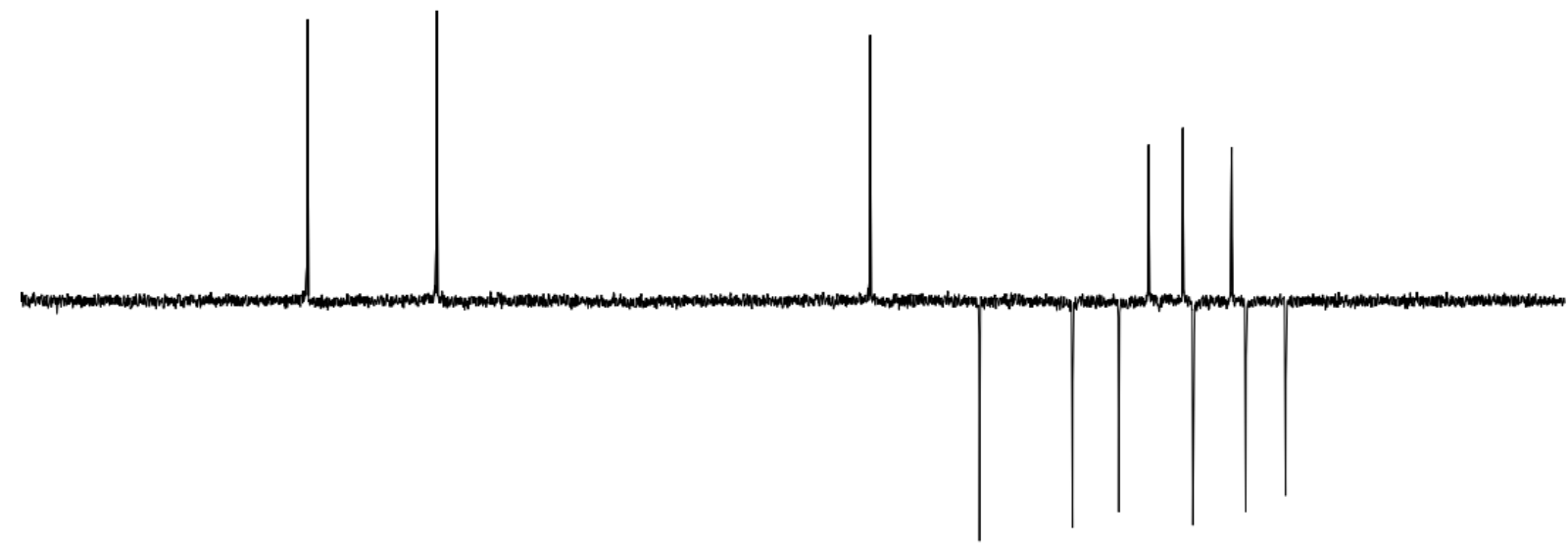

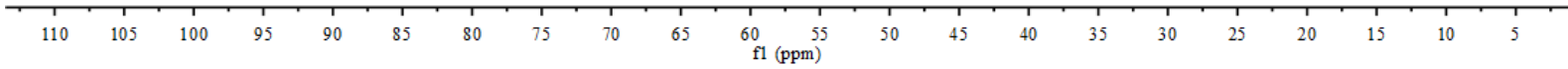

(d)

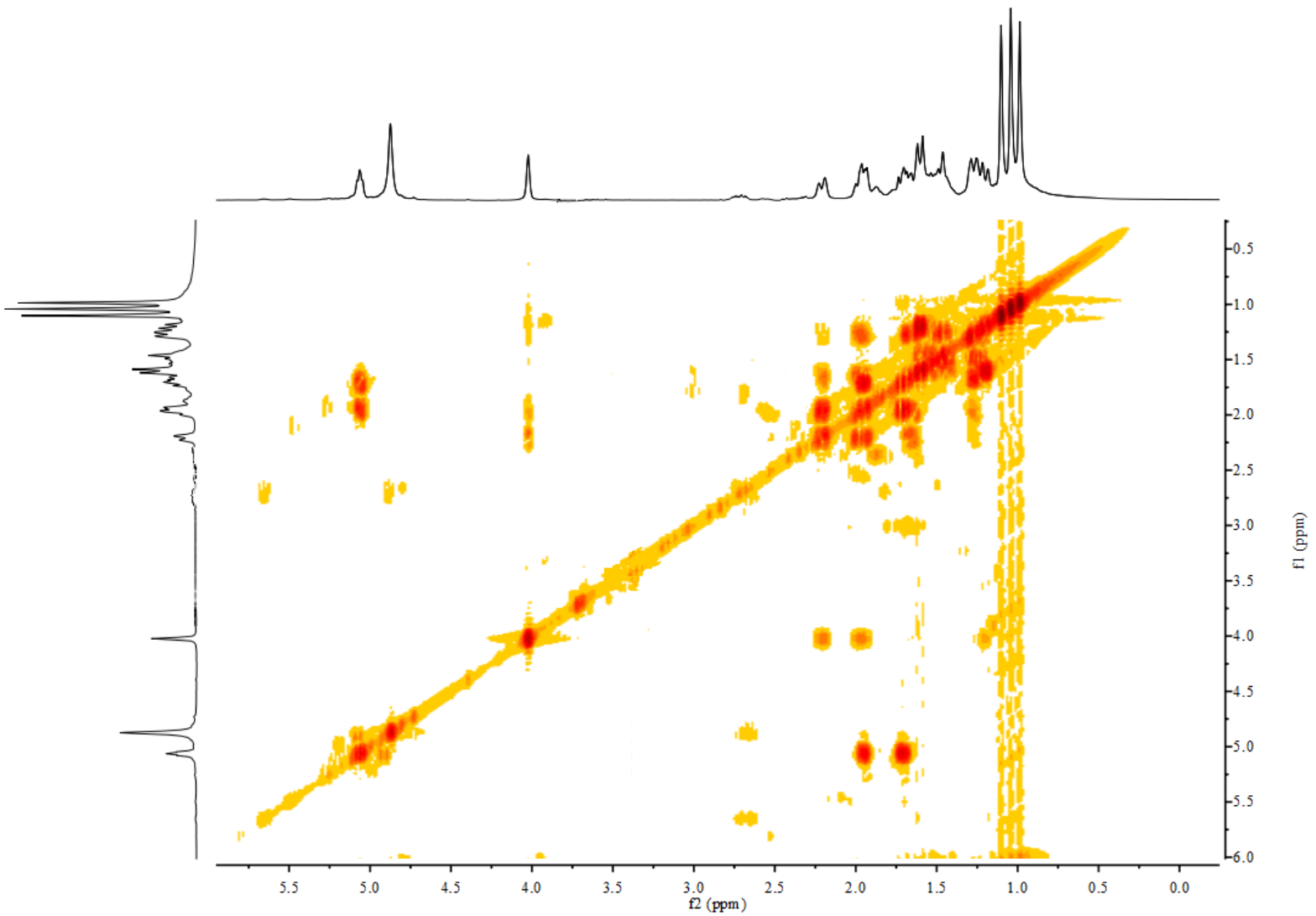


(e)

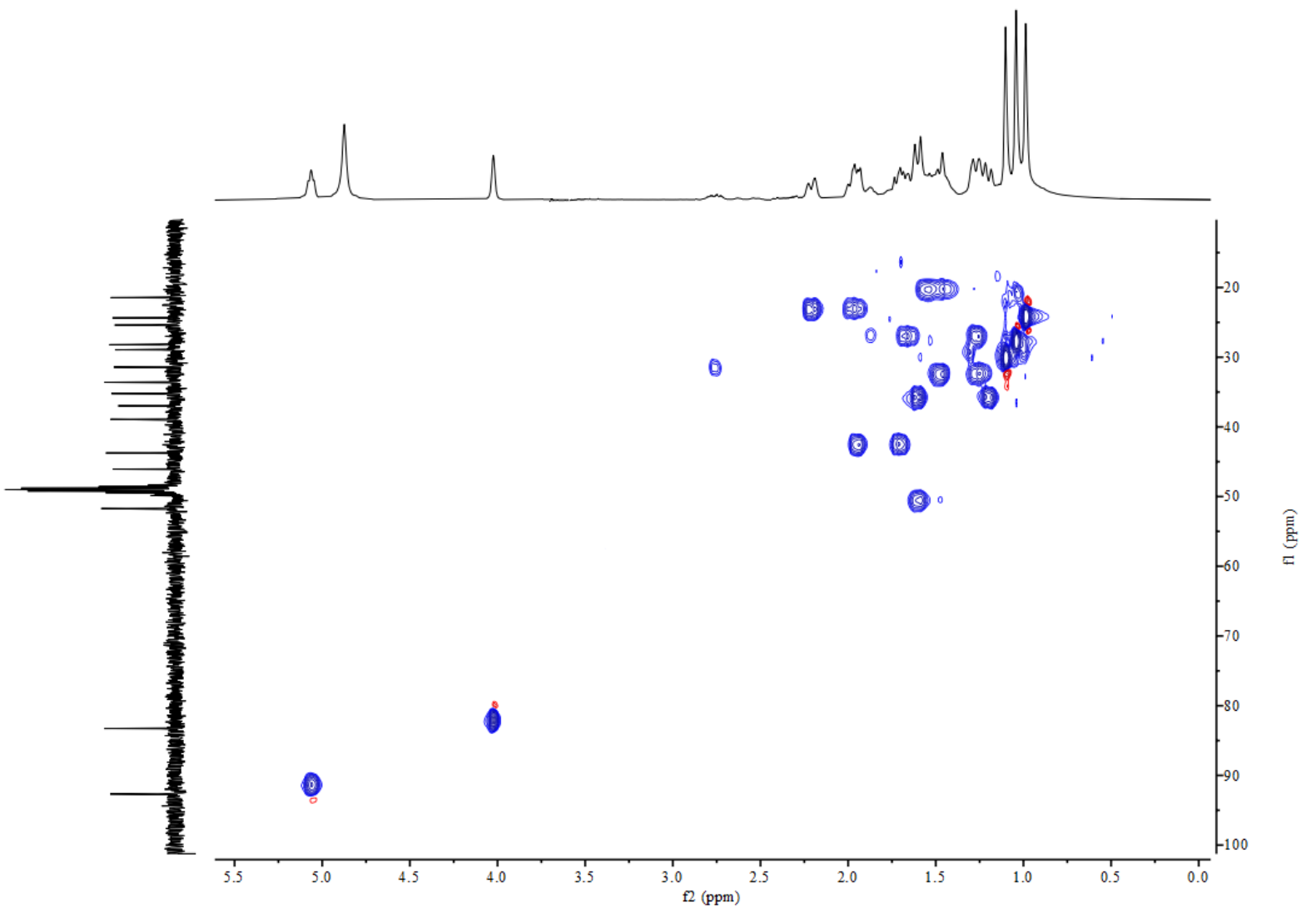

Figure S16. NMR spectra of compound 4c (sodium 1,1,7-trimethyl-3-(nitrooxy) -decahydro-3a,7methanocyclopenta-[8]annulen-6-yl sulfate) (a) ${ }^{1} \mathrm{H}$ NMR, (b) ${ }^{13} \mathrm{C}$ NMR, (c) DEPT-135, (d) COSY, and (e) HSQC. 
(a)

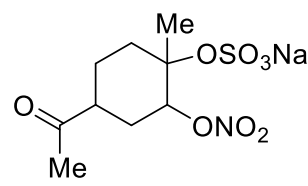

$5 b^{\prime}=2^{\circ}{ }_{-}$LkNOS

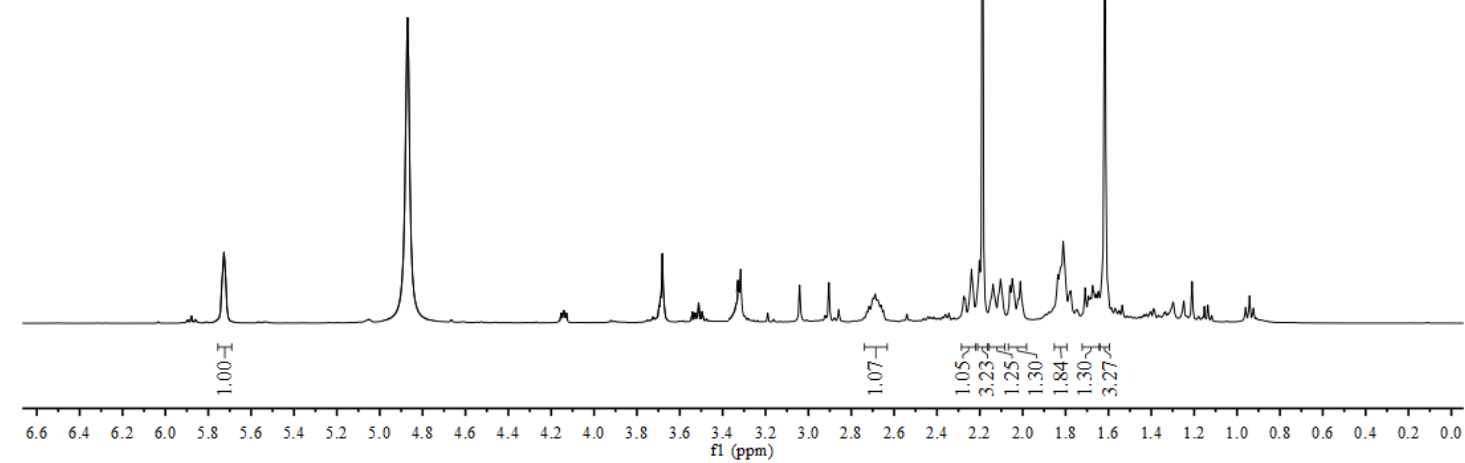

(b)
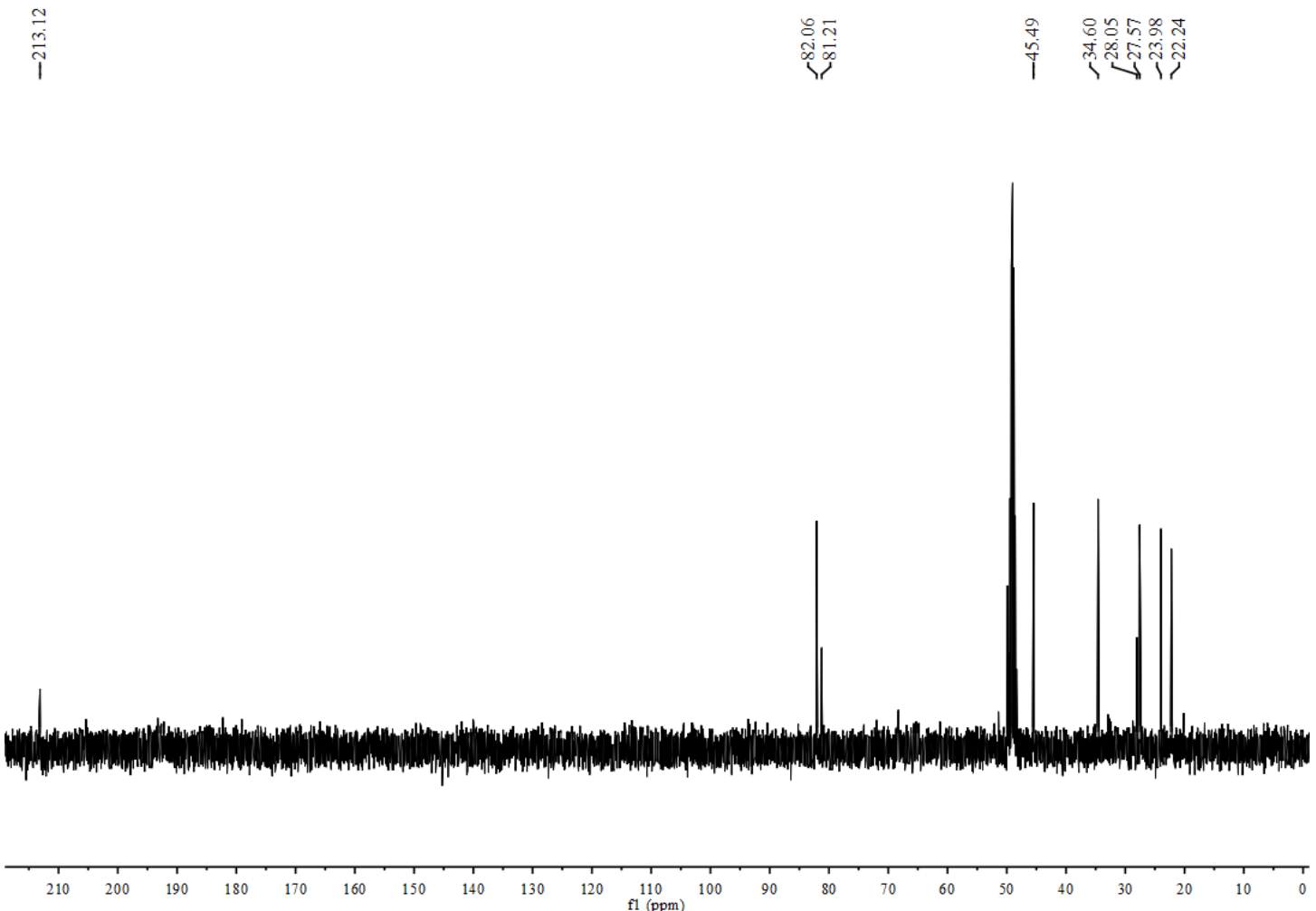
(c)
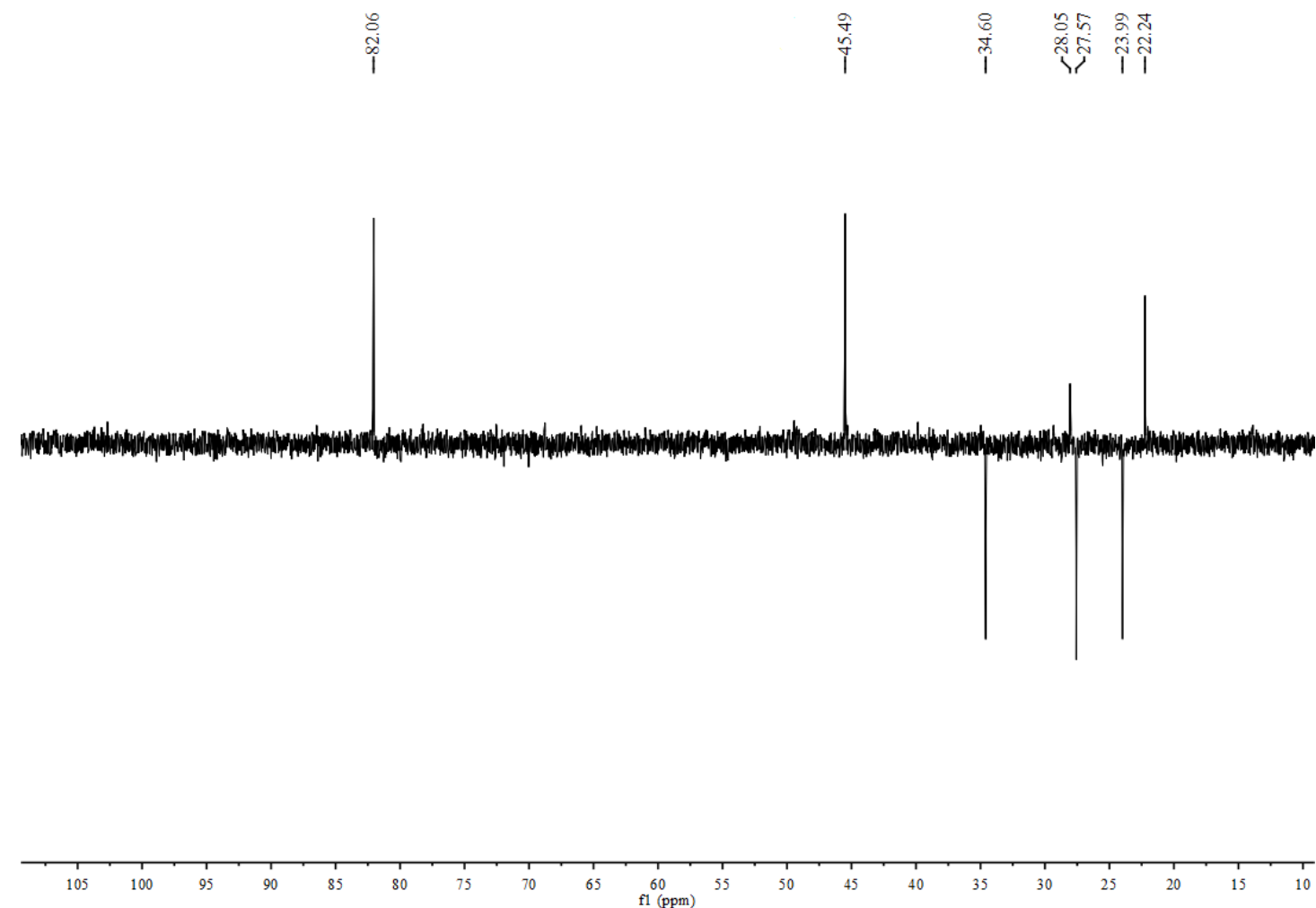

(b)

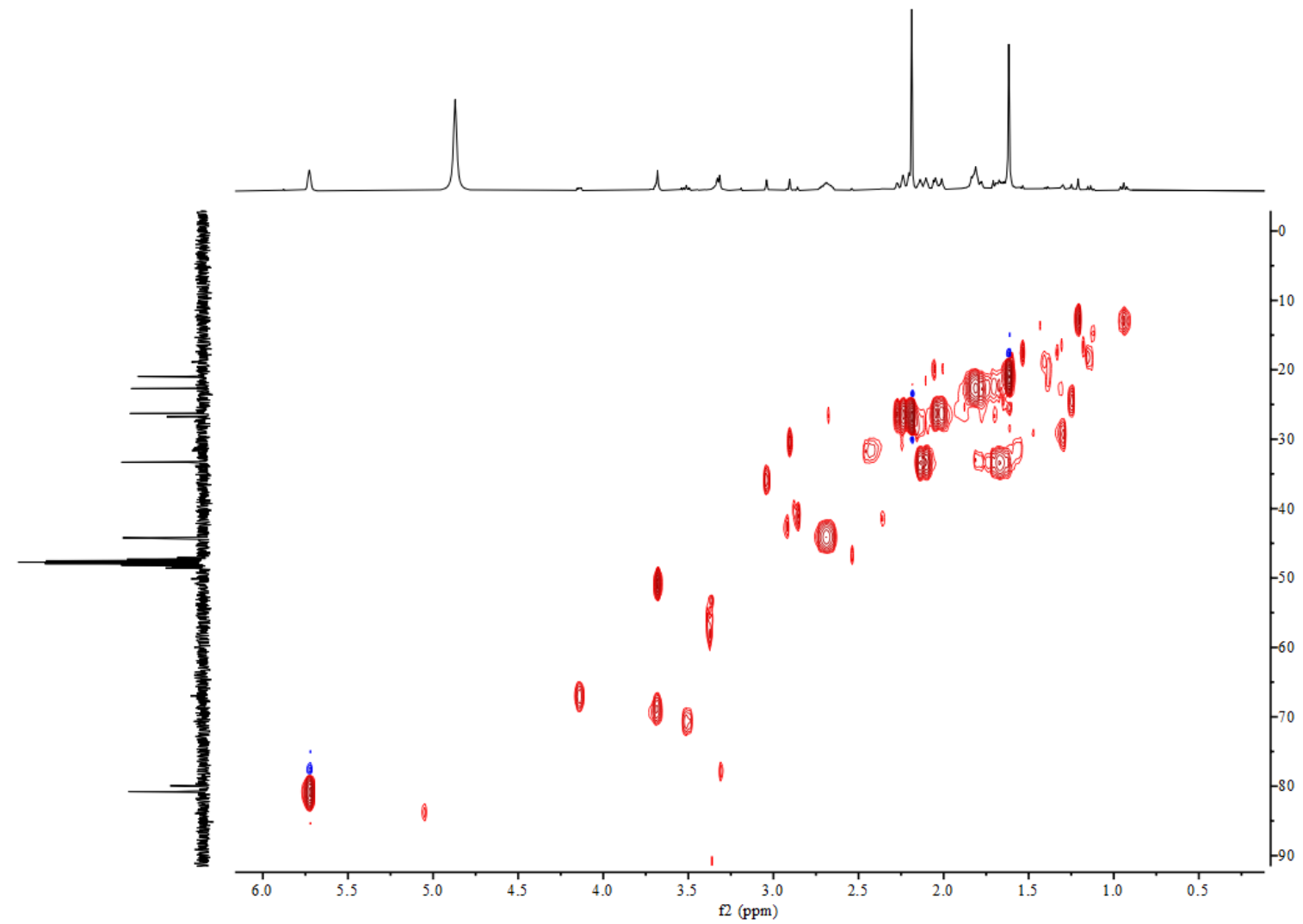

Figure S17. NMR spectra of compound 5b' (sodium 4-acetyl-1-methyl-2-(nitrooxy) -cyclohexyl sulfate): (a) ${ }^{1} \mathrm{H}$ NMR, (b) ${ }^{13} \mathrm{C}$ NMR, (c) DEPT-135, and (d) HSQC. 
S4. Hydrolysis of 3a in NMR

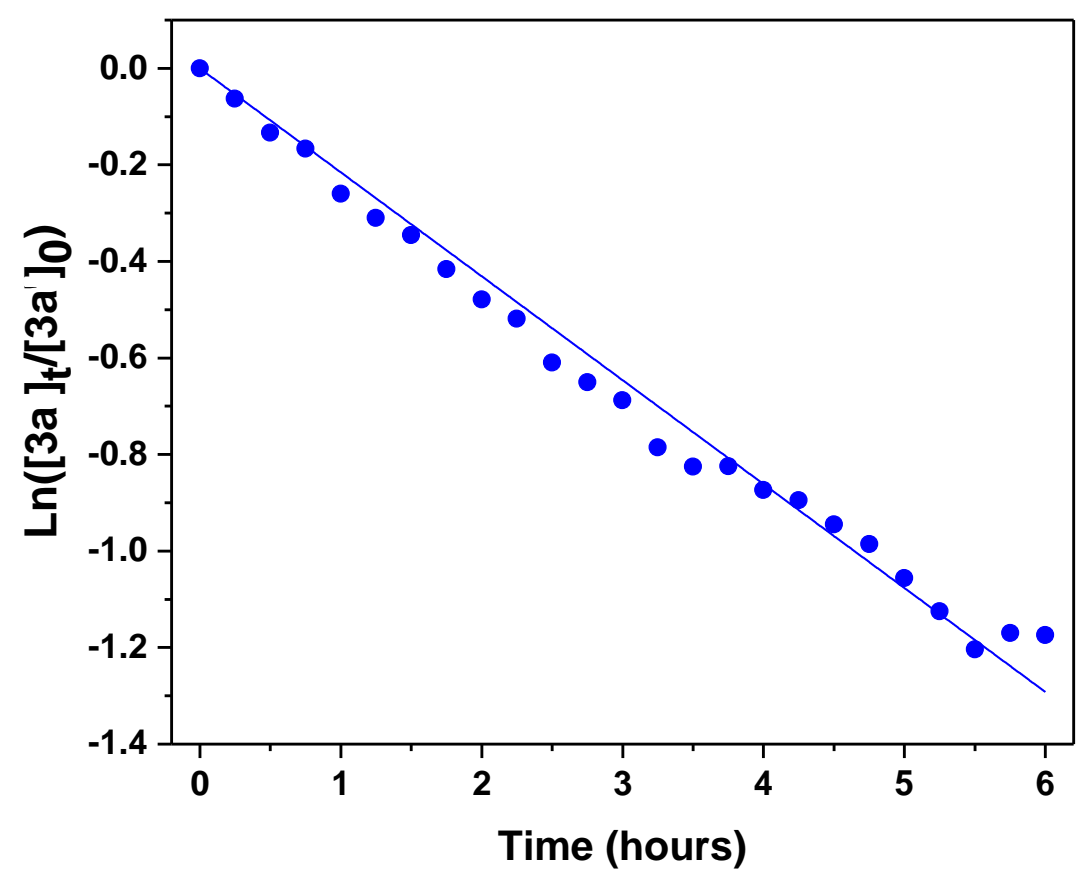

Figure S18. Pseudo first order kinetic analysis for hydrolysis of $\mathbf{3 a}$ in $\mathrm{CD}_{3} \mathrm{OD}$ detected by in situ NMR. 
S5. MS/MS data collected from ambient samples compared to authentic standards

(a)

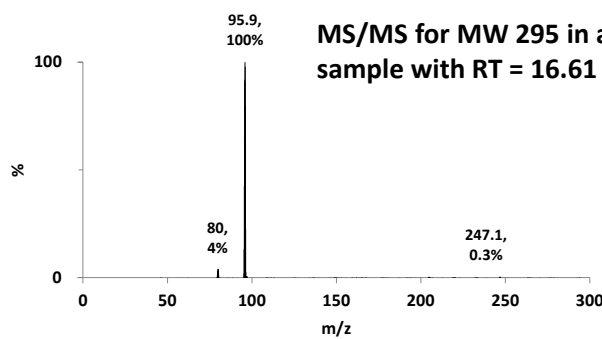

(c) 100

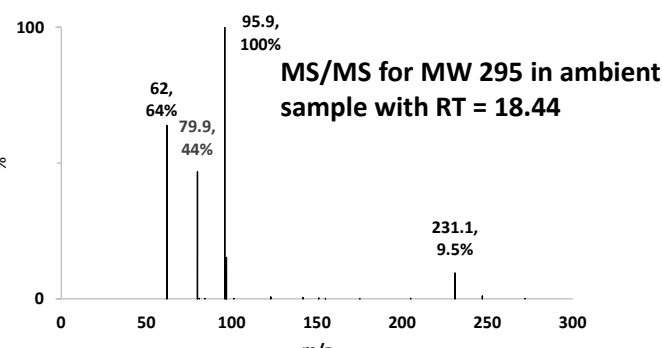

(e)

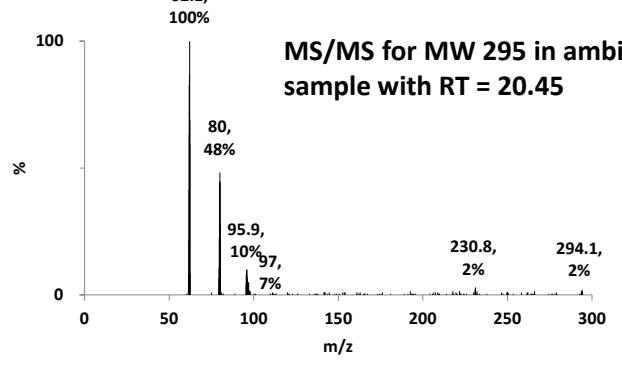

(g)

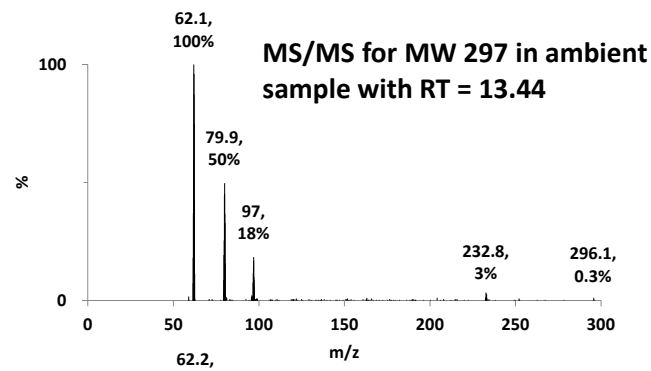

(i)
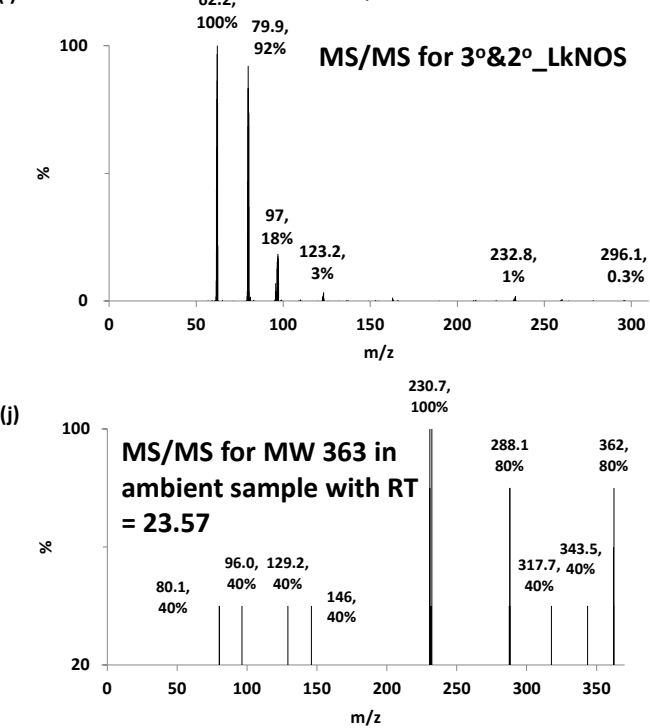
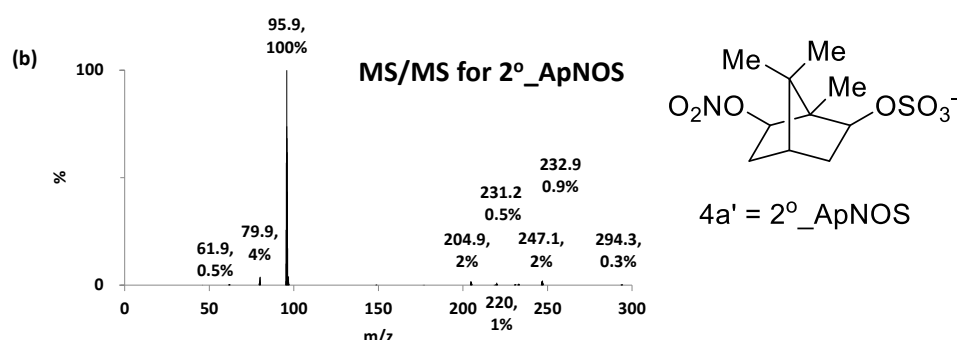

$4 a^{\prime}=2^{\circ} \_$ApNOS

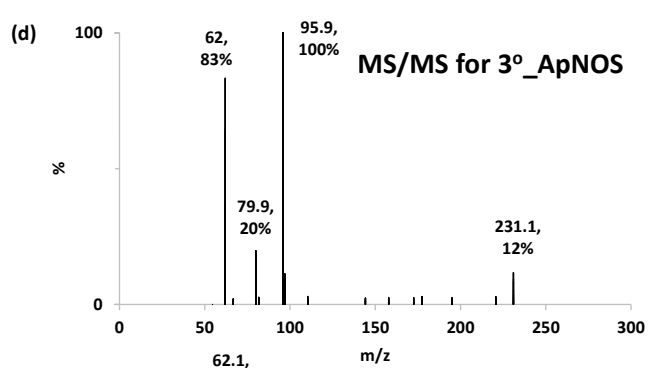

(f)
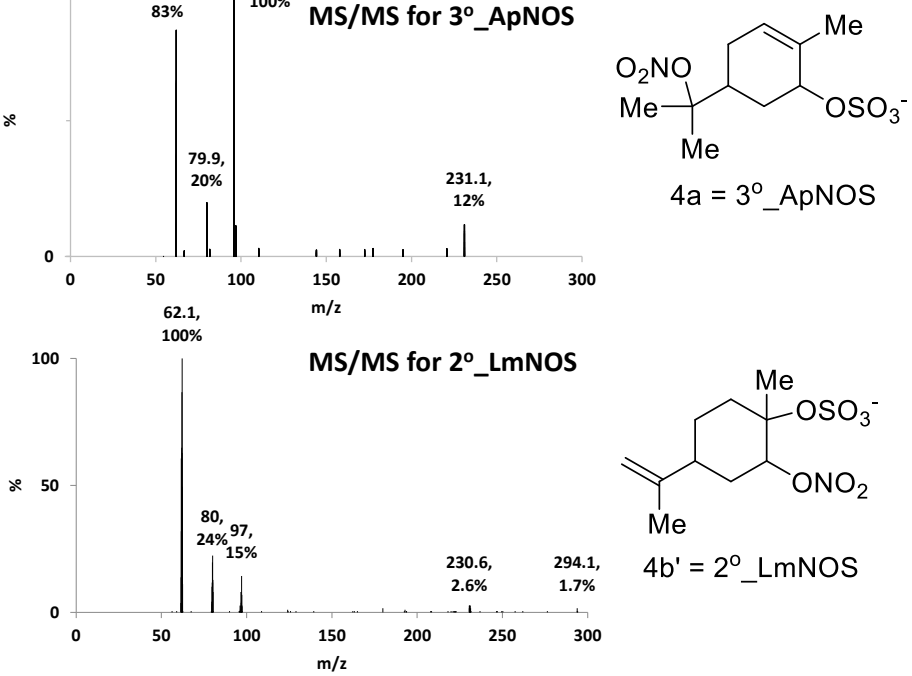

$4 a=3^{\circ}$ ApNOS

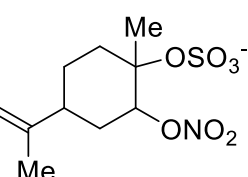

$4 b^{\prime}=2^{\circ}{ }_{-}$LmNOS $\begin{array}{ll} & 62.1, \\ \text { (h) } & 100 \%\end{array}$

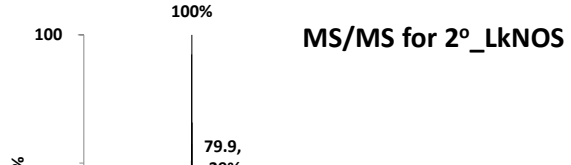

$\therefore$

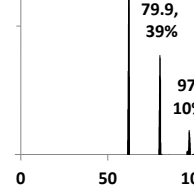

79.9,
$39 \%$

$5 b^{\prime}=2^{\circ}$ LkNOS

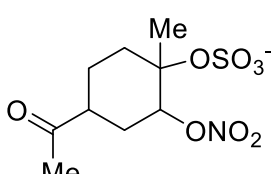<smiles>CC(=O)C1CCC(C)(O[N+](=O)[O-])C(OS(=O)(=O)O[Na])C1</smiles>

$5 b=3^{\circ}$ LkNOS

$5 b^{\prime}=2^{\circ}$ LkNOS

(k) $\quad 100 \mathrm{MS} / \mathrm{MS}$ for $2^{\circ}{ }^{\circ}$ BcpNOS ${ }^{230.7}, \quad{ }^{288.1,}$

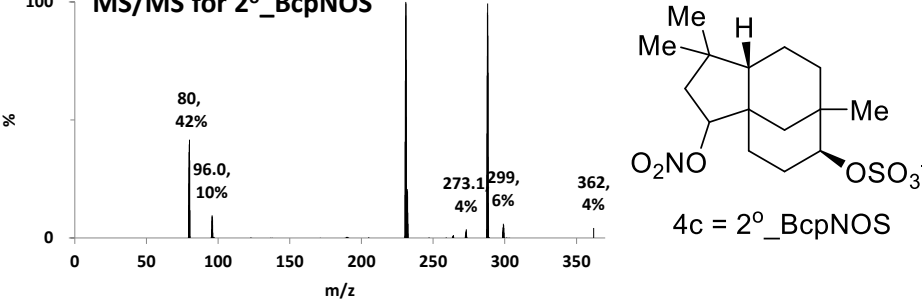


Figure S19. Comparison of MS/MS spectra of NOS $_{\mathrm{TP}}$ compounds in ambient samples and in standard solutions. (a) $\mathrm{m} / z 294$ (MW 295) at RT $=16.61 \mathrm{~min}$ in the ambient sample, (b) $\mathrm{m} / \mathrm{z} 294$ (MW 295) at RT $=16.61 \mathrm{~min}$ for the $2^{\circ}{ }_{-}$ApNOS standard, (c) $\mathrm{m} / \mathrm{z} 294$ (MW 295) at RT $=18.44 \mathrm{~min}$ in the ambient sample, (d) $m / z 294$ (MW 295) at RT $=18.44$ min for the $3^{\circ}{ }_{-}$ApNOS standard, (e) $m / z 294$ (MW 295) at RT $=$ $20.45 \mathrm{~min}$ in the ambient sample, (f) $\mathrm{m} / z 294$ (MW 295) at RT $=20.45 \mathrm{~min}$ for the $2^{\circ}{ }_{-}$LmNOS standard, (g) $\mathrm{m} / z 296$ (MW 297) at RT $=13.44 \mathrm{~min}$ in the ambient sample, (h) $\mathrm{m} / z 296$ (MW 297) at RT $=13.44$ min for the $2^{\circ}{ }_{-}$LkNOS standard, (i) $\mathrm{m} / z 296$ (MW 297) at RT $=13.44 \mathrm{~min}$ for the $3^{\circ} \& 2^{\circ}{ }_{-} \mathrm{LkNOS}$ standard isomers, (j) $\mathrm{m} / z 362$ (MW 363) at RT $=23.57 \mathrm{~min}$ in the an ambient sample, (k) $\mathrm{m} / z 362$ (MW 363 ) at RT $=23.57$ min for the $2^{\circ}{ }_{-}$BcpNOS standard. Note that the relative intensities of the products ions in ambient samples may exhibit notable variations due to the generally low concentrations encountered, in comparison with those of the authentic standards. The presence of characteristic product ions helps confirm identification of an NOS compound, however, the quantitative information of the relative intensity should not be regarded as a reliable identification criterion. 


\section{S6. Additional Supplementary plots}

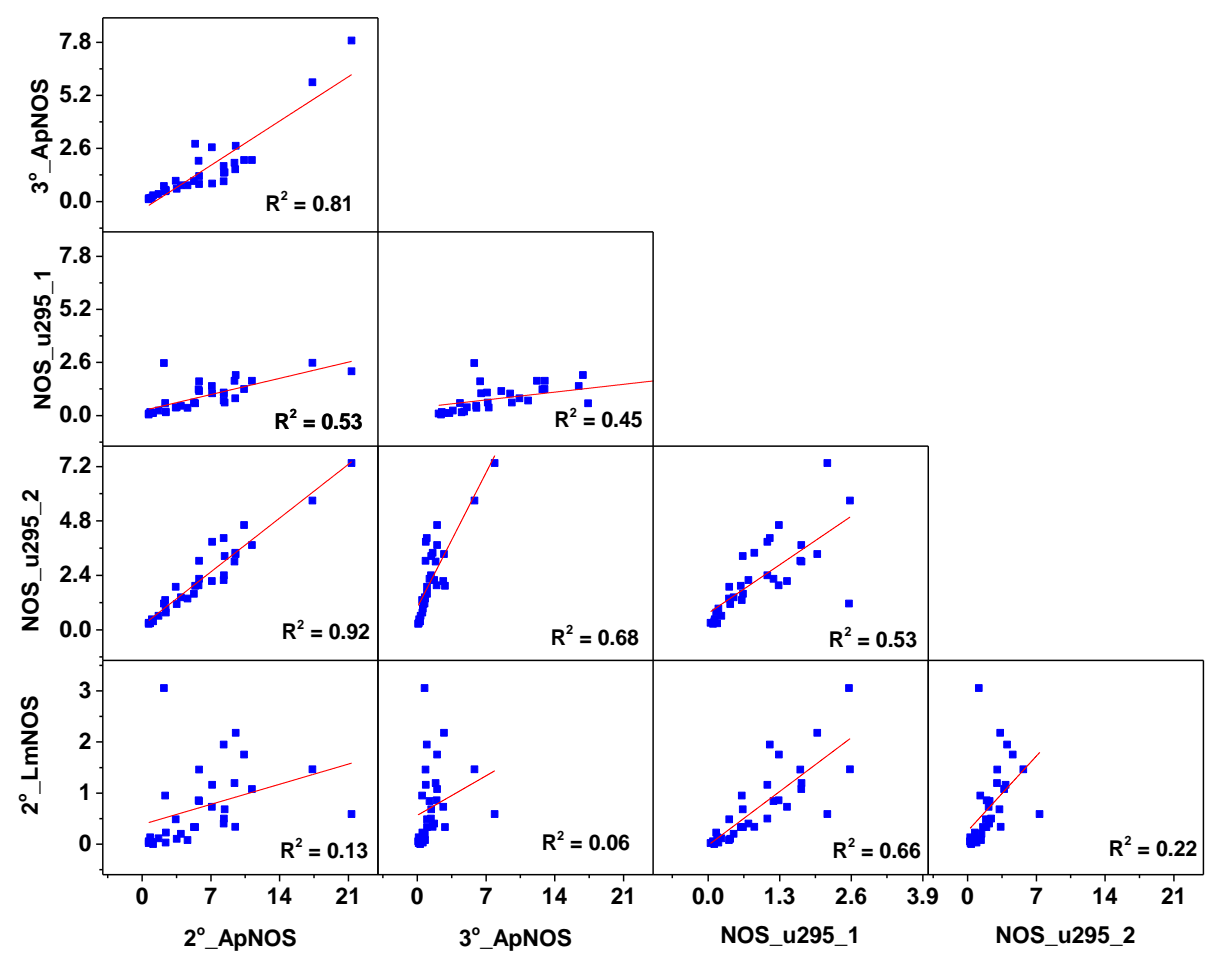

Figure S20. Correlation plots of MW295 peaks in the ambient data

(a)

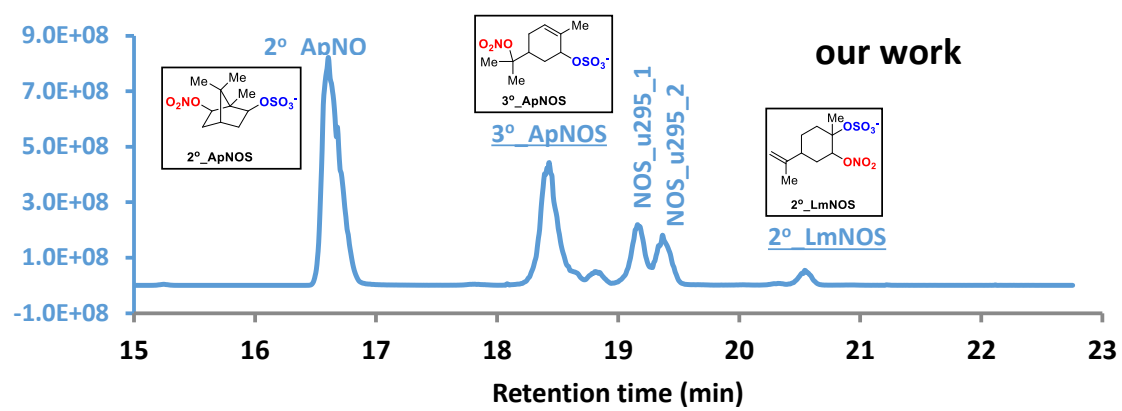

(b)

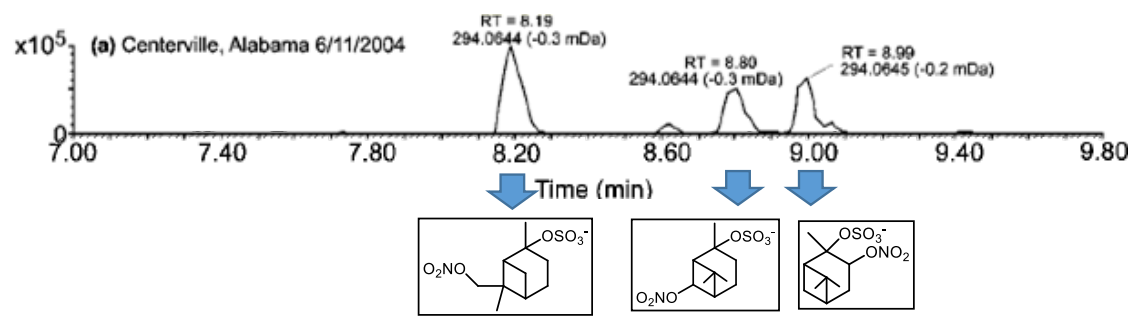

Figure S21. Comparison of extracted ion chromatograms for the MW295 NOSs between this work and the work by Surratt et al (2008). (a) Extracted ion chromatogram using Orbitrap MS for $\mathrm{m} / z$ 294.06530 (MW 295) from a Shanghai sample collected on Feb. 25, 2017. (b) Extracted ion chromatogram for MW 295 peaks in an Alabama sample (Surratt et al., 2008). The proposed MW295 NOSs are also labelled in the chromatogram. 


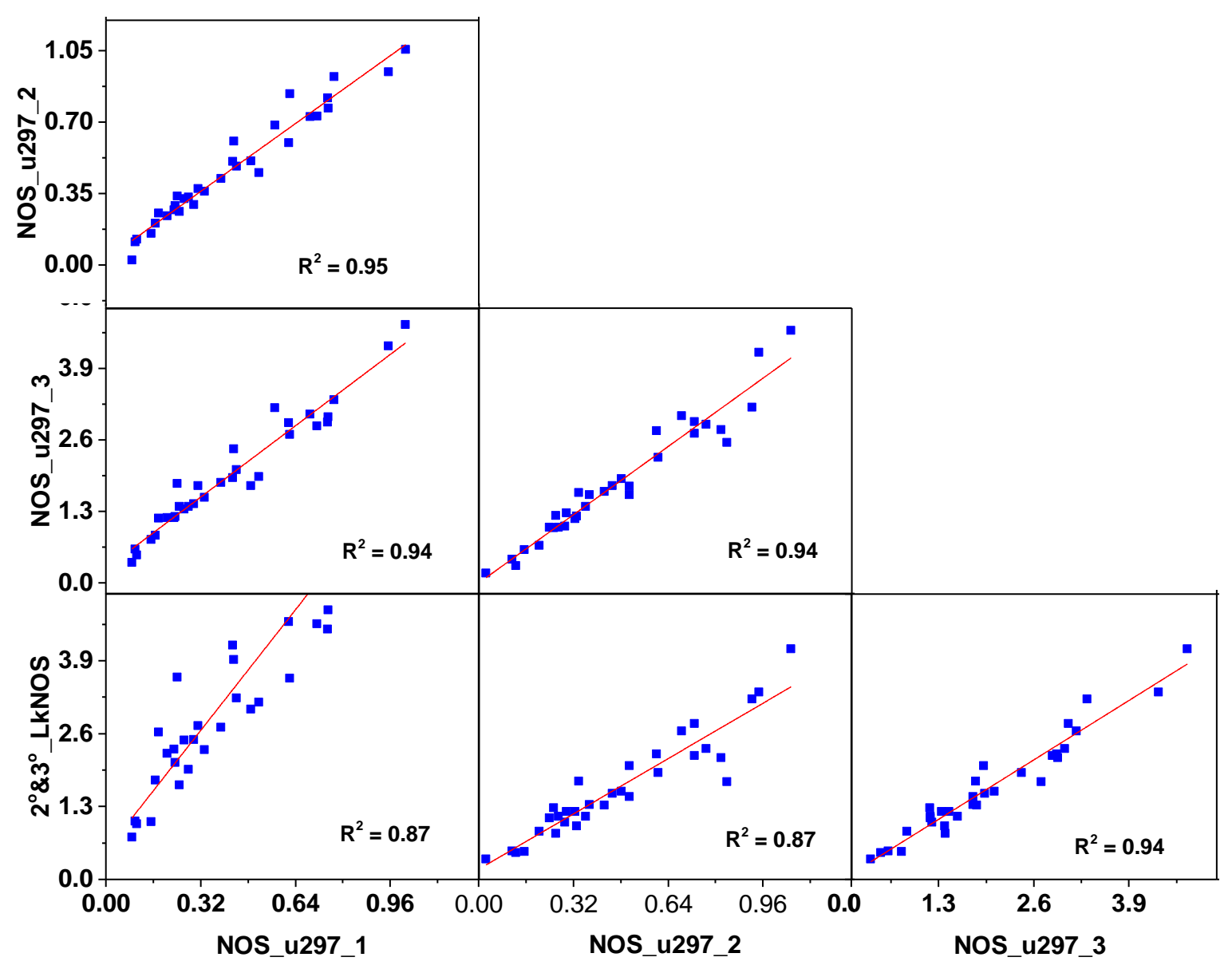

Figure S22. Correlation plots of MW297 peaks in the ambient data 


\section{(A) Our work}

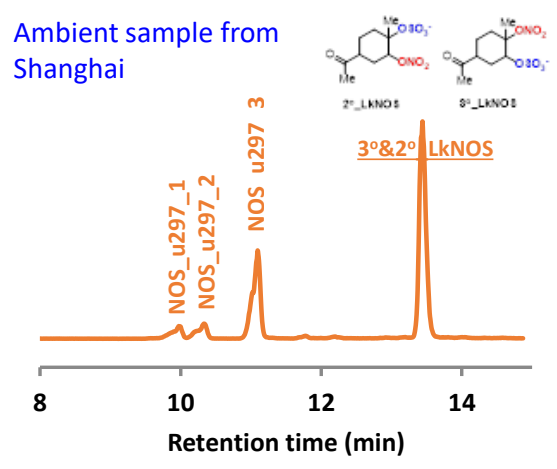

(C) Fig. 6 in Surrratt et al 2008

d-limonene $/ \mathrm{H}_{2} \mathrm{O}_{2} / \mathrm{NO} /$ acidic seed chamber experiment

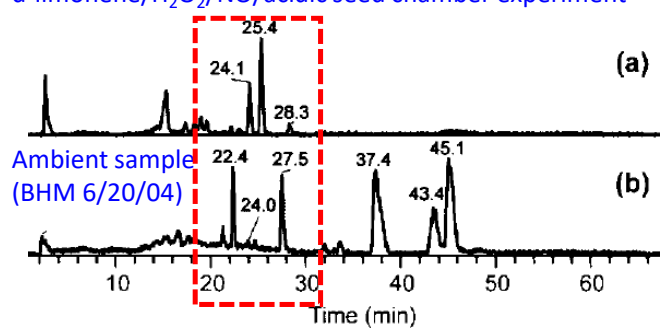

Top chromatogram: Peaks at RT 24.1, 25.4, \& 28.3 min were proposed to correspond to structures 1,2 and 3 in panel (D).

Bottom chromatogram: the middle peak at RT 24.0 min corresponded to the second eluting MW296 peak from the chamber samples. The peaks at 22.4 and 27.5 min were suggested to derive from other monoterpenes such as myrcene, ocimene).

(B) Fig. 3s in Surrratt et al 2008

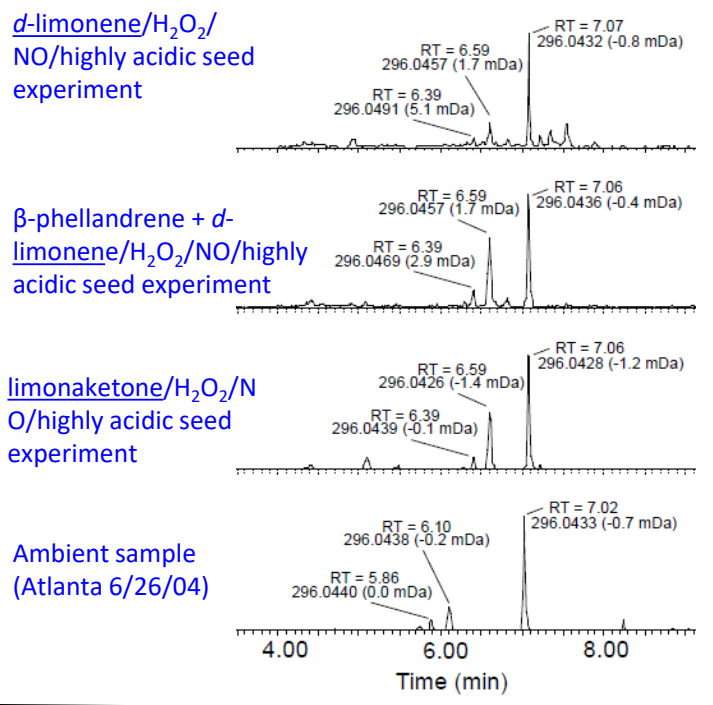

(D) MW297 NOS structures proposed in Surrratt et al 2008<smiles>CC(=O)C1CC[C@](C)(O)C(O)(O)O1</smiles>

1 and 2 (297)<smiles>CC(=O)C1CC[C@](C)(O[Na])C[C@H]1O[Na]</smiles>

$3(297)$

Figure S23. Comparison of extracted ion chromatograms for the MW297 NOSs between this work and the work by Surratt et al (2008). (a) Extracted ion chromatogram using Orbitrap MS for $m / z 296.04456$ from a Shanghai sample collected on Feb. 25, 2017. (b) and (c) Extracted ion chromatograms for MW 297 peaks for chamber experiments and ambient samples (partial duplication of Figure 3s and Figure 6 in Surratt et al. (2008)). (d) Proposed MW297 NOS structures by Surratt et al (2018). 


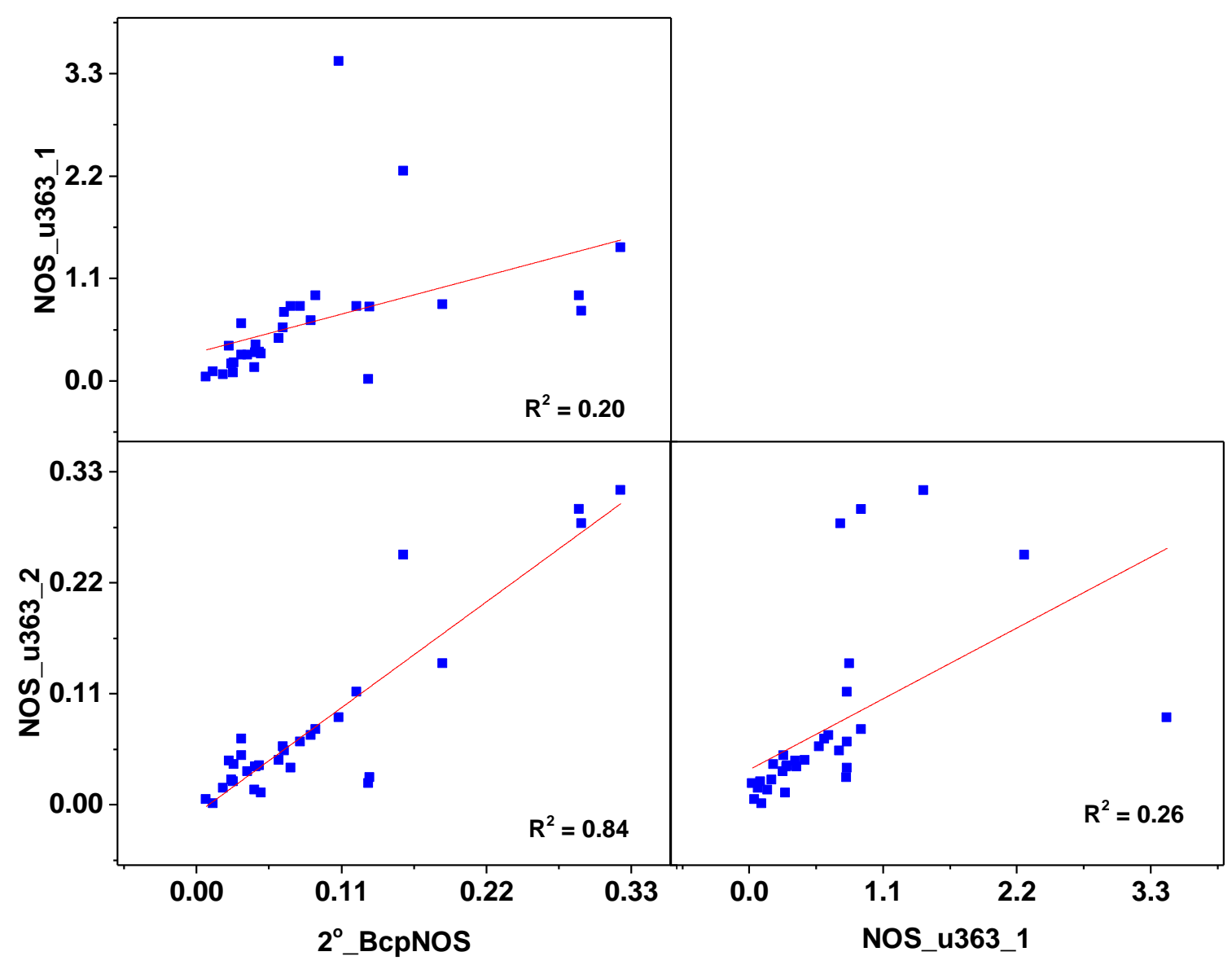

Figure S24. Correlation plots of MW363 peaks in the ambient data 